\title{
Protein Structure Refinement Algorithms
}

\author{
Thesis by \\ Mohsen Chitsaz \\ In Partial Fulfillment of the Requirements for the \\ degree of \\ $\mathrm{PhD}$ in Molecular Biophysics and Minor in Computer \\ Science
CALIFORNIA INSTITUTE OF TECHNOLOGY
Pasadena, California
2014

(Defended March 14, 2014) 


\section{Acknowledgements}

I would like to express my special appreciation and thanks to my advisor Professor Dr. Stephen L. Mayo, for being a tremendous mentor for me. I would like to thank you for encouraging my research and for allowing me to grow as a research scientist. Your advice on both research as well as on my career have been priceless. I will always look back and appreciate the chance that I was given to have you as my mentor. I would also like to thank Professor William A. Goddard, Professor Pamela J. Bjorkman, and Professor Chris Umans for serving as my committee members. I would also like to thank Dr. Marie Arie for her help with writing manuscripts and her comments on the organization of the text of this thesis. I also would like to thank Rhonda DiGuisto and everyone else in Mayo Lab. I also would like to express my great appreciation and thanks to the graduate office, Dean Joseph Shepherd and Ms. Natalie Gilmore for their invaluable kindness and help with the students in tough times. I would also like to thank Daniel Yoder and ISP for their great help throughout my programs.

Words cannot express how grateful I am to my parents for all of the sacrifices that they have made to provide me with the opportunity to be able to focus on developing myself scientifically and professionally, and explore and investigate my research ideas, and pursue my education. Your prayers and inspirations for me was what made me succeed thus far. I would also like to thank my siblings and my family for all their support, help, inspirations and encouragements. I also would like to greatly thank all my friends: Alireza Mohammad Karim, Bernardo Sosa Padilla Araujo, Alborz Mahdavi, Yelena Tuzova, Niema Pahlevan, Shervin Taghavi, Aydin 
Babakhani, Masoud Farivar, Asghar Aryanfar, Vanessa Heckman, and Gokjan Karakus all of whom have made my residence at Caltech very memorable, enjoyable and pleasant. 


\begin{abstract}
Protein structure prediction has remained a major challenge in structural biology for more than half a century. Accelerated and cost efficient sequencing technologies have allowed researchers to sequence new organisms and discover new protein sequences. Novel protein structure prediction technologies will allow researchers to study the structure of proteins and to determine their roles in the underlying biology processes and develop novel therapeutics.

Difficulty of the problem stems from two folds: (a) describing the energy landscape that corresponds to the protein structure, commonly referred to as force field problem; and (b) sampling of the energy landscape, trying to find the lowest energy configuration that is hypothesized to be the native state of the structure in solution. The two problems are interweaved and they have to be solved simultaneously. This thesis is composed of three major contributions. In the first chapter we describe a novel high-resolution protein structure refinement algorithm called GRID. In the second chapter we present REMC GRID, an algorithm for generation of low energy decoy sets. In the third chapter, we present a machine learning approach to ranking decoys by incorporating coarse-grain features of protein structures.
\end{abstract}




\section{Figures and Tables}

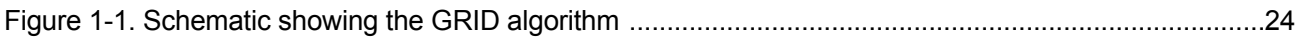

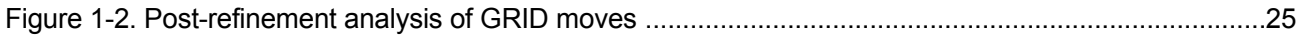

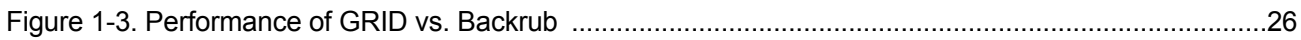

Figure 1-4. Backbone RMSD with respect to starting crystal structure ....................................................27

Figure 1-S1. Energy improvement following refinement with GRID ...................................................28

Figure 1-S2. GRID moves plotted as a function of residue number ..............................................29-31

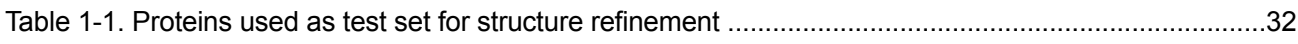

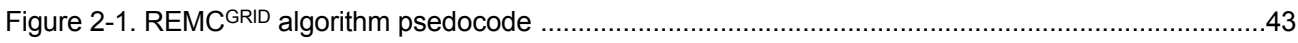

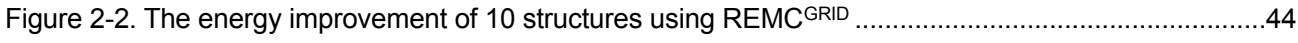

Figure 2-3. REMCGRID energy improvement compared to GRID ….....................................................45

Figure 2-4. The ratio of energy improvement of REMCGRID vs. GRID ....................................................46

Figure 2-5. Computational effort comparison of REMCGRID and GRID .................................................47

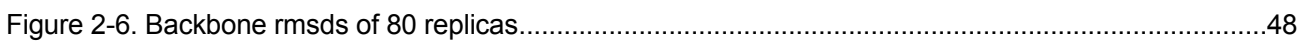

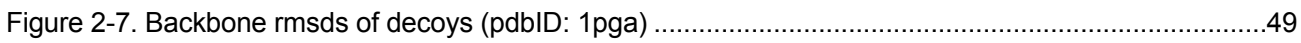

Figure 2-8. Backbone rmsd vs. energy of decoys generated .........................................................50

Figure 2-9. Backbone rmsd distribution of decoy sets generated by REMC ${ }^{\text {GRID }}$....................................51

Figure 2-10. Comparison of Rosetta decoys and REMC GRID ........................................................52

Table 2-1. Ten high-resolution structures used in testing REMC GRID ................................................53

Figure 3-1. Coarse-grained model of protein structures …...................................................................62

Figure 3-2(A,B,C). Energy model for the coarse-grained model proposed in Chapter $3 \ldots \ldots \ldots \ldots \ldots \ldots \ldots \ldots . . . . . . . . . . . .63$

Figure 3-3. Pairwise energies among amino acid groups...................................................................66

Figure 3-4. Backbone rmsd distribution of decoys generated by REMC GRID ...........................................67

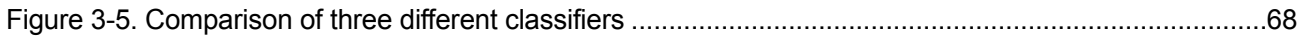

Figure 3-S1. Coarse-grained score vs. backbone RMSD (pdbID: 1fkb) …........................................69

Figure 3-S2. Comparison of coarse-grained score vs. minimum and average rmsd ...............................70

Figure 3-S3. Protein structure description in the coarse-grained model ...............................................71

Figure 3-S4. Modeling the scoring function using sigmoid functions ......................................................72

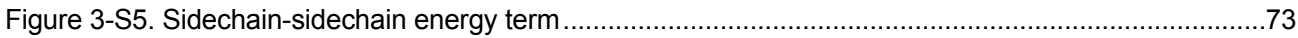

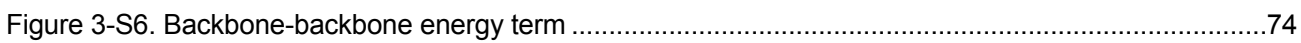

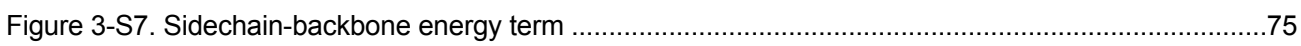

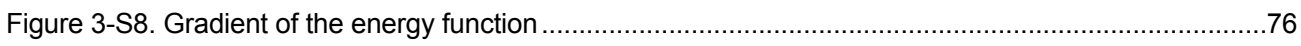

Figure 3-S9. Coarse-grained model, energy function, and the gradient formulation.................................77

Figure 3-S10. Size distribution of protein structures used in decoy generation .....................................78 


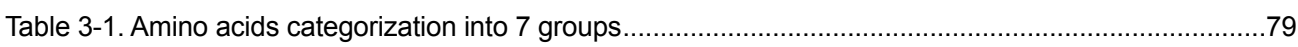

Table 3-2. Pairwise energies that are used in the coarse-grained scoring function ..................................80

Table 3-3. Comparison of three different classifiers trained on decoys .................................................. 81 


\section{Nomenclature}

RMSD: Root Mean Square Deviation

GRID: A high-resolution refinement algorithm

REMCGRID: Replica Exchange Monte Carlo GRID

AU: Arbitrary Units

SVM: Support Vector Machine

PDB: Protein Data Bank

Residue: an amino acid in the context of a protein structure

ф: Dihedral angle formed on the backbone of a residue by C, N, C-alpha, C atoms

$\Psi:$ Dihedral angle formed on the backbone of a residue by $\mathrm{N}, \mathrm{C}$-alpha, $\mathrm{C}, \mathrm{N}$ atoms

Side-chain: Chemical group attached to the backbone of the protein, determining the type of amino acid

Conformation: 3-dimensional state of a protein structure in Cartesian space

Forcefield: a scoring function that takes a conformation of a protein and assigns energy to it

Decoy: a conformation of a protein, typically, perturbed state of a protein structure 


\section{TABLE OF CONTENTS}

Acknowledgements ..............................................................................

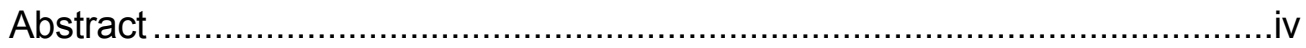

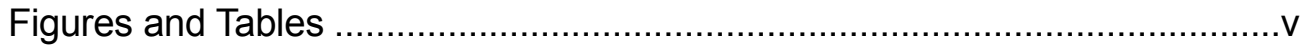

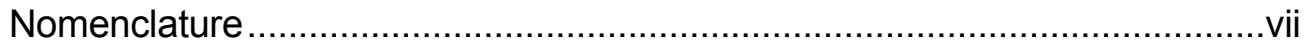

Table of Contents ....................................................................................ii

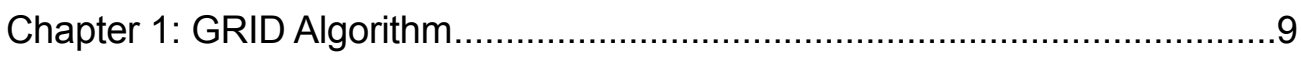

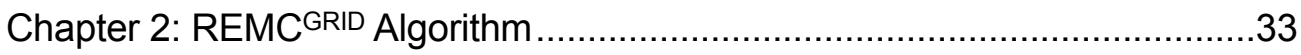

Chapter 3: Coarse-grained Scoring and Machine Learning ........................54

Appendix A: Header file for the algorithms .................................................83

Appendix B: Main code for the algorithms ......................................... 88 


\section{Chapter 1}

\section{GRID: A High-Resolution Protein Structure Refinement Algorithm}

The text of this chapter is adapted from a published review article that was co-authored with Professor Stephen L. Mayo

Chitsaz M, Mayo SL., J Comput Chem 2013; 34:445-450. 


\section{Abstract}

The energy-based refinement of protein structures generated by fold prediction algorithms to atomic-level accuracy remains a major challenge in structural biology. Energybased refinement is mainly dependent on two components: (1) sufficiently accurate force fields, and (2) efficient conformational space search algorithms. Focusing on the latter, we developed a high-resolution refinement algorithm called GRID. It takes a three-dimensional protein structure as input and, employing an all-atom force field, attempts to improve the energy of the structure by systematically perturbing backbone dihedrals and side chain rotamer conformations. We compare GRID to Backrub, a stochastic algorithm that has been shown to predict a significant fraction of the conformational changes that occur with point mutations. We applied GRID and Backrub to 10 high-resolution $(\leq 2.8 \AA)$ crystal structures from the Protein Data Bank and measured the energy improvements obtained and the computation times required to achieve them. GRID resulted in energy improvements that were significantly better than those attained by Backrub while expending about the same amount of computational resources. GRID resulted in relaxed structures that had slightly higher backbone RMSDs compared to Backrub relative to the starting crystal structures. The average RMSD was $0.25 \pm 0.02 \AA$ for GRID versus $0.14 \pm 0.04$ $\AA$ for Backrub. These relatively minor deviations indicate that both algorithms generate structures that retain their original topologies, as expected given the nature of the algorithms. 
Key words: protein structure refinement; flexible backbone; energy-based refinement; conformational search; backrub motion 


\section{Introduction}

Although much progress has been made in recent years, the ability to predict the threedimensional structure of a protein from its amino acid sequence remains a major challenge in structural biology. Good prediction techniques would allow us to obtain the structures of novel protein sequences without having to determine them experimentally and would be particularly useful for membrane and other proteins whose structures are experimentally difficult to obtain or are highly diverged from the set of proteins with existing structures ${ }^{1}$. The knowledge obtained from accurate protein structure prediction could improve our understanding of how proteins function, aid in the discovery of proteins with new and improved properties, and facilitate the development of new drugs ${ }^{2,3}$. Many biological applications, including studies on enzymatic activity, virtual ligand screening, structure-based protein function prediction, and structure-based drug design require knowledge of protein structures at atomic resolution ${ }^{1}$. In addition, the expansion of highthroughput genome sequencing projects has led to a continually widening gap between protein sequence data and the number of solved structures. Thus, there is a significant need for computational techniques that can model protein structures with atomic accuracy comparable to experimental methods.

Protein structure prediction methods can be broadly classified into three main categories: comparative modeling, threading, and template-free modeling ${ }^{4}$. In comparative modeling, the protein structure is built by using sequence alignments to find the best matching evolutionarily related protein that has a solved structure (the template) ${ }^{4}$. Most structures constructed using this approach have a root mean square deviation (RMSD) of 1-2 $\AA$ from the experimental structure ${ }^{5}$. In the absence of any evolutionarily related pro- 
teins, threading can be used, which matches the target protein directly to the sequences of solved three-dimensional structures. Threading methods often identify correct templates and provide models with an RMSD of 2-6 A, with inaccuracies occurring mainly in the loop regions ${ }^{6}$. For targets that have no structurally-related solved proteins $(<30 \%$ sequence similarity), template-free modeling methods are used; that is, the model is built from scratch ${ }^{5,7}$. Successful prediction using template-free modeling is limited to small proteins $\left(<120\right.$ residues), with accuracy in the range of $4-8 \AA^{8}$. Much effort has been expended in the last several decades to achieve the current levels of success in each of these three categories. However, the resulting models are still low-resolution and further refinement is needed in order to achieve the atomic-level accuracy required for most biological applications. Refinement of low-resolution structures to higher resolution models therefore remains a major challenge in the field of protein structure prediction ${ }^{1,}$, 9-11. Recognition of the importance of refinement is reflected by the addition of a refinement category in the eighth Critical Assessment of Techniques for Protein Structure Prediction $(\mathrm{CASP} 8)^{12}$.

Approaches to high-resolution refinement have included molecular mechanics energy minimization and Monte Carlo methods including replica exchange Monte Carlo1, 13-16. Knowledge-based statistically derived potentials have also been incorporated into these techniques ${ }^{17}$. Recently, a flexible backbone modeling algorithm was applied ${ }^{18-20}$. All these methods have attained some degree of success; however, none has emerged as clearly superior ${ }^{13,14,16}$. Current methods require improvements in terms of computational efficiency and/or accuracy ${ }^{18,20-23}$. For those approaches that generate an ensemble containing a large number of low-resolution structures, refinement algorithms must be very effi- 
cient in processing many structures in a reasonable amount of time. In addition, the refined structure must accurately reflect the naturally folded state. Because the minimum energy configuration is considered to be the best estimate of native structure ${ }^{24,25}$, highresolution refinement methods must be able to efficiently find lower energy configurations. The energy improvement attained relative to the starting structure is a measure of a method's progress towards a model consistent with the experimental data, assuming an accurate energy function. Thus, refinement procedures typically employ multiple refinement cycles in which the energy of a low-resolution structure is iteratively reduced. The energy of the structure is calculated based on molecular mechanics force fields that describe the underlying physics and chemistry of protein molecules ${ }^{14,16}$. Knowledge-based force fields are also commonly used ${ }^{26}$.

To address the problems of computational efficiency and accuracy, we devised a highresolution refinement algorithm called GRID. The method sequentially applies perturbations to a structure's $\varphi$ and $\psi$ angles and side chain rotamers, accepting perturbations that produce the most improvement in the energy of the structure. Unlike many refinement methods that rely on stochastic techniques, GRID has been tuned to use a single pass search strategy and therefore requires less computational effort. We compared GRID to Backrub ${ }^{27}$, a flexible backbone modeling method that is able to predict most of the small conformational changes associated with point mutations ${ }^{18,20}$. GRID and Backrub were applied to a set of 10 high-resolution crystal structures from the PDB, and the algorithms were assessed in terms of energy improvement and computational efficiency. 


\section{Computational Methods}

\section{GRID refinement algorithm}

GRID is a refinement method that takes a three-dimensional protein structure as input and, employing an all-atom force field, attempts to improve the energy of the structure using a move set that allows for backbone and side chain flexibility (Fig. 1). Three degrees of freedom are considered: $\varphi$ and $\psi$ angle perturbations and side-chain rotamer optimization. The algorithm starts from the first residue ( $\mathrm{N}$ terminus) and evaluates all combinations of $\Delta \varphi, \Delta \psi$, and rotamer, given a specified maximum perturbation angle and precision level (perturbation angle step size). $\Delta \varphi$ and $\Delta \psi$ perturbations are applied to the C-terminal protein segment. The continuous space of $\Delta \varphi, \Delta \psi$ perturbations is broken down into discrete grid points around the current $\varphi$ and $\psi$ angles of the residue. Similarly, a rotamer library is used to define a discrete set of rotamers for the residue. For each combination of $\Delta \varphi, \Delta \psi$, and rotamer available for a given residue, the algorithm perturbs the structure and calculates the energy of the trial configuration (the rest of the molecule is treated as a rigid body). This information is used to fill out a three-dimensional matrix that reflects the discretized energy space for the residue. The move that results in the lowest energy is then applied to the structure before proceeding to the next residue. GRID marches forward from $\mathrm{N}$ to $\mathrm{C}$ terminus, sequentially calculating the energy matrix and applying the lowest energy move on each of the residues. When the last residue is reached, the algorithm can terminate or return to the $\mathrm{N}$ terminus for a subsequent iteration.

Note that although the moves are restricted to local perturbations relative to a single residue, the perturbation selected influences the entire structure. Thus, relatively minor local changes can propagate down the chain resulting in larger shifts in the conformation 
of the protein. In order to further improve the energies, at the end of the procedure, the algorithm walks through the structure once (from $\mathrm{N}$ to $\mathrm{C}$ terminus) and performs four steps of local conjugate gradient energy minimization on each residue while keeping the rest of the structure fixed in Cartesian space.

\section{GRID implementation and parameterization}

The GRID refinement algorithm was developed in $\mathrm{C}++$ and incorporated into the Triad protein design software package ${ }^{33}$. The Rosetta force field with default parameters ${ }^{28}$ was used to calculate the energy of the protein conformations. Rotamers were selected from a backbone-dependent side-chain rotamer library ${ }^{29}$.

As noted above, GRID takes as input four main parameters: (1) the maximum perturbation angle $(\Delta \varphi, \Delta \psi)$ for each iteration, (2) the precision level (step size for $\Delta \varphi$ and $\Delta \psi$ ) for each iteration, (3) the rotamer library used for each iteration, and (4) the number of iterations. GRID was run on 2-10 test proteins using several combinations of these parameters to determine the optimal protocol for refinement. Values for maximum $\Delta \varphi$ and $\Delta \psi$ ranged from $\pm 0.1^{\circ}$ to $\pm 8.0^{\circ}$ and precision levels ranged from $0.1^{\circ}$ to $8.0^{\circ}$, forming $\Delta \varphi \times \Delta \psi$ matrices containing from 9 to 441 grid points; the number of iterations ranged from 1 to 10. The same set of rotamers (from the Dunbrack backbone-dependent rotamer library ${ }^{29}$ ) was used in every case.

\section{Backrub algorithm}

The Backrub concept was developed by the Richardson lab ${ }^{27}$ and was implemented by the Kortemme ${ }^{18,20,30}$ and Donald ${ }^{31}$ groups. Its move set was derived from the observation 
that ultra-high-resolution crystal structures often show alternate conformations in which subtle local backbone motion is coupled to much larger two-state changes in side-chain conformation ${ }^{18,20,21}$. This "backrub" motion supplies a common type of local plasticity in protein backbones. A refinement algorithm based on the Backrub concept performs 10,000 steps of Monte Carlo simulation using Metropolis acceptance criteria. In every step, Backrub employs two types of moves with equal probability: (1) it replaces a sidechain rotamer (randomly chosen from a specified rotamer library) at a randomly chosen residue position, or (2) it performs a Backrub move in which a randomly chosen 2- or 3residue fragment is rotated about the axis defined by the $\mathrm{C}_{\alpha}$ atoms of the first and the last residue of the fragment. The bond angles extending from the $C_{\alpha}$ atoms of the first and the last residues are then optimized using a bond angle potential. We used the Rosetta version 3.1 implementation of Backrub, with parameters as described in Friedland et al ${ }^{18}$.

\section{Test sets and assessment criteria}

GRID and Backrub were initially tested on 10 single-chain high-resolution $(\leq 2.8 \AA)$ crystal structures from the PDB representing proteins of various sizes and folds $(\alpha, \beta, \alpha+\beta$, and $\alpha / \beta)$ (Table 1). Two criteria were used to compare the algorithms: (1) energy improvement (relative to that of the starting structure), and (2) computation time. The validity of the first criterion is based on the assumption that the minimum energy configuration is the best estimate of the native state 24,32 and that the degree of energy improvement attained reflects the method's success in moving toward the native form and, perhaps more importantly, allowing selection of the best structural models from an ensemble of predicted structures. 


\section{Results}

GRID parameterization: smaller perturbations of $\varphi$ and $\psi$ allow more global structure refinement

Initial trials on $2-10$ test proteins indicated that refinements done using a single iteration and the lowest precision level, a $3 \times 3 \Delta \varphi \times \Delta \psi$ matrix, produced energy improvements that were at least $70 \%$ of the values obtained with larger matrices $(11 \times 11)$ and multiple iterations (up to 10). These initial studies also showed that perturbations of $\varphi$ and $\psi$ greater than $\pm 2.0^{\circ}$ caused no significant energy improvement over GRID refinements performed using smaller perturbations. More extensive tests were performed using smaller perturbation angles that ranged from $\pm 0.1^{\circ}$ to $\pm 1.9^{\circ}$, and all of these resulted in the same level of energy improvement (Fig. S1). Completely eliminating $\varphi$ and $\psi$ perturbations (i.e., sidechain rotamer optimization alone) was less effective at energy improvement (Fig. S2). We then conducted a post-refinement analysis of the moves that had been applied to achieve the final refined structures (Fig. 2). The moves were divided into four types that differ in their effects on protein structure: type $A$, the backbone is not perturbed $(\Delta \varphi=0, \Delta \psi=0)$; type $B$, only one of the dihedrals is perturbed $(\Delta \varphi=0, \Delta \psi \neq 0)$ or $(\Delta \varphi \neq 0, \Delta \psi=0)$; type $C$, both dihedrals are perturbed by the same amount in the same direction $(\Delta \varphi=\Delta \psi)$; and type $D$, both dihedrals are perturbed by the same amount, but in opposite directions $(\Delta \varphi=-\Delta \psi)$. Side chain rotamer optimization was allowed in all cases. The frequency of occurrence of each of these different types of moves was then calculated. As seen in Figure 2, move type A (no backbone perturbation) became increasingly dominant at larger perturbation angles, representing up to $85 \%$ of all the moves ap- 
plied. The next most common move was type B, followed by type $D$, then type $C$; this trend was consistent at all perturbation angles tested. At the smallest perturbation angle $\left(0.1^{\circ}\right)$, the type A move (no backbone perturbation) was no longer dominant and occurred at about the same frequency as types B and D ( 27\%).

In choosing the parameters for refinement with GRID, one of the criteria was that the perturbations be fairly evenly dispersed throughout the protein structure and not restricted to specific regions. We found that using the smallest perturbation $\left( \pm 0.1^{\circ}\right)$ for $\Delta \varphi$ and $\Delta \psi$ gave the best results (i.e., the frequency of dihedral angle change was similar for all residues), whereas with larger perturbations, most of the moves caused no backbone movement (only the rotamer was changed), and moves that did perturb the backbone were restricted to residues near the termini (Fig. S3). These results led us to choose a maximum perturbation angle of $\pm 0.1^{\circ}$. To minimize computation time, the lowest values were also used for the number of iterations and for the precision level. The final GRID refinement parameters are therefore as follows: maximum perturbation angle $(\Delta \varphi$, $\Delta \psi)= \pm 0.1^{\circ}$, precision level $=0.1^{\circ}$, and iterations $=1$, with rotamers from the Dunbrack backbone-dependent rotamer library ${ }^{29}$.

\section{GRID outperforms Backrub}

As shown in Figure 3a, refinement with GRID resulted in structures with energy improvements ranging from $\sim 100$ to $\sim 600 \mathrm{kcal} / \mathrm{mol}$. In every case, these values were significantly larger than those obtained with a single trajectory of Backrub. Increasing the number of Backrub trajectories to 10,100 , or 1000 caused minimal additional improvement. The four steps of local conjugate gradient minimization that is applied at the end of the GRID pro- 
cedure has a significant impact on the resulting energies, increasing the overall energy improvement by about a factor of 2 (Fig. S4). The computation time expended by GRID was comparable to that required for one trajectory of Backrub (Fig. 3b); run times ranged from $45 \mathrm{sec}$ to about 6 minutes, averaging $\sim 3$ minutes on a single CPU core (Intel Xeon at $2.33 \mathrm{GHz}$ ). As expected, computational effort increased linearly with the number of Backrub trajectories, with 1000 trajectories taking $\sim 2400$ minutes for the largest protein in the test set (155 residues).

Plots of energy improvement versus protein size show an enhanced effect (greater improvement) as protein size increases; this trend is seen for both algorithms, but is more pronounced for GRID (Fig. 3c). As expected, computation time scales linearly with the number of residues for both Backrub and GRID (Fig. 3d). Taken together, these results demonstrate that GRID outperforms Backrub for high-resolution structure refinement, achieving substantially greater energy improvements in every case while expending comparable computational effort.

Calculation of the backbone RMSD of the final refined structure with respect to the starting crystal structure for each of the ten proteins shows that GRID produces somewhat larger deviations than Backrub; the average RMSD was $0.25 \pm 0.02 \AA$ for GRID versus $0.14 \pm 0.04 \AA$ for Backrub (Fig. 4). These relatively minor deviations indicate that both algorithms generate refined structures that retain their original topologies.

An implementation of GRID that uses random residue order for applying perturbations was also run and found to produce nearly identical results to the sequential $\mathrm{N}$ terminus to C terminus version described above (Fig. S5). 


\section{Discussion}

Our results indicate that the GRID algorithm is more effective in reducing the energy of structures than the Backrub algorithm: applying the same all-atom force field, GRID consistently yields lower energy configurations. Although stochastic algorithms have been successful in refining small proteins ( $<85$ residues), refinement of larger proteins has proven to be particularly difficult ${ }^{3}$. In contrast, GRID was effective on all the proteins tested, which included structures up to 155 residues. GRID also achieves these improvements with relatively little computational resources (equivalent to one trajectory of Backrub), thus making it feasible to use on even larger proteins. This level of computational efficiency is essential for the last stage of protein structure prediction, since low-resolution prediction techniques typically produce a large number of structures that must be refined in order to select the best structural model. GRID's high performance capabilities stem from using a strategy that is straightforward, but effective.

To achieve structures that are closer to the native state, refinement methods must be able to find lower energy configurations. This can be problematic for algorithms that use local perturbations to achieve refinement, as they are more likely to fall into local minima. It is possible that GRID minimizes this problem because it applies local perturbations that are propagated down the whole protein chain. These perturbations, which are effectively more global in nature, have a higher probability of explicitly moving the structure out of a local minimum. Thus, GRID may allow for a more thorough exploration of the energy landscape, making it easier to find lower energy structures. 


\section{Acknowledgments}

This work was supported by the Defense Advanced Research Projects Agency (DARPA) and a Department of Defense National Security Science and Engineering Faculty Fellowship (S.L.M). I would like to thank Marie Ary for her comments and assistance in writing the manuscript.

\section{References}

[1] G. Chopra, C. M. Summa, M. Levitt, Proc. Natl. Acad. Sci. USA 2008, 105, 2023920244.

[2] D. Baker, A. Sali, Science 2001, 294, 93-96.

[3] P. Bradley, K. M. S. Misura, D. Baker, Science 2005, 309, 1868-1871.

[4] Y. Zhang, Curr. Opin. Struct. Biol. 2009, 19, 145-155.

[5] V. Mariani, F. Kiefer, T. Schmidt, J. Haas, T. Schwede, Proteins 2011, 79 Supp/ 10, 37-58.

[6] R. Jauch, H. C. Yeo, P. R. Kolatkar, N. D. Clarke, Proteins 2007, 69 Suppl 8, $57-67$.

[7] J. Xu, J. Peng, F. Zhao, Proteins 2009, 77 Suppl 9, 133-137.

[8] J. Kopp, L. Bordoli, J. N. Battey, F. Kiefer, T. Schwede, Proteins 2007, 69 Supp/ 8, 38-56.

[9] I. H. Park, V. Gangupomu, J. Wagner, A. Jain, N. Vaidehi, J. Phys. Chem. B 2012 , $116,2365-2375$. 
[10] O. Graña, D. Baker, R. M. MacCallum, J. Meiler, M. Punta, B. Rost, M. L. Tress, A. Valencia, Proteins 2005, 61 Suppl 7, 214-224.

[11] C. Venclovas, A. Zemla, K. Fidelis, J. Moult, Proteins 2003, 53 Supp/ 6, 585-595.

[12] J. L. MacCallum, L. Hua, M. J. Schnieders, V. S. Pande, M. P. Jacobson, K. A. Dill, Proteins 2009, 77 Suppl 9, 66-80.

[13] S. Kannan, M. Zacharias, Proteins 2007, 66, 697-706.

[14] S. Kannan, M. Zacharias, J. Struc. Biol. 2009, 166, 288-294.

[15] M. Levitt, S. Lifson, J. Mol. Biol. 1969, 46, 269-279.

[16] A. Shmygelska, M. Levitt, Proc. Natl. Acad. Sci. USA 2009, 106, 1415-1420.

[17] C. M. Summa, M. Levitt, Proc. Natl. Acad. Sci. USA 2007, 104, 3177-3182.

[18] G. D. Friedland, A. J. Linares, C. A. Smith, T. Kortemme, J. Mol. Biol. 2008, 380, 757-774.

[19] M. S. Lin, T. Head-Gordon, J. Comput. Chem. 2011, 32, 709-717.

[20] C. A. Smith, T. Kortemme, J. Mol. Biol. 2008, 380, 742-756.

[21] T. E. Creighton, Biochem. J. 1990, 270, 1-16.

[22] K. A. Dill, Biochemistry 1990, 29, 7133-7155.

[23] H. Venselaar, R. P. Joosten, B. Vroling, C. A. B. Baakman, M. L. Hekkelman, E. Krieger, G. Vriend, Eur. Biophys. J. 2010, 39, 551-563.

[24] G. I. Makhatadze, P. L. Privalov, Adv. Protein. Chem. 1995, 47, 307-425.

[25] O. B. Ptitsyn, Adv. Protein. Chem. 1995, 47, 83-229.

[26] K. T. Simons, C. Kooperberg, E. Huang, D. Baker, J. Mol. Biol. 1997, 268, 209-225. 
[27] I. W. Davis, W. B. Arendall, D. C. Richardson, J. S. Richardson, Structure 2006, $14,265-274$.

[28] C. A. Rohl, C. E. Strauss, K. M. Misura, D. Baker, Methods Enzymol. 2004, 383, 66-93.

[29] R. L. Dunbrack, F. E. Cohen, Protein Sci. 1997, 6, 1661-1681.

[30] E. L. Humphris, T. Kortemme, Structure 2008, 16, 1777-1788.

[31] I. Georgiev, D. Keedy, J. S. Richardson, D. C. Richardson, B. R. Donald, Bioinformatics 2008, 24, i196-204.

[32] C. B. Anfinsen, Science 1973, 181, 223-230.

[33] Protabit, www.protabit.com.

[34] W. Kabsch, C. Sander, Biopolymers 1983, 22, 2577-2637. 
a)

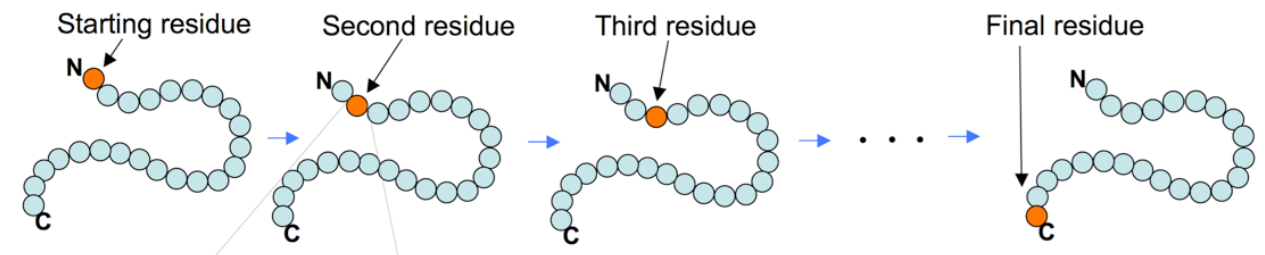

b)

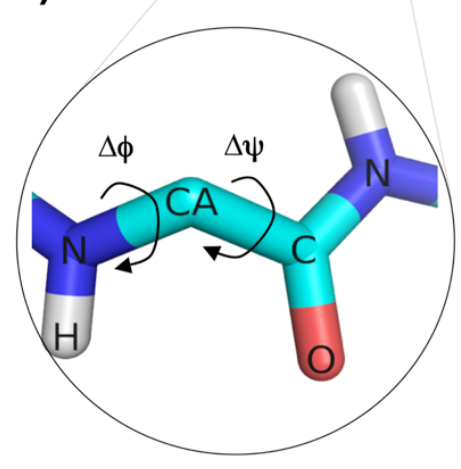

c)

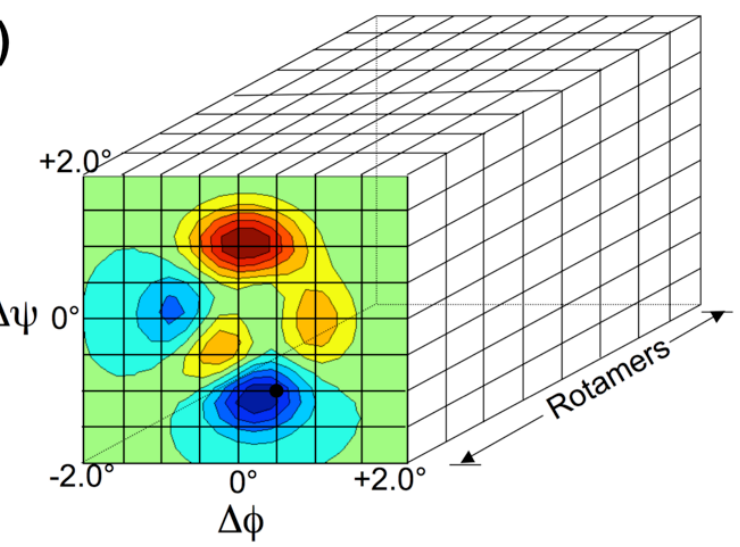

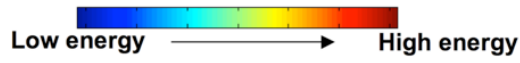

Figure 1. Schematic showing the GRID algorithm. a) In each iteration, GRID starts from the N-terminal residue and marches forward to the $C$ terminus, sequentially applying the lowest energy move on the structure before proceeding to the next residue. b) For each residue, GRID evaluates all the possible perturbations (combinations of $\Delta \varphi, \Delta \psi$, and rotamer) given a specified maximum perturbation angle and precision level, then applies the move that results in the lowest energy. c) Three-dimensional energy matrix (grid) that corresponds to all the possible perturbations $(\Delta \varphi, \Delta \psi$, and rotamer) relative to the current configuration of a residue. In this example, the maximum perturbation angle for $\Delta \varphi$ and $\Delta \psi$ is $\pm 2.0^{\circ}$, the precision level is $0.5^{\circ}$, and 8 rotamers are specified from the backbone dependent rotamer library. 


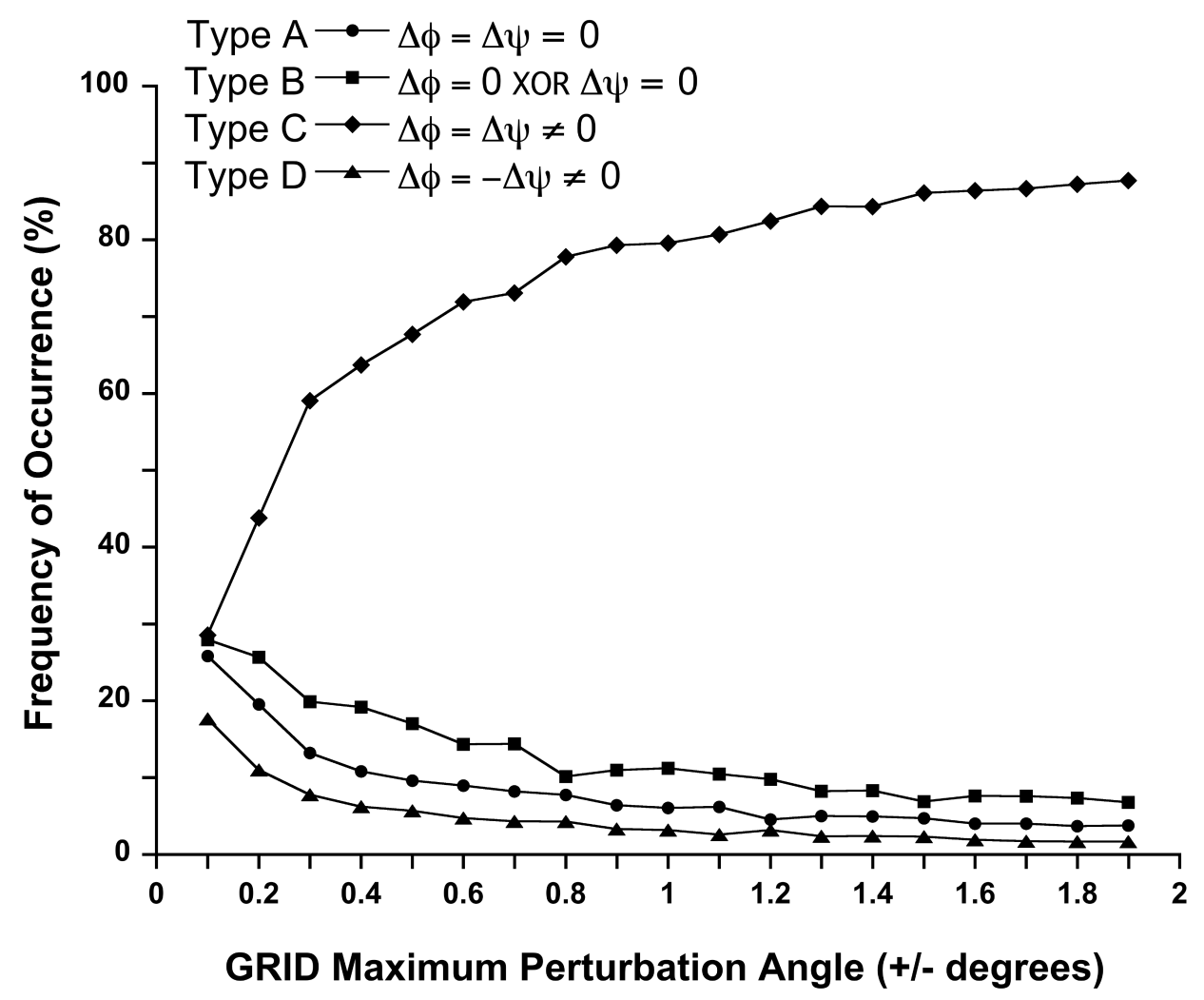

Figure 2. Post-refinement analysis of GRID moves for different maximum dihedral angle perturbations. A single iteration of GRID was run on each of the 10 test proteins using maximum perturbation angles ranging from $\pm 0.1^{\circ}$ to $\pm 1.9^{\circ}$; the precision level was always set to form the smallest $\Delta \varphi \times \Delta \psi$ matrix (9 grid points). GRID moves were divided into four types: no perturbation of the backbone (type A), perturbation of $\varphi$ or $\psi$, but not both (type B), perturbation of both $\varphi$ and $\psi$ by the same amount in the same direction (type $C$ ), and perturbation of both $\varphi$ and $\psi$ by the same amount, but in opposite directions (type $D$ ). The frequency of occurrence of each of the four different types of moves was calculated; points are the average for the 10 test proteins. 
a)

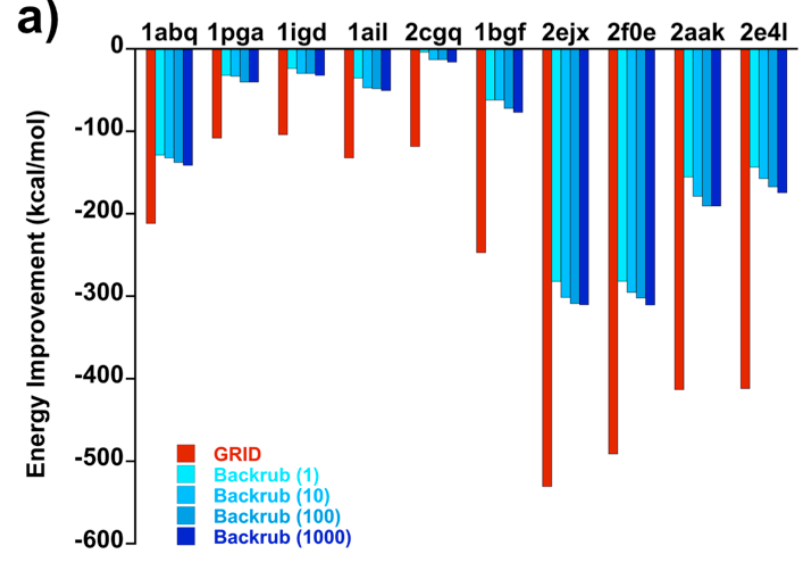

b)

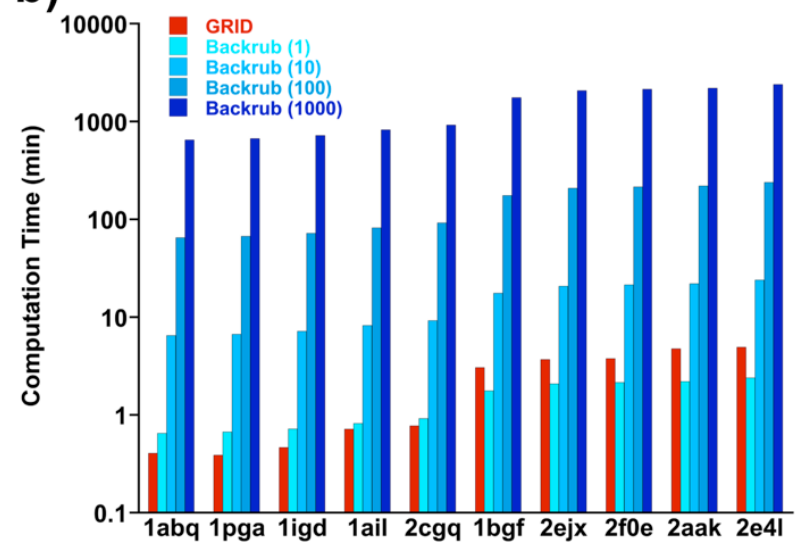

c)

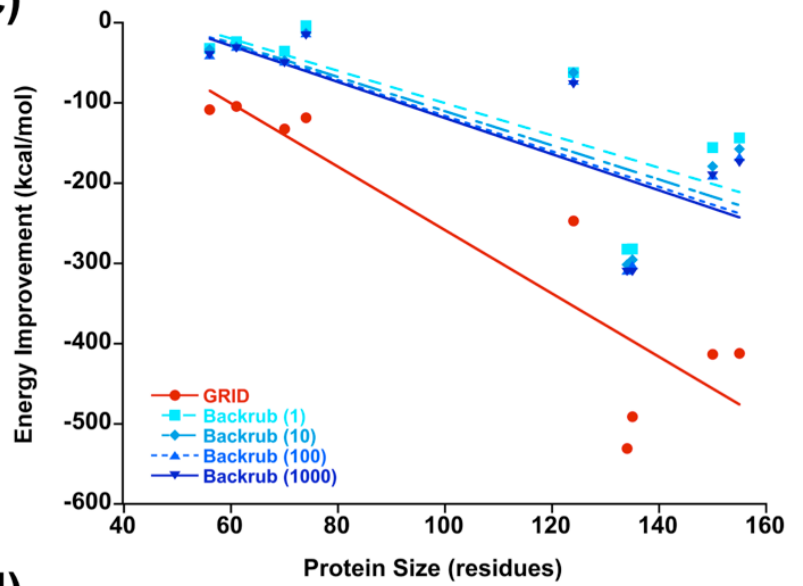

d)

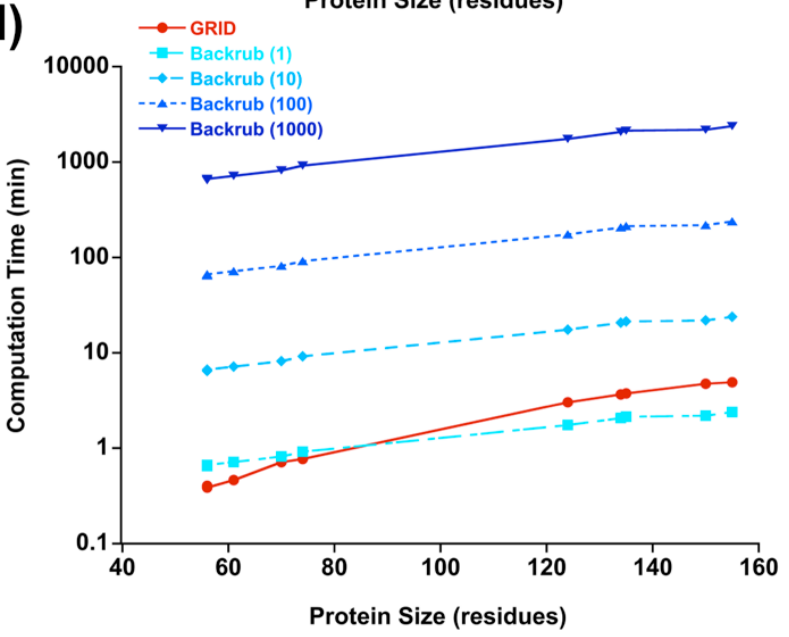

Figure 3. Performance of GRID versus Backrub in refining 10 high-resolution crystal structures from the PDB. Energy improvement (a) and computation time (b) are shown for each of the 10 proteins. For Backrub, which is a stochastic algorithm, values are given for 1, 10, 100, and 1000 trajectories. Energy improvement (c) and computation time (d) are also plotted as a function of protein size. 


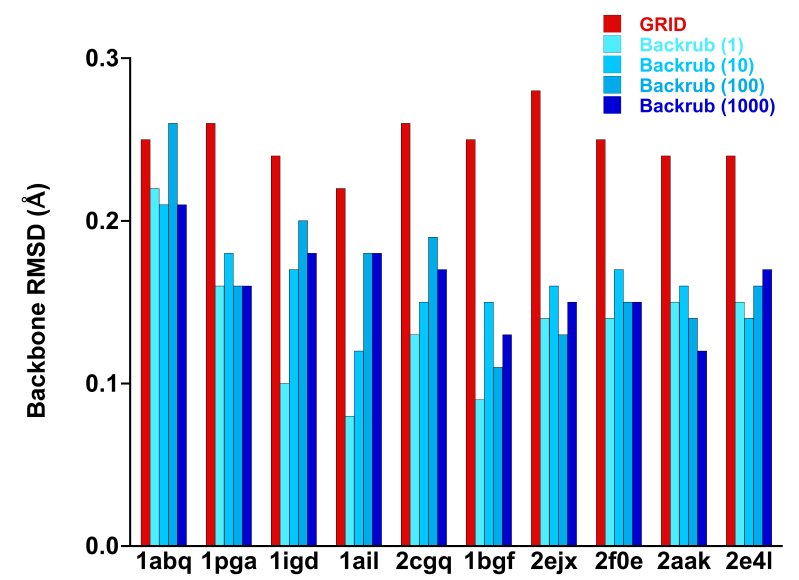

Figure 4. Backbone RMSD with respect to starting crystal structure following refinement with GRID and Backrub. Both algorithms keep the refined structures close to their original topologies. 


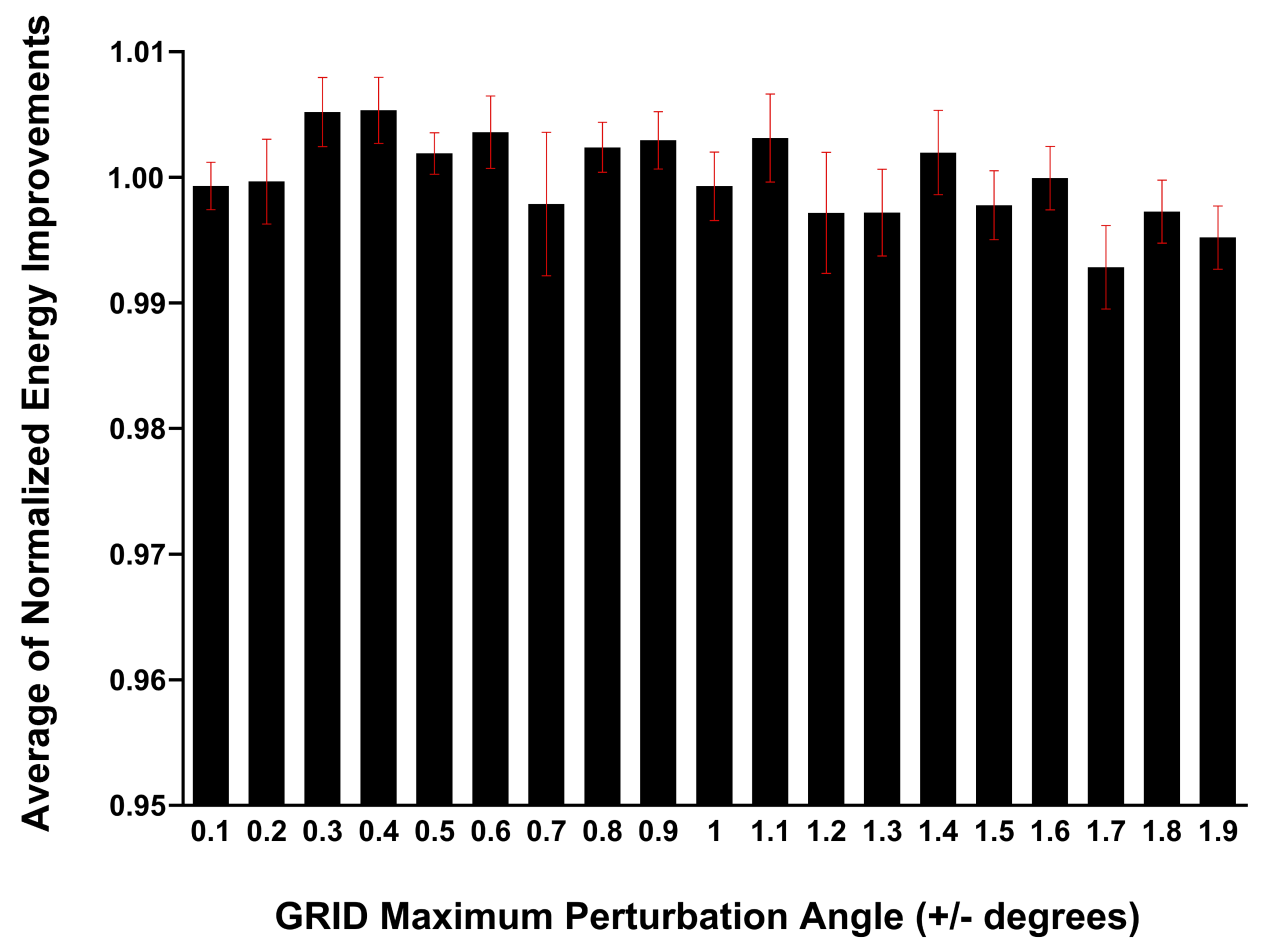

Figure S1. Energy improvement following refinement with GRID using maximum dihedral angle perturbations ranging from $\pm 0.1^{\circ}$ to $\pm 1.9^{\circ}$; a single iteration was performed and the precision level was set to produce a 9-point $\Delta \varphi \times \Delta \psi$ matrix $(3 \times 3)$. For each of the 10 proteins in the test set, the energy improvement $(\mathrm{kcal} / \mathrm{mol})$ was averaged over all the perturbation angles and normalized. Averages of these normalized values for all 10 proteins \pm S.E. are shown. 

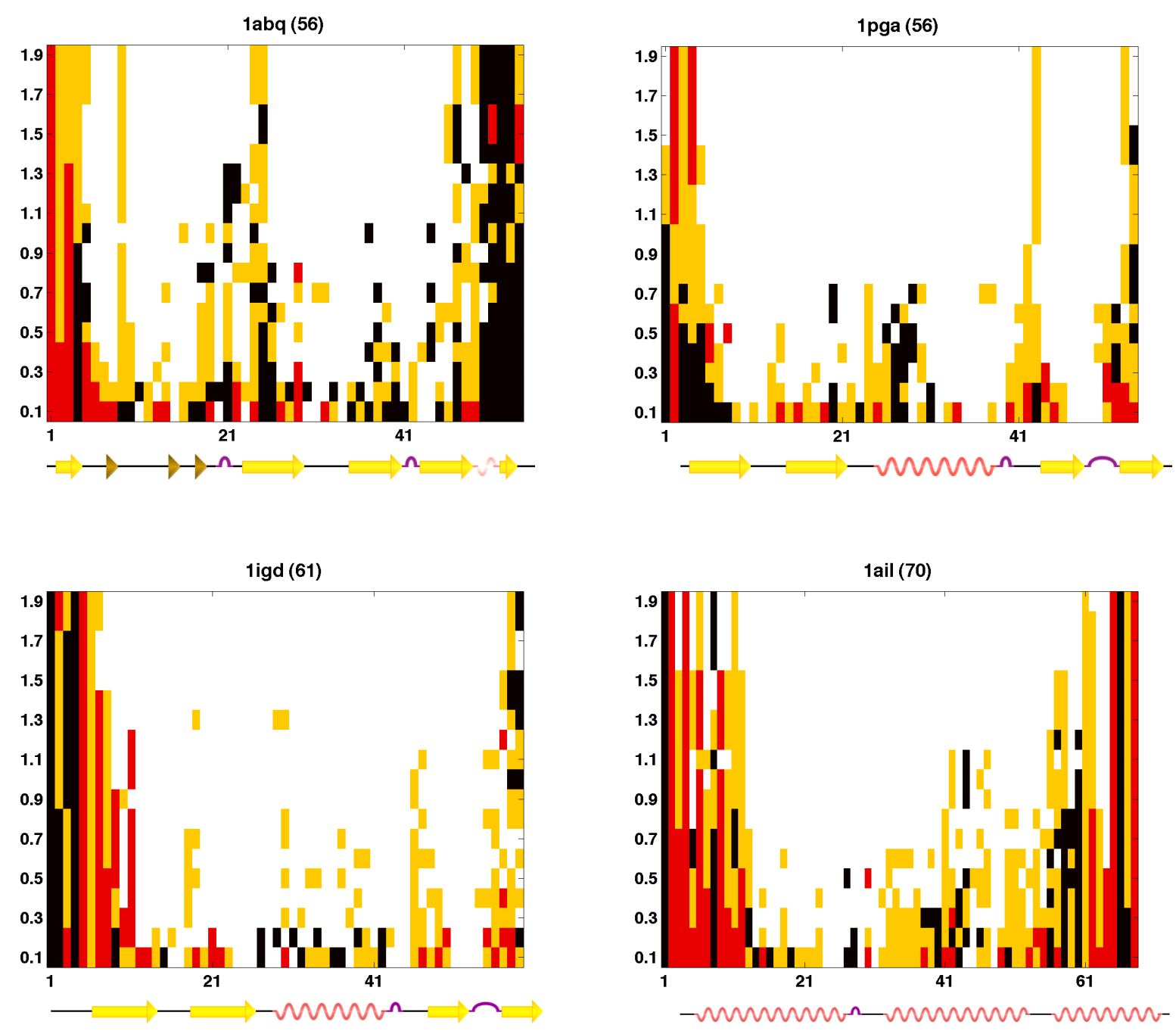

$$
\begin{aligned}
& \square \Delta \phi=\Delta \psi=0 \\
& \square \Delta \phi=0 \text { XOR } \Delta \psi=0 \\
& \square \Delta \phi=\Delta \psi \neq 0 \\
& \square \phi=-\Delta \psi \neq 0
\end{aligned}
$$



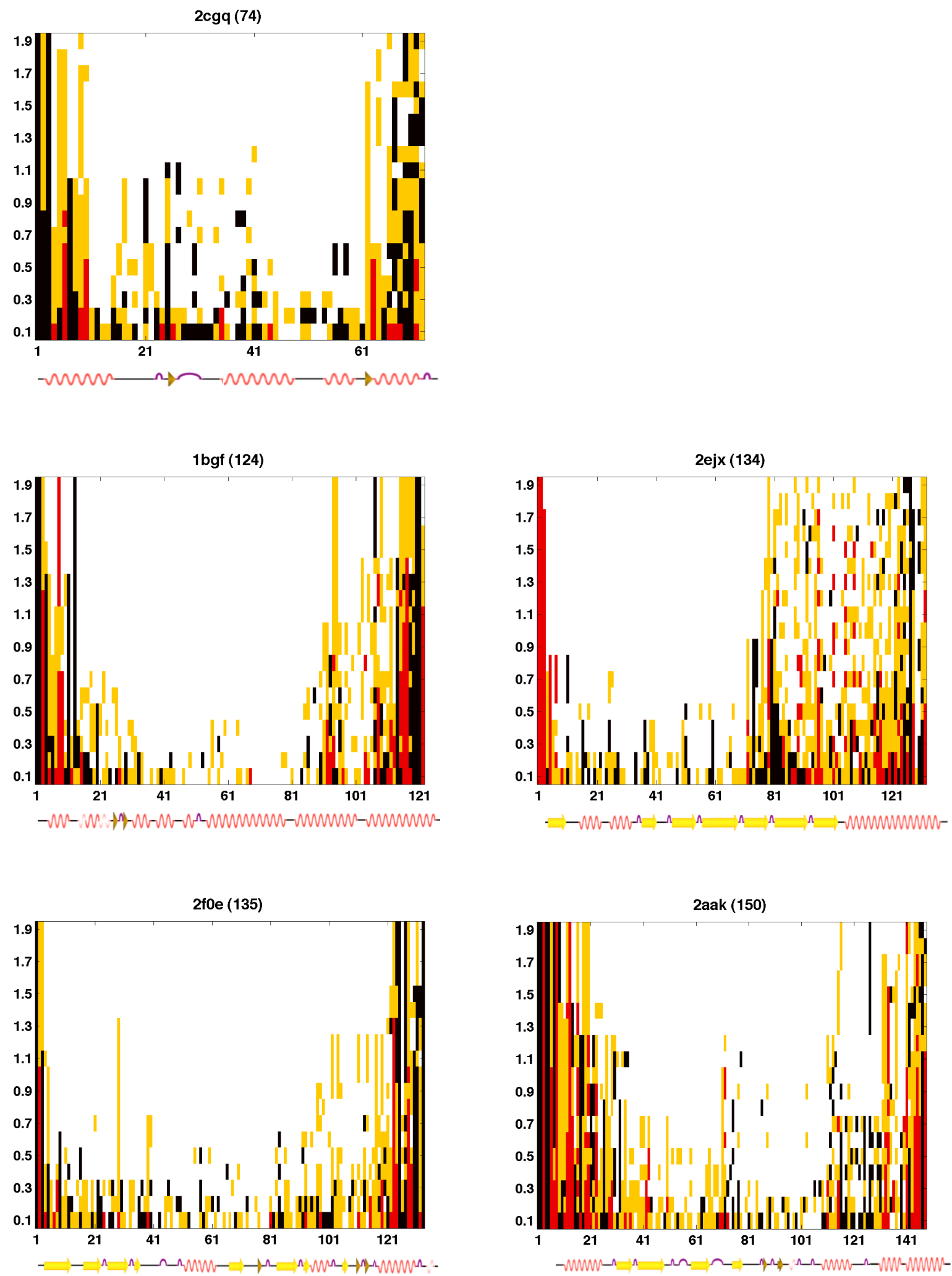


$$
\begin{aligned}
& \square \Delta \phi=\Delta \psi=0 \\
& \square \Delta \phi=0 \text { XOR } \Delta \psi=0 \\
& \square \Delta \phi=\Delta \psi \neq 0 \\
& \square \phi=-\Delta \psi \neq 0
\end{aligned}
$$

\section{2e4l (155)}

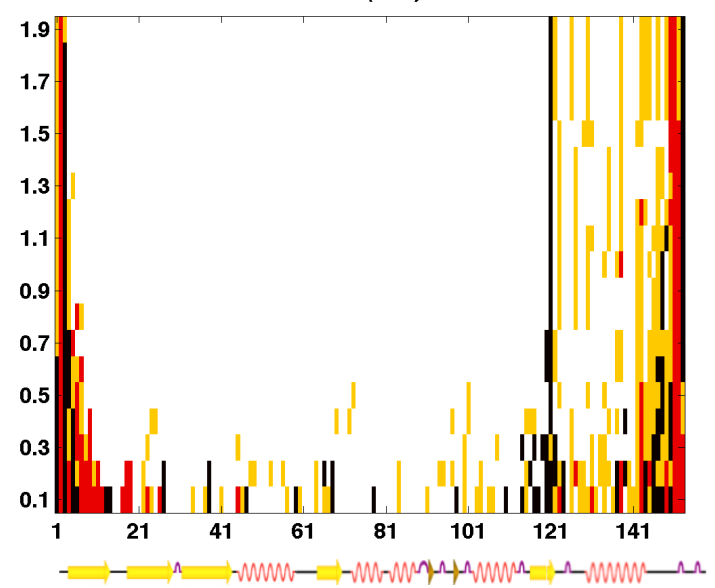

Figure S2. GRID moves plotted as a function of residue number ( $x$ axis) and maximum dihedral angle perturbation (y axis) for each of the 10 proteins tested (PDB ID and length of protein are given at top of each graph). The precision level was always set to form the smallest $\Delta \varphi \times \Delta \psi$ matrix (9 grid points) and a single iteration was performed. The four move types are as follows: type A (white), type B (yellow), type C (red), and type D (black). At larger perturbation angles, moves that cause no backbone movement (type A) become increasingly dominant, and moves that actually perturb the backbone (types B-D) are restricted to residues near the termini. Smaller perturbation angles allow movement to occur more uniformly throughout the entire protein structure. 
Table 1. Proteins used as test set for structure refinement.

\begin{tabular}{lrrccr|} 
PDB ID & $\begin{array}{c}\text { No. of } \\
\text { residues }\end{array}$ & $\begin{array}{c}\text { Resolution } \\
(\AA)\end{array}$ & Class & $\% \alpha$ & $\% \beta$ \\
\hline 1abq & 56 & 2.80 & $\beta$ & 4 & 43 \\
1pga & 56 & 2.07 & $\alpha+\beta$ & 25 & 42 \\
1igd & 61 & 1.10 & $\alpha+\beta$ & 22 & 42 \\
1ail & 70 & 1.90 & $a$ & 80 & 0 \\
2cgq & 74 & 1.83 & $a$ & 38 & 1 \\
1bgf & 124 & 1.45 & $\alpha$ & 79 & 1 \\
2ejx & 134 & 1.79 & $\alpha+\beta$ & 35 & 41 \\
2f0e & 135 & 1.95 & $\alpha+\beta$ & 24 & 28 \\
2aak & 150 & 2.40 & $\alpha+\beta$ & 35 & 19 \\
2e4l & 155 & 2.00 & $\alpha / \beta$ & 34 & 29 \\
\hline aClassifications based & on Dictionary & of Secondary Structure of \\
Proteins (DSSP) & & & & & \\
\hline
\end{tabular}




\section{Chapter 2}

REMCGRID: A Sampling Algorithm for Protein Structure Refinement and Decoy Set Generation 


\section{Abstract}

We present replica exchange Monte Carlo (REMC) ${ }^{\text {GRID }}$, an algorithm that incorporates the core strategies of GRID ${ }^{1}$, a previously developed refinement algorithm, with REMC sampling. GRID uses a deterministic approach to high-resolution refinement, improving the energy of a protein structure by systematically perturbing the backbone dihedrals and side-chain conformations. In this study, REMCGRID was used for two purposes: (1) structure refinement, and (2) generation of decoy structures. In both applications, REMCGRID takes a protein structure as input and generates a conformationally diverse set of low-energy perturbed structures. One can specify the level of dihedral angle perturbations applied and the probability of accepting a perturbed conformation using $\theta^{\max }$ and $k T_{f}$ parameters. For refinement, we used 10 high-resolution $(\leq 2.8 \AA)$ crystal structures from the Protein Data Bank (PDB) ${ }^{2}$. The energy improvements obtained and the computational effort required to achieve them were compared for REMCGRID and GRID alone. When longer compute times are allowed, REMCGRID results in energy improvements that are significantly better than those attained by GRID. For decoy generation, we used 59 highresolution ( $\leq 2.8 \AA$ ) crystal structures from the PDB of proteins ranging in size from $50-150$ residues. We applied REMC ${ }^{\text {GRID }}$ with 25 different parameter sets specifying different $\varphi / \psi$ perturbation angles and acceptance probabilities to generate 472,000 decoys. We computed the backbone RMSD of each decoy with respect to its crystal structure and found that REMCGRID can generate a uniform distribution of perturbed structures covering a broad range of RMSDs $(0-10+\AA)$ that is independent of protein size and class. These characteristics (uniformity and broadness) are important for the proper performance of machine learning algorithms. 
Key words: decoy set generation; protein structure refinement; flexible backbone; energy-based refinement; conformational search; replica exchange Monte Carlo 


\section{Introduction}

Computational modeling of protein structures continues to be essential for advancing research in biology and drug discovery, especially given that experimental structures for many biologically important targets are difficult to obtain. Although investigators have focused efforts on protein structure prediction for over half a century, the accurate and reliable prediction of protein structures still remains a major challenge ${ }^{3}$. A successful structure prediction algorithm must solve two problems: (1) inaccuracies in the force field, and (2) inefficient sampling of the conformational space ${ }^{4}$. The two problems are inter-related; for example, one needs an efficient sampling algorithm in order to validate improvements in a force field ${ }^{5}$. Several groups have worked on developing force fields ${ }^{6-15}$, and creating better sampling methods ${ }^{16-22}$.

One of the major problems with current force fields is that they are too sensitive $e^{6,23}$. Small perturbations in the configuration of a structure can result in large changes in its energy. For instance, a few atomic clashes can result in an unfavorable energy for the entire structure, even though the overall fold might be very close to the native state. This imposes ruggedness on the energy landscape and makes it hard for sampling algorithms to search the conformational space and find the lowest energy states of the structure without getting trapped in local minima. Coarse-grained approaches aim to decrease the sensitivity of current force fields by using a simpler model to describe proteins and their interatomic interactions ${ }^{24}$. To test the validity of any coarse-grained model, one needs a decoy set that covers a broad range of RMSDs so that the model's ability to properly rank the decoys can be tested. We therefore set out to create a decoy-generating algorithm that could provide a similar distribution of perturbed structures spanning a broad range of 
RMSD's for proteins of different sizes and classes. We were able to achieve this by incorporating a previously developed deterministic refinement algorithm (GRID) with replica exchange Monte Carlo sampling in a novel algorithm called REMCGRID.

\section{Results and Discussion}

\section{REMCGRID algorithm}

REMC ${ }^{\text {GID }}$ is a sampling algorithm that takes a three-dimensional protein structure as input and, employing an all-atom force field, perturbs the structure using a move set that allows for backbone and side chain flexibility (see Fig. 1). Three degrees of freedom are considered: $\varphi$ and $\Psi$ dihedral perturbations and side-chain conformation. The algorithm runs multiple Monte Carlo simulations (replicas) in parallel at different temperatures. After a specified number of steps of Monte Carlo simulation, replicas join and stochastically swap configurations based on their energies and the temperatures they are running at according to the Metropolis-Hastings criterion ${ }^{25}$ (see Fig. 6). Each step of Monte Carlo simulation consists of four stages: (1) picking a residue at random, (2) perturbing $\varphi$ and $\psi$ backbone dihedrals of that residue, given a specified maximum perturbation angle range, (3) scanning through all the possible side chain conformations (from a predefined rotamer library) and picking the one that results in the lowest energy for the perturbed structure, and (4) accepting or rejecting the move based on the Metropolis acceptance criterion, given a specified temperature. A rotamer library is used to define a discrete set of conformations for each residue. Although the moves are restricted to local perturbations relative to a single residue, the perturbation selected influences the entire structure. Thus, relatively minor local changes can propagate down the chain resulting in large shifts in the conformation of the protein. 


\section{REMCGRID Improves Structure Refinement}

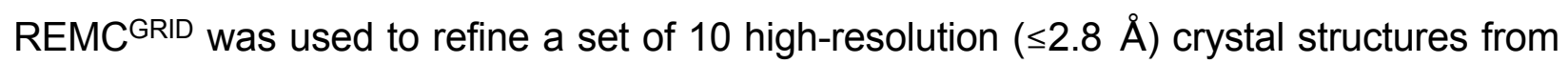
the PDB (Table 1). We calculated the energy improvements attained and the compute times required to achieve them for REMCGRID and for GRID alone. Given enough computational resources, REMCGRID results in structures with significantly better energies than GRID alone (Fig. 2 and Fig. 3). As seen in a plot showing the ratio of computational resources required for REMCGRID to match the level of energy improvement obtained by GRID (see Fig. 4), with longer compute times (number of moves) REMCGRID achieves energy improvements that are up to $\sim 15 \%$ better than GRID alone. Since REMCGRID runs multiple trajectories in parallel and the compute time of each trajectory is comparable to that of GRID (GRID takes 1-2 minutes, since it is deterministic and therefore just runs once), on a large cluster, REMC ${ }^{\text {GRID }}$ can get this $15 \%$ improvement with an overall compute time that is $\sim 3$-fold that of GRID; i.e., in $\sim 2-6$ minutes. Low score decoys generated for one of the 10 protein structures (pdbID 1pga) is shown in Fig. 7 and Fig. 8. 


\section{REMCGRID Generates Uniform Distributions of Diversely Perturbed Decoys}

We used REMCGRID to generate a set of decoys based on 59 high-resolution $(\leq 2.8 \AA \AA)$ crystal structures from the PDB. These 59 structures included proteins ranging in size from $50-150$ residues and is the same dataset used by Das et al. for decoy generation with Rosetta ${ }^{26}$. We applied REMCGRID with 25 different parameter sets specifying 5 different $\varphi / \psi$ perturbation angles and 5 different acceptance probabilities. For each pair of parameters, we generated 320 decoys for each of the 59 proteins, for a total of 472,000 decoys. We found that smaller perturbations of $\varphi$ and $\psi$ result in smaller deviations in structure. Moreover, higher simulation temperatures caused the acceptance of more moves that worsen the energy of the structure, resulting in more diverse and more perturbed decoy sets. The parameter set with the largest $\varphi / \psi$ perturbations and the least restricting $\mathrm{k} \mathrm{T}_{\mathrm{f}}$ values resulted in the most perturbed decoy structures. These decoys covered the broadest range of RMSD's (including $10+\AA$ ) and showed uniform broadness across protein size. These characteristics (uniformity and broadness) are important for the proper performance of machine learning algorithms. We planned to use this decoy set to train a machine learning classifier (SVM), which would be used as a scoring function for structure prediction (see Chapter 3).

\section{REMC ${ }^{\text {GRID }}$ generates more uniform decoys than Rosetta}

In generating decoys, Rosetta starts from an unfolded polypeptide chain and after several rounds of full structure prediction, produces a set of low energy conformations ${ }^{27}$. In contrast, REMCGRID starts from the crystal structure and systematically and gradually increases the energy of the structure to generate near-native, partially-folded, and unfolded 
decoy structures. REMCGRID uses an all-atom force field to ensure that structures with severe clashes are not generated.

We compared our most perturbed REMCGRID decoys (those produced with the largest $\varphi / \Psi$ perturbations and the least restricting $k T_{f}$ values) with those obtained using Rosetta for broadness (range of backbone RMSDs) and uniformity (distribution of RMSDs across different protein structures). The REMCGRID decoys are very uniform: the median value is $\sim 1 \AA$ and is very close to 3 percentile value, and the most perturbed structures (98 percentile) rarely surpass $12 \AA$ RMSD (Fig. 10). In contrast, the Rosetta decoys are quite varied: the median typically ranges from $5-10 \AA$, and the 98 percentile values are much higher, with some decoys reaching $>30 \AA$ RMSD. REMCGRID decoys show adequate broadness $(0$ to $10+\AA)$ and have distributions that are fairly consistent, whereas Rosetta decoys show very large differences in their distributions. Thus, REMC GRID can generate a uniform distribution of perturbed structures covering a broad range of RMSDs that is independent of protein size and class.

\section{Methods}

\section{REMCGRID implementation and parameterization}

The REMCGRID algorithm was developed in $\mathrm{C}++$ and incorporated into the phoenix protein design software package. Standard Message Passing Interface (MPI) was used for multiprocessor parallelization. The phoenix force field with default parameters was used to calculate the energy of the protein conformations. Side-chain conformations were selected from the Dunbrack backbone-dependent rotamer library ${ }^{28}$.

REMCGRID takes as input six main parameters: (1) the maximum perturbation angle $(\Delta \varphi, \Delta \psi)$, (2) number of parallel replicas, (3) number of Monte Carlo steps before 
swapping configurations, (4) number of rounds of running Monte Carlo simulations, (5) lowest temperature replica, and (6) highest temperature replica. Values for maximum $\Delta \varphi$ and $\Delta \psi$ ranged from $\pm 2^{\circ}$ to $\pm 10^{\circ}$; the number of parallel replicas was 80 ; the number of Monte Carlo steps before swapping configuration was 100; number of rounds of Monte Carlo was 20; lowest temperature was $\mathrm{kT}_{\mathrm{i}}=0.5$; and highest temperature ranged from $k T_{f}=1$ to $k T_{f}=9$. The final set of parameters was obtained after trying REMCGRID with several different values for each parameter and computing the RMSDs of the resulting decoys.

\section{Acknowledgments}

This work was supported by the Defense Advanced Research Projects Agency (DARPA) and a Department of Defense National Security Science and Engineering Faculty Fellowship (S.L.M).

\section{References}

1. Chitsaz, M.; Mayo, S. L. J Comput Chem 2013, 34(6), 445-450.

2. Bernstein, F. C.; Koetzle, T. F.; Williams, G. J.; Meyer, E. F., Jr.; Brice, M. D.; Rodgers, J. R.; Kennard, O.; Shimanouchi, T.; Tasumi, M. Eur J Biochem 1977, 80(2), 319-324.

3. Dill, K. A.; MacCallum, J. L. Science 2012, 338(6110), 1042-1046.

4. Summa, C. M.; Levitt, M. Proc Natl Acad Sci U S A 2007, 104(9), 3177-3182.

5. Best, R. B.; Zhu, X.; Shim, J.; Lopes, P. E.; Mittal, J.; Feig, M.; Mackerell, A. D., Jr. J Chem Theory Comput 2012, 8(9), 3257-3273.

6. Ponder, J. W.; Case, D. A. Adv Protein Chem 2003, 66, 27-85. 
7. Vanommeslaeghe, K.; Hatcher, E.; Acharya, C.; Kundu, S.; Zhong, S.; Shim, J.; Darian, E.; Guvench, O.; Lopes, P.; Vorobyov, I.; Mackerell, A. D., Jr. J Comput Chem 2010, 31(4), 671-690.

8. Hsieh, M. J.; Luo, R. J Phys Chem B 2010, 114(8), 2886-2893.

9. Marrink, S. J.; Risselada, H. J.; Yefimov, S.; Tieleman, D. P.; de Vries, A. H. J Phys Chem B 2007, 111(27), 7812-7824.

10. Periole, X.; Marrink, S. J. Methods Mol Biol 2013, 924, 533-565.

11. Yesylevskyy, S. O.; Schafer, L. V.; Sengupta, D.; Marrink, S. J. PLoS Comput Biol 2010, 6(6), e1000810.

12. Li, X.; Wu, X.; Wu, G. J Theor Biol 2014.

13. Berhanu, W. M.; Jiang, P.; Hansmann, U. H. Phys Rev E Stat Nonlin Soft Matter Phys 2013, 87(1), 014701.

14. Vendruscolo, M. J Biol Phys 2001, 27(2-3), 205-215.

15. Koga, N.; Takada, S. J Mol Biol 2001, 313(1), 171-180.

16. Daidone, I.; Amadei, A.; Roccatano, D.; Nola, A. D. Biophys J 2003, 85(5), 2865-2871.

17. Chen, J.; Im, W.; Brooks, C. L., 3rd. J Comput Chem 2005, 26(15), 1565-1578.

18. Frembgen-Kesner, T.; Elcock, A. H. J Mol Biol 2006, 359(1), 202-214.

19. Stumpff-Kane, A. W.; Maksimiak, K.; Lee, M. S.; Feig, M. Proteins 2008, 70(4), 13451356.

20. Jiang, P.; Yasar, F.; Hansmann, U. H. J Chem Theory Comput 2013, 9(8).

21. Yang, Y.; Liu, H. J Comput Chem 2006, 27(13), 1593-1602.

22. Zheng, Q.; Rosenfeld, R.; DeLisi, C.; Kyle, D. J. Protein Sci 1994, 3(3), 493-506. 
23. Price, D. J.; Brooks, C. L., 3rd. J Comput Chem 2002, 23(11), 1045-1057.

24. Tozzini, V. Curr Opin Struct Biol 2005, 15(2), 144-150.

25. Haario, H.; Saksman, E.; Tamminen, J. Bernoulli 2001, 7(2), 223-242.

26. Das, R.; Qian, B.; Raman, S.; Vernon, R.; Thompson, J.; Bradley, P.; Khare, S.; Tyka, M. D.; Bhat, D.; Chivian, D.; Kim, D. E.; Sheffler, W. H.; Malmstrom, L.; Wollacott, A. M.; Wang, C.; Andre, I.; Baker, D. Proteins 2007, 69 Suppl 8, 118-128.

27. Das, R.; Baker, D. Annu Rev Biochem 2008, 77, 363-382.

28. Dunbrack, R. L., Jr.; Karplus, M. J Mol Biol 1993, 230(2), 543-574. 
input: $k T_{i}, k T_{f}$, replicas, MCsteps, $\theta^{\max }$, rounds, struct

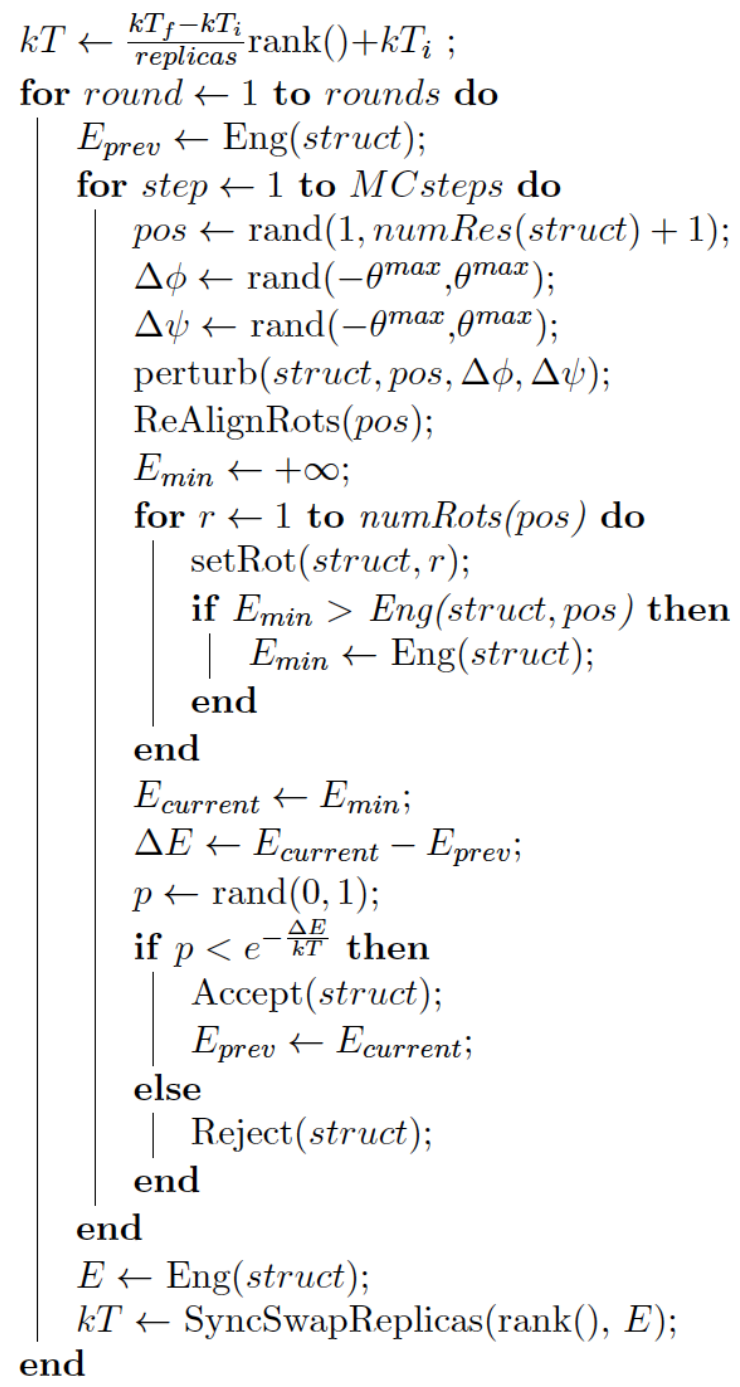

Fig 1. REMCGRID algorithm pseduocode; the algorithm takes a crystal structure and a parameter set as input and generates a set of low energy decoys as output. Level of perturbation depends on the parameter set. 


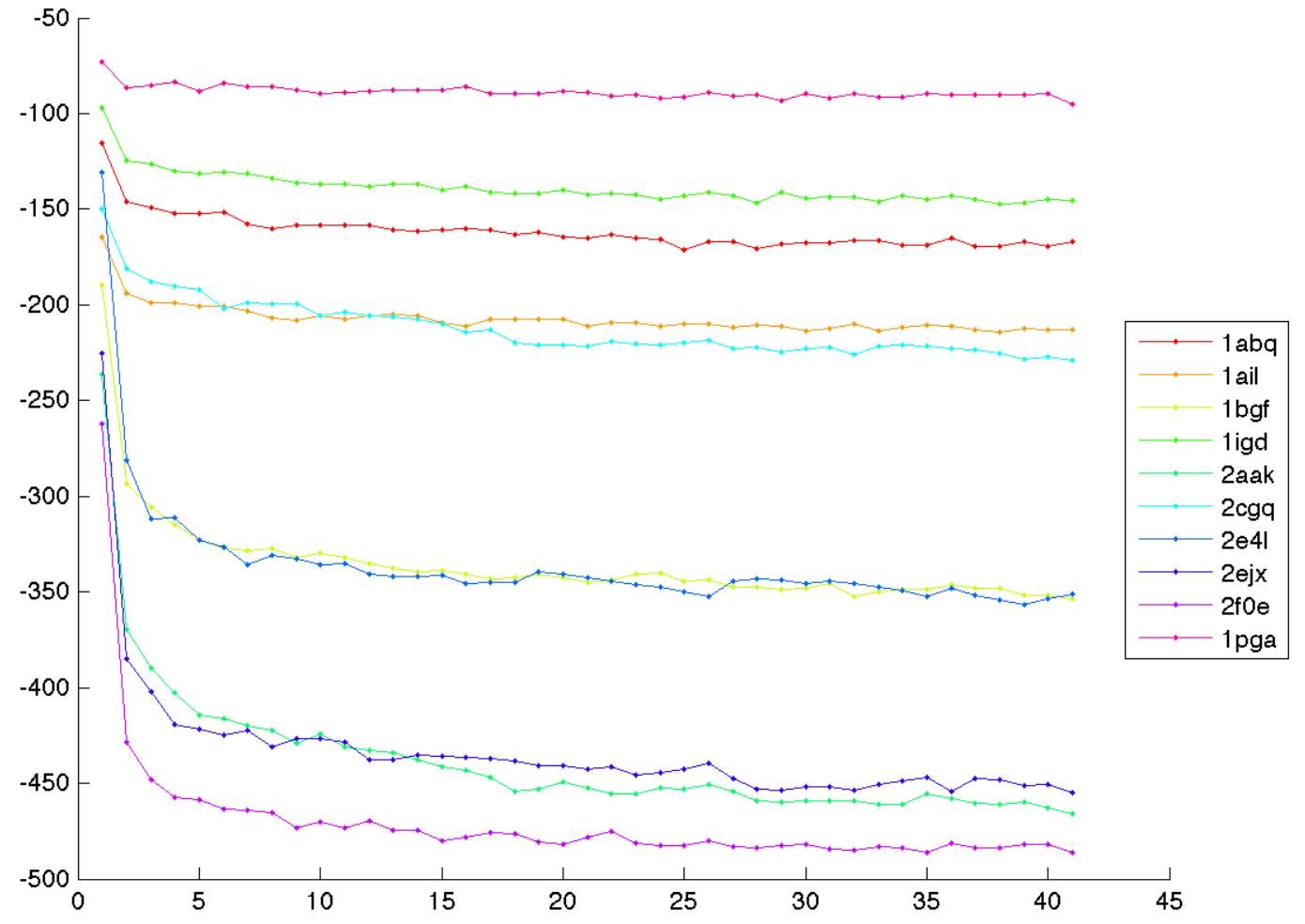

Fig 2. The energy improvement of 10 single-chain high-resolution crystal structures using REMCGRID employing phoenix energy force field. 


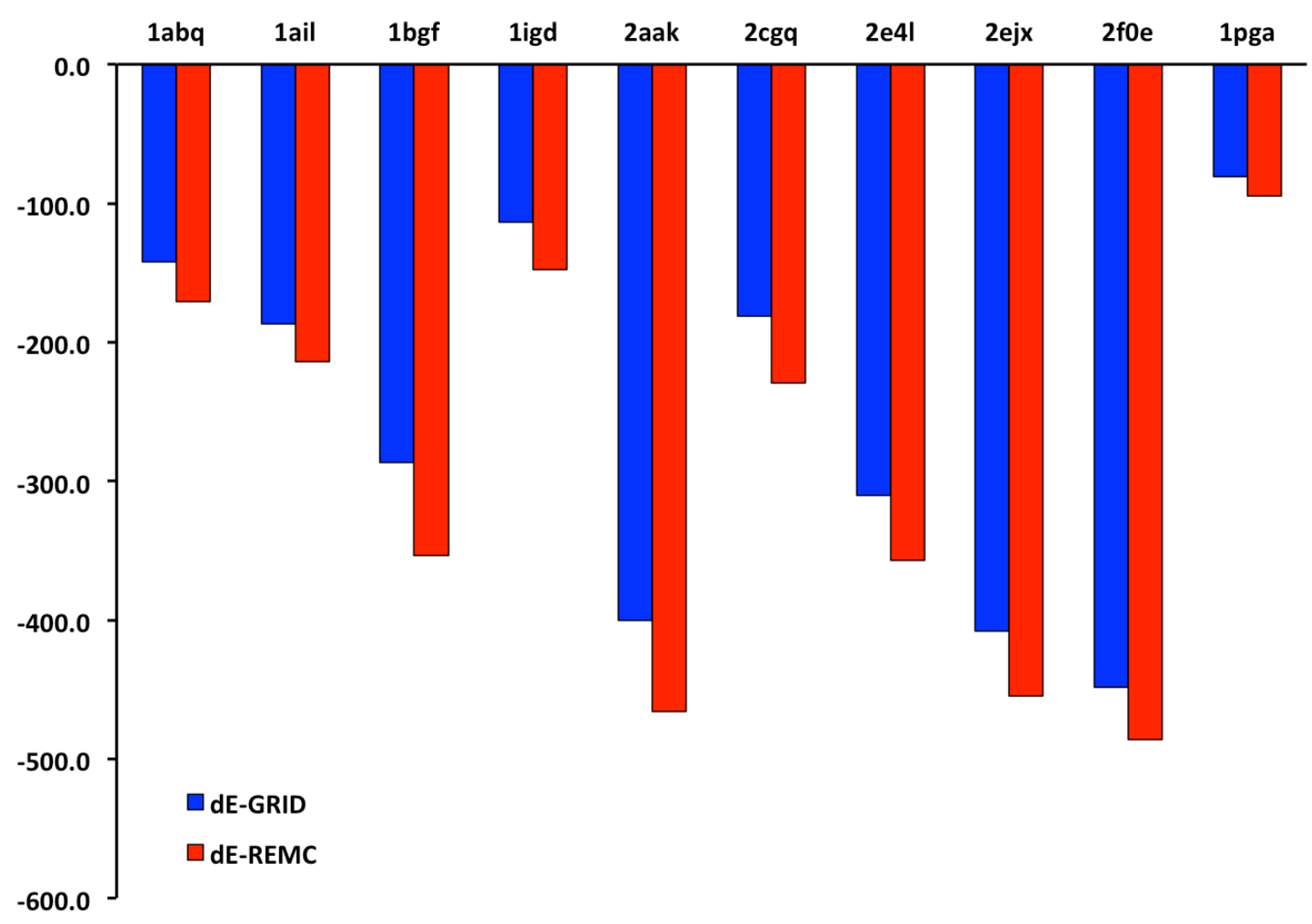

Fig 3. REMCGRID energy improvement compared to GRID energy improvement; REMCGRID results in significantly more energy improvements than GRID. 


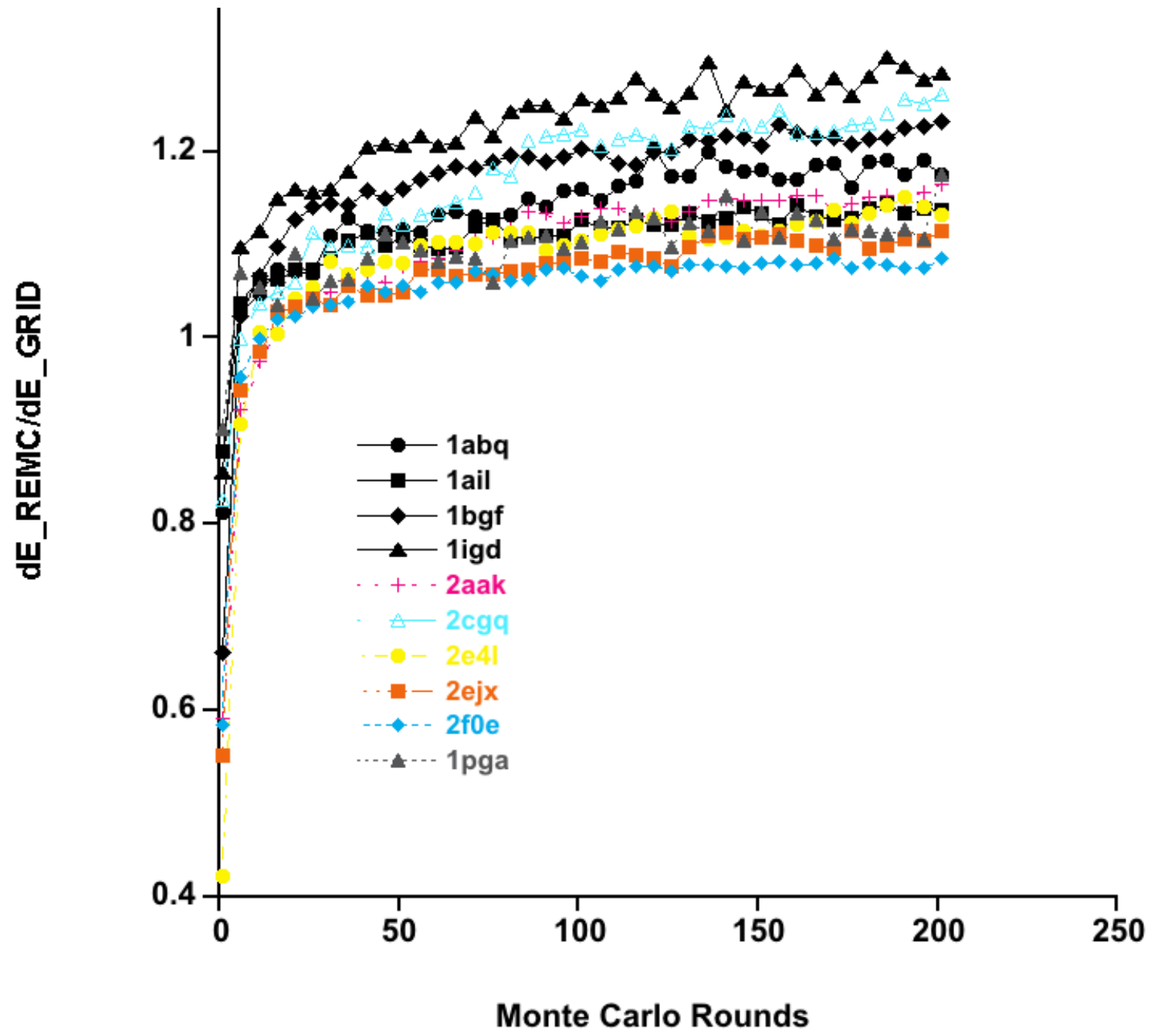

Fig 4. The ratio of energy improvement that REMCGRID achieves compared to the energy improvement attained by GRID based on the number of steps of Monte Carlo simulation. 


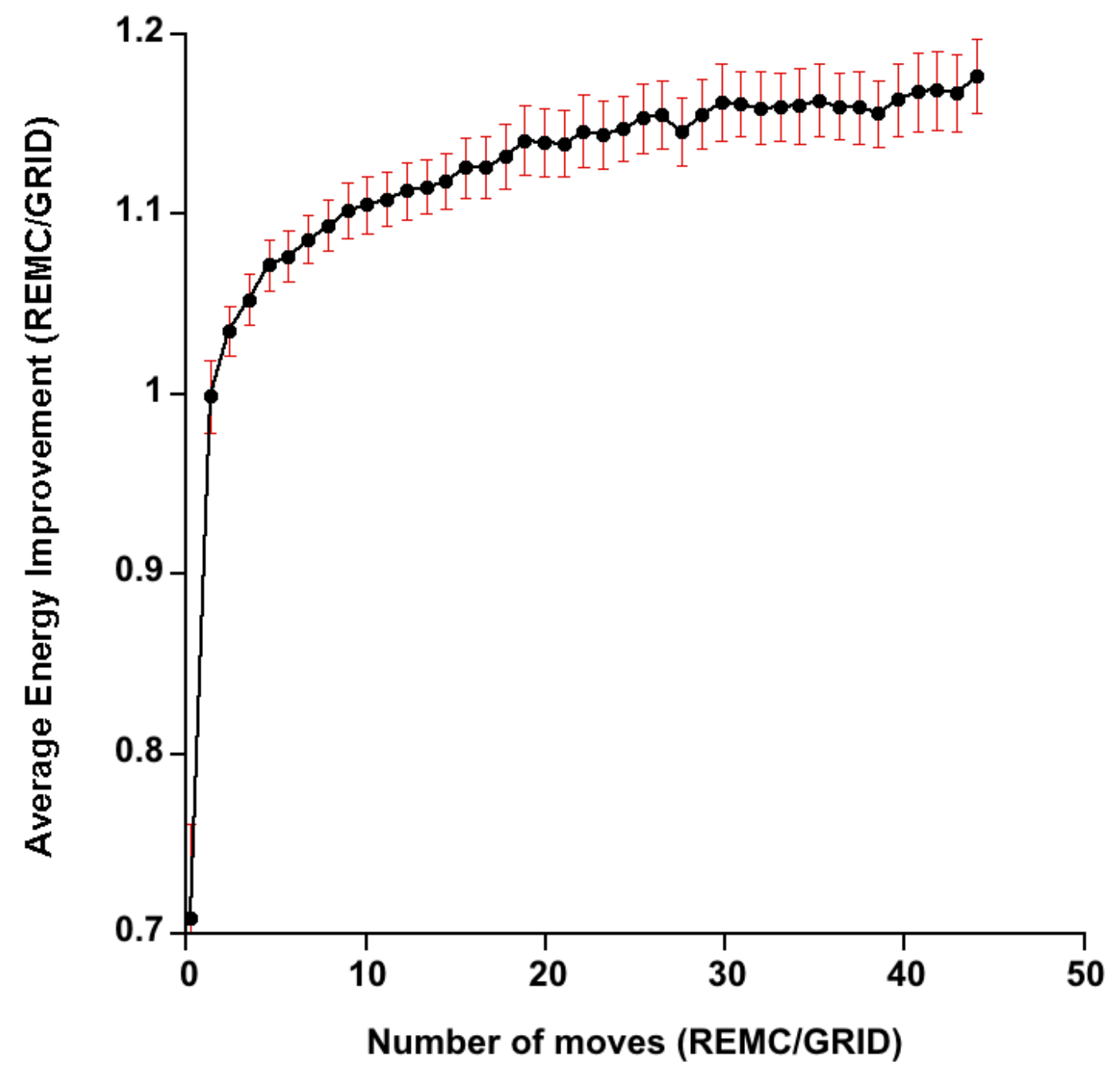

Fig 5. Computational effort comparison between REMCGRID and GRID; REMCGRID by spending more computational effort can achieve higher energy improvements. REMC ${ }^{\text {GRID }}$ continues to improve the energy improvement by being allowed to spend more computational resources. 


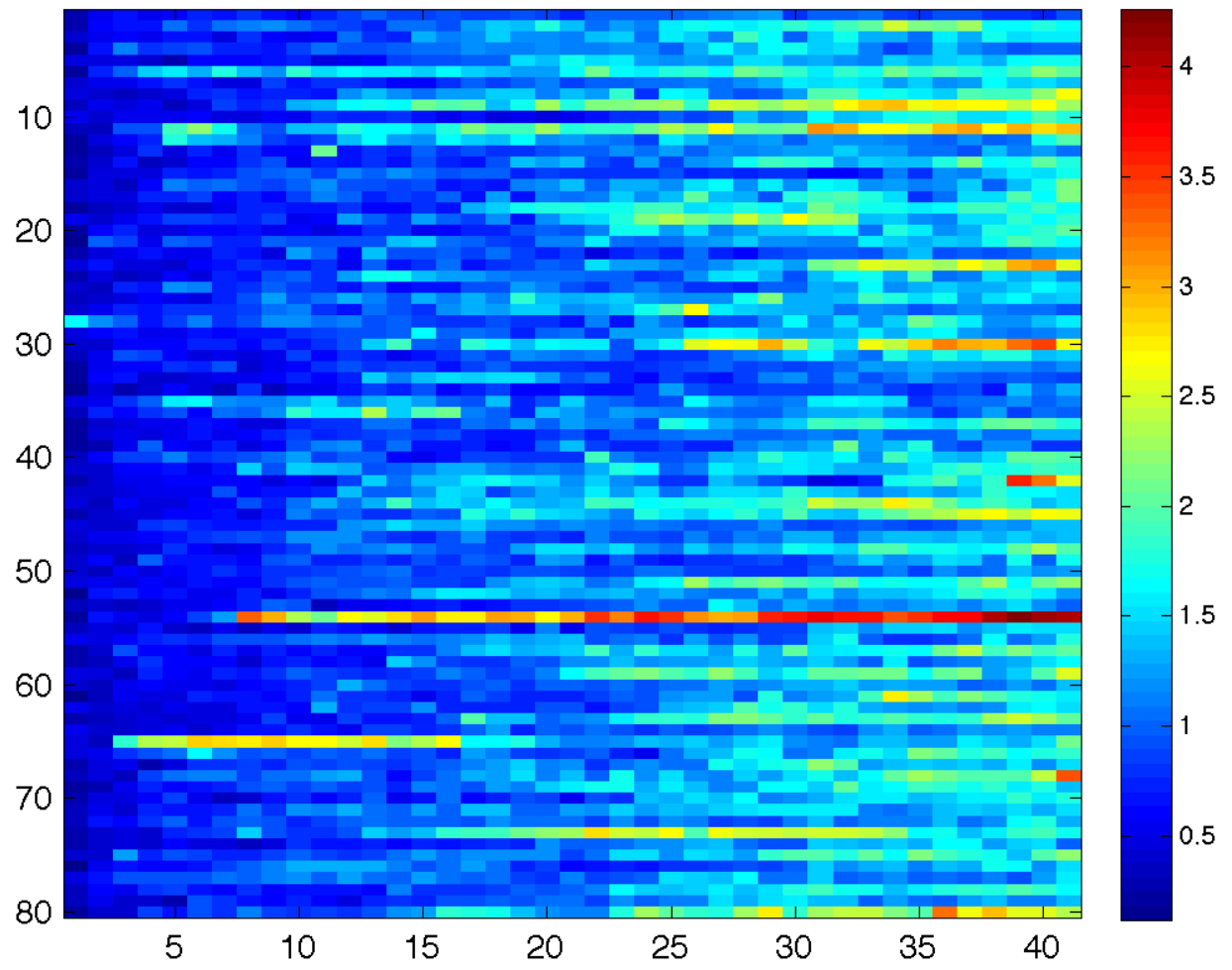

Fig 6. Backbone rmsds of 80 parallel replicas; 40 rounds of swaps between replicas is shown; protein $G$ with pdbID "1pga" is the protein we used in this simulation. 


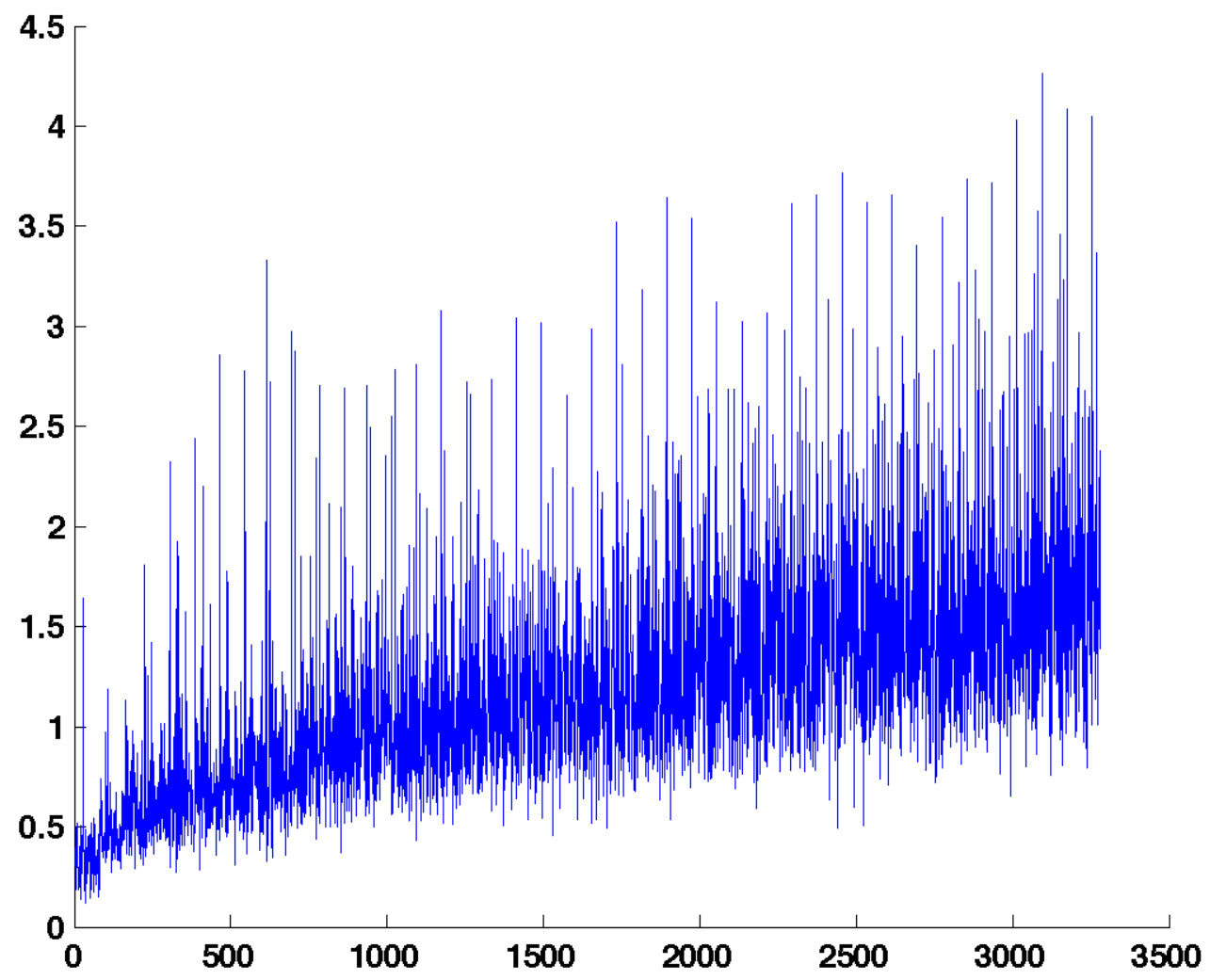

Fig 7. Backbone rmsds of decoys (protein G, 1pga); backbone rmsd increases as the number of iterations increases in this simulation. Parameters are set to generate a diverse set of decoys. 


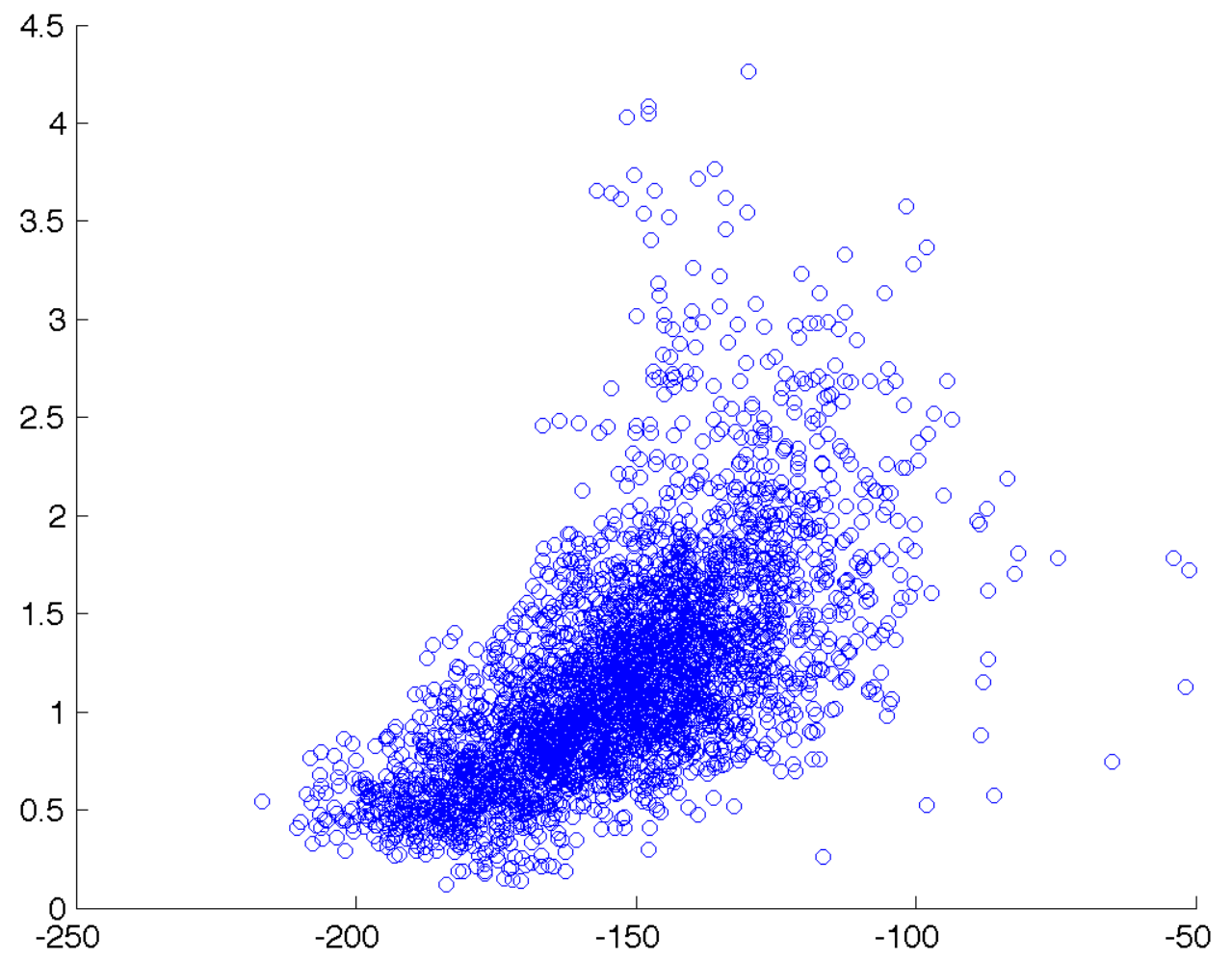

Fig 8. Backbone rmsd vs. energy of decoys generated; gradual increase in the energy of the structure results in a funnel like distribution of backbone RMSDs vs. energies (pdbID 1pga). 

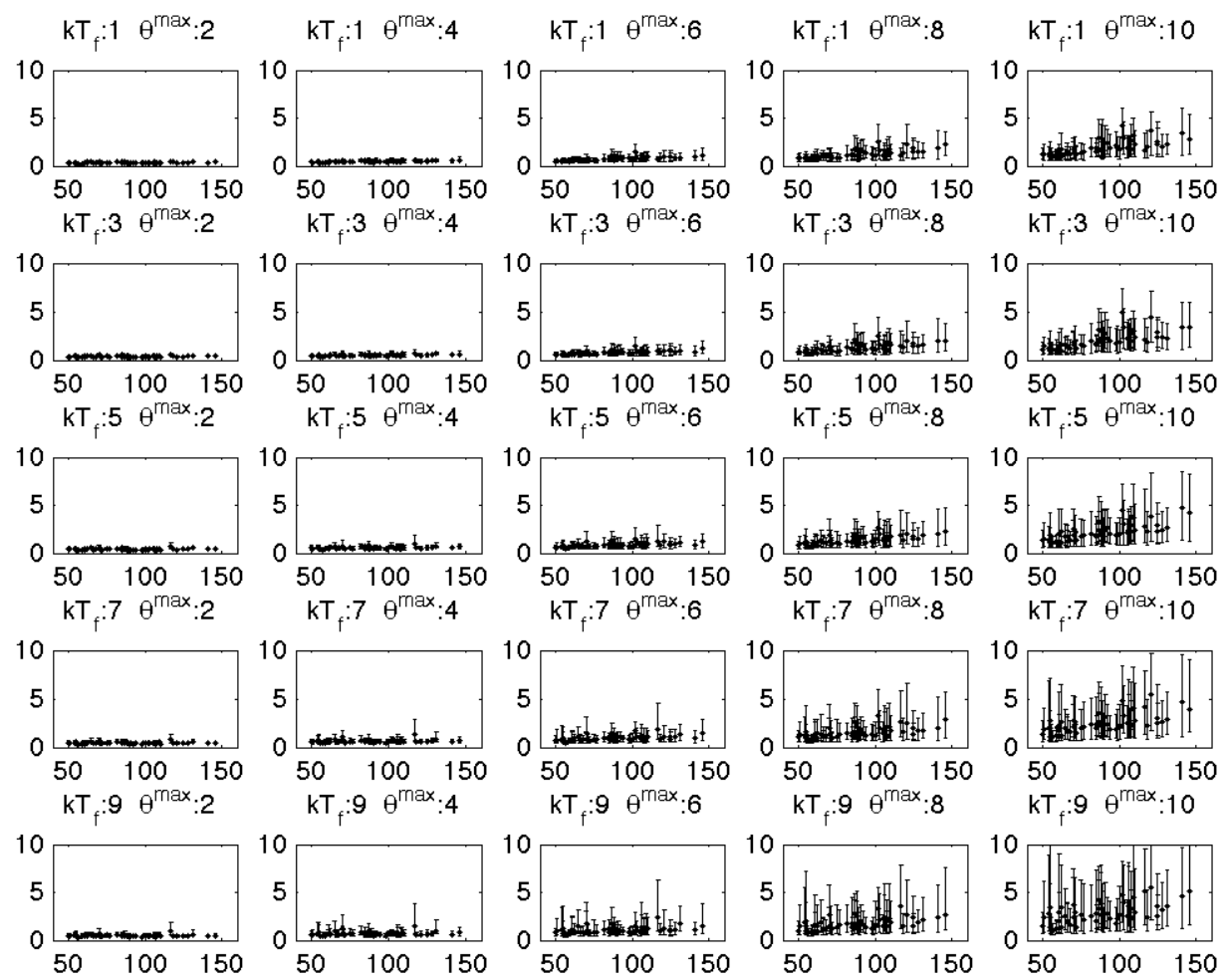

Fig 9. Backbone rmsd distribution of decoy sets generated by REMCGRID. In this figure we have generated 25 different decoy sets by varying two parameters of the algorithm $\left(k T_{f}\right.$ and $\left.\theta\right)$. Each decoy set is depicted in a box; the $x$-axis is the size of protein in terms of number of residues, and the $y$-axis is the backbone rmsd. Each line corresponds to one single protein structure and the decoys generated for that specific structure. The line is marked by three points of 25,50 , and 75 percentiles. 

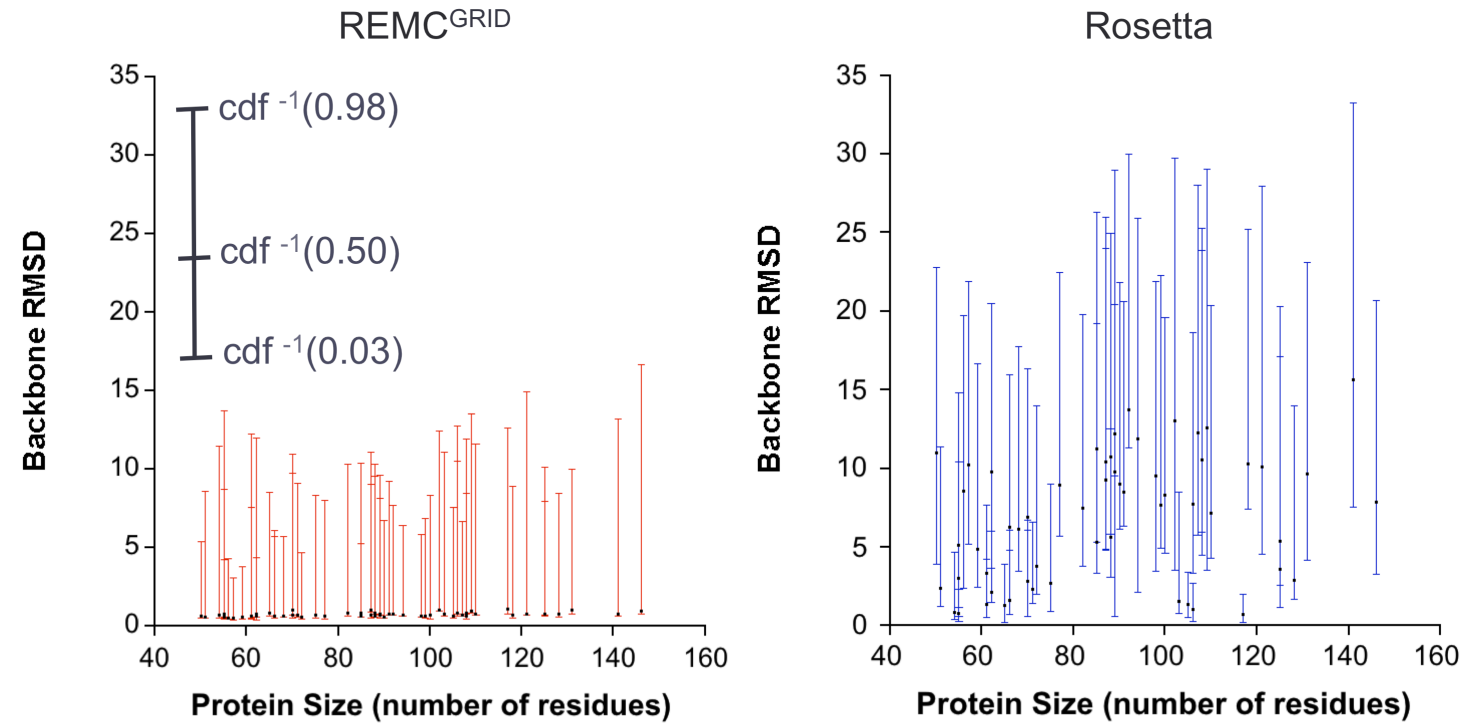

Fig 10. Comparison of Rosetta decoys and REMCGRID decoys; REMCGRID decoys are much more uniformly distributed due to gradual increase of the energy of the structures; Rosetta on the other hand produces set of decoys by performing a full structure prediction and picking the lowest energy decoys. Each line is marked at three points, the lowest point corresponds to 3 percentile, the middle mark corresponds to 50 percentile and the top most mark corresponds to 98 percentile. 


\begin{tabular}{|c|c|c|c|c|c|}
\hline PDB ID & No. of residues & Resolution $(\AA)$ & Class & $\% \alpha$ & $\% \beta$ \\
\hline $1 \mathrm{abq}$ & 56 & 2.80 & $\beta$ & 4 & 43 \\
\hline 1pga & 56 & 2.07 & $a+\beta$ & 25 & 42 \\
\hline 1igd & 61 & 1.10 & $a+\beta$ & 22 & 42 \\
\hline 1ail & 70 & 1.90 & $a$ & 80 & 0 \\
\hline $2 \mathrm{cgq}$ & 74 & 1.83 & $a$ & 38 & 1 \\
\hline $1 \mathrm{bgf}$ & 124 & 1.45 & $a$ & 79 & 1 \\
\hline 2ejx & 134 & 1.79 & $a+\beta$ & 35 & 41 \\
\hline 2f0e & 135 & 1.95 & $a+\beta$ & 24 & 28 \\
\hline 2aak & 150 & 2.40 & $a+\beta$ & 35 & 19 \\
\hline 2e4l & 155 & 2.00 & $\alpha / \beta$ & 34 & 29 \\
\hline
\end{tabular}

Table 1. Ten high-resolution crystal structures that were used in testing REMCGRID refinement. 


\section{Chapter 3}

A Machine Learning Approach to Improving Structure Prediction by Incorporating Coarse-Grained Features 


\section{Abstract}

Although considerable progress has been made in the last few decades, the ability to predict the three-dimensional structure of a protein from its amino acid sequence remains a major challenge in structural biology. A successful energy-based protein structure prediction algorithm must address two problems: (1) inaccuracies in the force field, and (2) inefficient sampling of conformational space. The all-atom force fields currently available are too sensitive-even very minor perturbations in the coordinates of a protein can result in enormous energy fluctuations. This results in a very rugged energy landscape that is difficult to explore. One approach to solving this problem is to incorporate coarse-grained features into the force field. Here, we develop a coarse-grained model and use it to calculate a coarse-grained (CG)-score for a set of decoy structures. Three additional features were also calculated: surface area, volume, and all-atom energy. Decoys were classified into four groups based on their backbone RMSD relative to the crystal structure. A support vector machine (SVM) classifier was then trained to predict the class of each decoy in three different training experiments. The first two used either all-atom energy or CG-score as the sole feature for training and the third combined all four features (all-atom energy, CG-score, surface area, and volume). The CG-score alone produced better results than the all-atom energy alone, but the combination of all four features significantly improved the ability of the SVM to correctly classify the decoys. 


\section{Introduction}

Efforts aimed at predicting the structure of a protein from its amino acid sequence have been ongoing for over 50 years, and although much progress has been made, the accurate and reliable prediction of protein structures still presents many challenges ${ }^{1}$. For an energy-based structure prediction algorithm to be successful, it must deal with two problems: (1) inaccuracies in the force field, and (2) inefficient sampling of the conformational space $^{2}$. Force field development has been ongoing for many years, and several research groups are attempting to develop simple yet relevant models that describe the physics of protein folding 345678 . The simplicity of the models is crucial as compute power is limited and a simpler model can reduce the calculation time required to adequately sample the possible conformations ${ }^{7}$.

One of the problems with current force fields is that they are too sensitive; that is, small perturbations in the configuration of a structure can result in large differences in its energy 95 . This results in a very rugged energy landscape, which makes it difficult for sampling algorithms to search conformational space to find the lowest energy structure without being trapped in local minima. A smoother energy landscape is therefore desirable and would in fact mimic that of natural proteins, whose folding rate suggests a smooth landscape ${ }^{10}$. Coarse-grained models aim to alleviate the sensitivity of current force fields ${ }^{11} 7$ 12. Here, we describe a simple coarse-grained model that was devised for use in training a machine learning classifier. This coarse-grained model was used to calculate a CGscore for a set of decoy structures. Similarly, we computed three other features for the decoys: surface area, volume, and all-atom energy. We classified the decoys into four 
groups based on backbone rmsd from the crystal structure. We then trained a support vector machine (SVM) classifier on these features to predict the class of each decoy. Three SVM training experiments were conducted: (1) with all-atom energy as the sole feature, (2) with CG-score as the sole feature, and (3) with all four features used simultaneously (CG-score, all-atom energy, surface area, and volume). We found that the combination of all four features was more predictive than either all-atom energy or CG-score alone.

\section{Results and Discussion}

\section{A simple coarse-grained model}

To overcome the sensitivity of an all-atom force field, we devised a coarse-grained model for use in training a machine-learning scheme. Our coarse-grained model uses a very simple representation of protein structure, simplifies the calculation of inter-residue interactions by grouping amino acid types, and calculates derivative energies explicitly, all of which speed up the sampling of conformational space. Unlike other coarse-grained models, it also favors stabilizing long-range interactions, which are typically not taken into account.

The backbone of the protein structure is modeled by connecting the C-alpha (CA) atoms with straight lines, and side chains are modeled as spheres connected to the corresponding CA atoms on the backbone (see Fig. 1). The center of mass of the side chain is considered to be the center of the sphere. For each residue type, we calculated the average sphere radius by processing more than 3,500 high-resolution crystal structures extracted from the Protein Data Bank ${ }^{13}$. Similarly, we calculated the average length of the 
bond (straight line) connecting two consecutive CA atoms on the backbone. This model can be used to score any three-dimensional configuration of a protein structure. The CGscore for a given protein structure is computed by summing all the pairwise energies of all the residues. The pairwise energy of two residues is the sum of three energies: (a) the backbone-backbone energy $\left(E_{b b}\right),(b)$ the sidechain-backbone energy $\left(E_{s b}\right)$, and $(c)$ the sidechain-sidechain energy $\left(E_{s s}\right)$. The $E_{b b}$ for two residues is based on the distance between their CA atoms, with a penalty applied if the distance is less than $4.2 \AA$ (Fig. 2A). The $E_{s b}$ is similarly based on the distance between the center of the sphere of the first residue and the CA atom of the other residue. A clash between the sphere and the backbone results in a penalty (Fig. 2B). $E_{s s}$ is based on the distance between the centers of mass of the two residues, their radii, and which of 7 amino acid groups they belong to (categorized by size and biophysical properties) (see Table 1 and Fig. 2C). Basically, if the two residues are clashing, a penalty is applied; if they are far away from each other, there is no penalty; and if they are close but not clashing, a score is computed that depends on their amino acid groups (see Table 2 and Fig. 3). Note that $E_{s s}$ is scaled up by sqrt(i-j) factor, to favor long-range stabilizing contacts. Long-range interactions that are not stabilizing are not favored (this factor is simply +1 ).

\section{Generation of Decoy Structures}

As described in Chapter 2, to generate decoys, we chose 59 high-resolution single chain crystal structures from the PDB, ranging in size from 50 to 150 residues. These same crystal structures were used to generate decoys in a study of Rosetta@home in full structure prediction ${ }^{14}$. We used REMCGRID algorithm with 25 different parameter sets; for each parameter set, we generated 320 decoys for each of the 59 proteins by randomly choos- 
ing a residue, perturbing its phi/psi angles, and applying the best rotamer for the perturbed configuration (see Fig. 4). We computed the backbone rmsd of each decoy with respect to its crystal structure. The parameter set with the largest phi/psi perturbations and the least restricting $k T_{f}$ values resulted in the most perturbed decoy structures. We chose these decoys as our dataset because they covered the broadest range of rmsd's (including $10+\AA$ ) and showed uniform broadness across protein size. These characteristics (uniformity and broadness) are important for the proper performance of machine learning algorithms.

\section{Classification of Decoys and Features Used in SVM Training}

The decoys were then grouped into four classes based on backbone rmsd from the crystal structure, with classes $A, B, C$, and D corresponding to rmsd's of $0-1,1-3,3-10$, and $10+\AA$. For every decoy, we computed four features: (i) all-atom energy, (ii) volume, (iii) surface area, and (iv) CG-score as described above.

\section{Support Vector Machine Training}

We trained our support vector machine classifier to predict the class of each decoy using three different feature sets: (1) with all-atom energy as the sole feature, (2) with CG-score as the sole feature, and (3) with all four features used simultaneously (CG-score, all-atom energy, surface area, and volume). Table 3 and Fig. 5 show the distribution of correct predictions for the four different classes. Fig. 5A shows the distribution of classified decoys that are 0-1 $\AA$ backbone rmsd away from their native state. The "all-4" classifier predicts about $80 \%$ of the decoys correctly, whereas the classifier trained using all-atom en- 
ergy alone predicts only about $25 \%$ of the decoys correctly. This trend is consistent in all four different classes of $A, B, C$, and D. 


\section{Methods}

\section{Coarse-grained parameterization}

In the coarse-grained model, the CG-score of a protein structure is calculated by summing the pairwise energies of all the residues. The value of the sidechain-sidechain term, Ess, depends on the amino acid groups of the two residues (see Fig. 3). Five levels of energy were used to represent the different pairwise interactions: $-1,-0.5,0,+0.5$, and +1 . A positive score corresponds to an unfavorable interaction and a negative score corresponds to a favorable interaction. The five energy levels were chosen based on the biophysical characteristics of protein molecules.

\section{Computing the features of the decoys}

We computed the volume and surface area of the decoys using vossvolvox package ${ }^{15}$. The coarse-grained score was computed as described in the Results, and the all-atom energies were computed using the PHOENIX software package developed previously in the Mayo lab.

\section{Support vector machine training}

We used libsvm-3.17 open source library ${ }^{16}$ as our support vector machine-training scheme. All input and output data were scaled to -1.0 to +1.0 . We trained the classifier as a multi-class classification (C-SVC) with linear basis function kernel. We used 6-fold cross validation, and on average, generalization was around $80 \%$. 


\section{References}

1. Dill, K. A.; MacCallum, J. L. Science 2012, 338(6110), 1042-1046.

2. Summa, C. M.; Levitt, M. Proc Natl Acad Sci U S A 2007, 104(9), 3177-3182.

3. Vanommeslaeghe, K.; Hatcher, E.; Acharya, C.; Kundu, S.; Zhong, S.; Shim, J.; Darian, E.; Guvench, O.; Lopes, P.; Vorobyov, I.; Mackerell, A. D., Jr. J Comput Chem 2010, 31(4), 671-690.

4. Best, R. B.; Zhu, X.; Shim, J.; Lopes, P. E.; Mittal, J.; Feig, M.; Mackerell, A. D., Jr. J Chem Theory Comput 2012, 8(9), 3257-3273.

5. Price, D. J.; Brooks, C. L., 3rd. J Comput Chem 2002, 23(11), 1045-1057.

6. Hsieh, M. J.; Luo, R. J Phys Chem B 2010, 114(8), 2886-2893.

7. Marrink, S. J.; Risselada, H. J.; Yefimov, S.; Tieleman, D. P.; de Vries, A. H. J Phys Chem B 2007, 111(27), 7812-7824.

8. Vendruscolo, M. J Biol Phys 2001, 27(2-3), 205-215.

9. Ponder, J. W.; Case, D. A. Adv Protein Chem 2003, 66, 27-85.

10. Jewett, A. I.; Pande, V. S.; Plaxco, K. W. J Mol Biol 2003, 326(1), 247-253.

11. Tozzini, V. Curr Opin Struct Biol 2005, 15(2), 144-150.

12. Berhanu, W. M.; Jiang, P.; Hansmann, U. H. Phys Rev E Stat Nonlin Soft Matter Phys 2013, 87(1), 014701.

13. Bernstein, F. C.; Koetzle, T. F.; Williams, G. J.; Meyer, E. F., Jr.; Brice, M. D.; Rodgers, J. R.; Kennard, O.; Shimanouchi, T.; Tasumi, M. J Mol Biol 1977, 112(3), 535-542.

14. Das, R.; Qian, B.; Raman, S.; Vernon, R.; Thompson, J.; Bradley, P.; Khare, S.; Tyka, M. D.; Bhat, D.; Chivian, D.; Kim, D. E.; Sheffler, W. H.; Malmstrom, L.; Wollacott, A. M.; Wang, C.; Andre, I.; Baker, D. Proteins 2007, 69 Suppl 8, 118-128.

15. Voss, N. R.; Gerstein, M.; Steitz, T. A.; Moore, P. B. J Mol Biol 2006, 360(4), 893-906.

16. Chang, C. C.; Lin, C. J. Acm T Intel Syst Tec 2011, 2(3). 

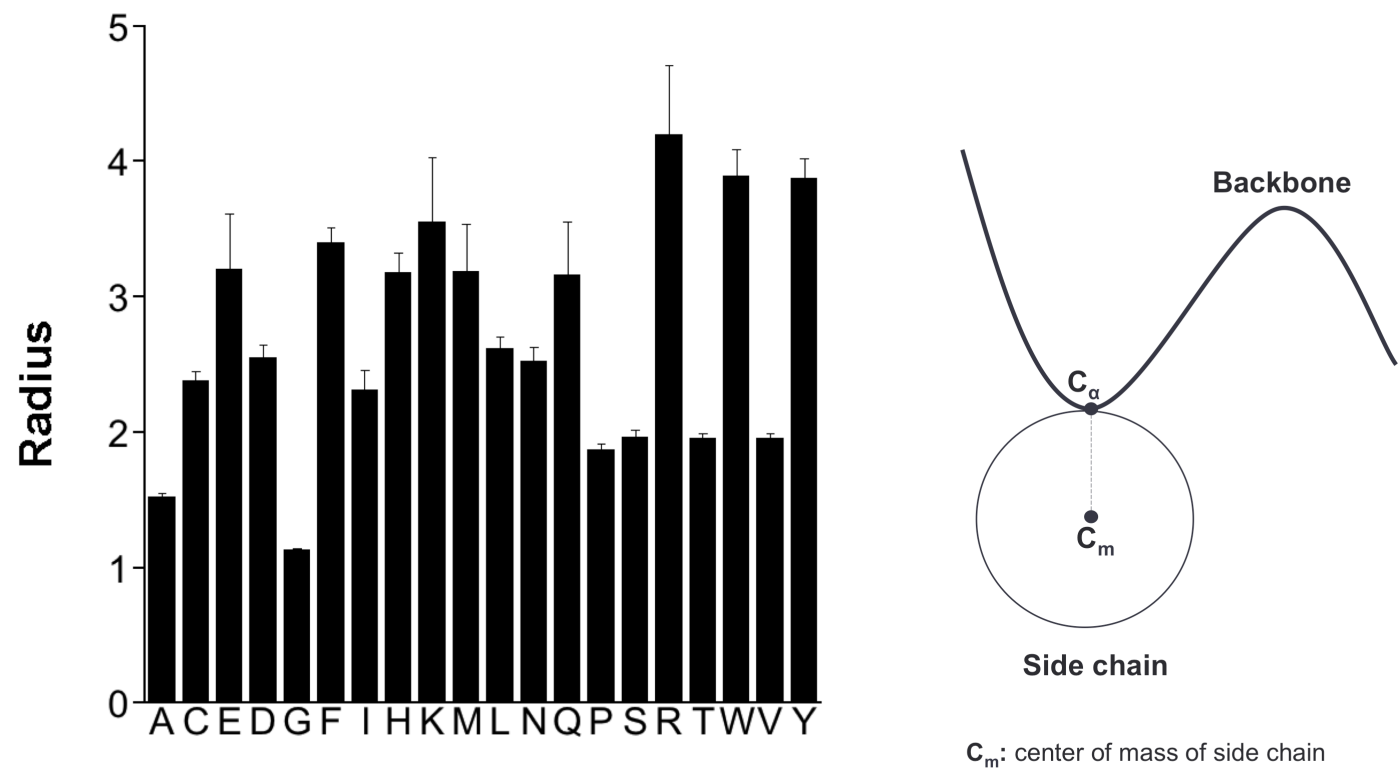

Fig 1. Coarse-grained model of protein structures; backbone is modeled as sticks connecting $C$-alpha (CA) atoms and sidechain is modeled as a sphere connected to the backbone. The average radius of each amino acid was extracted from 3,507 crystal structures from the PDB. Similarly, the average length of sticks connecting CA atoms was extracted from the same set of PDBs. 


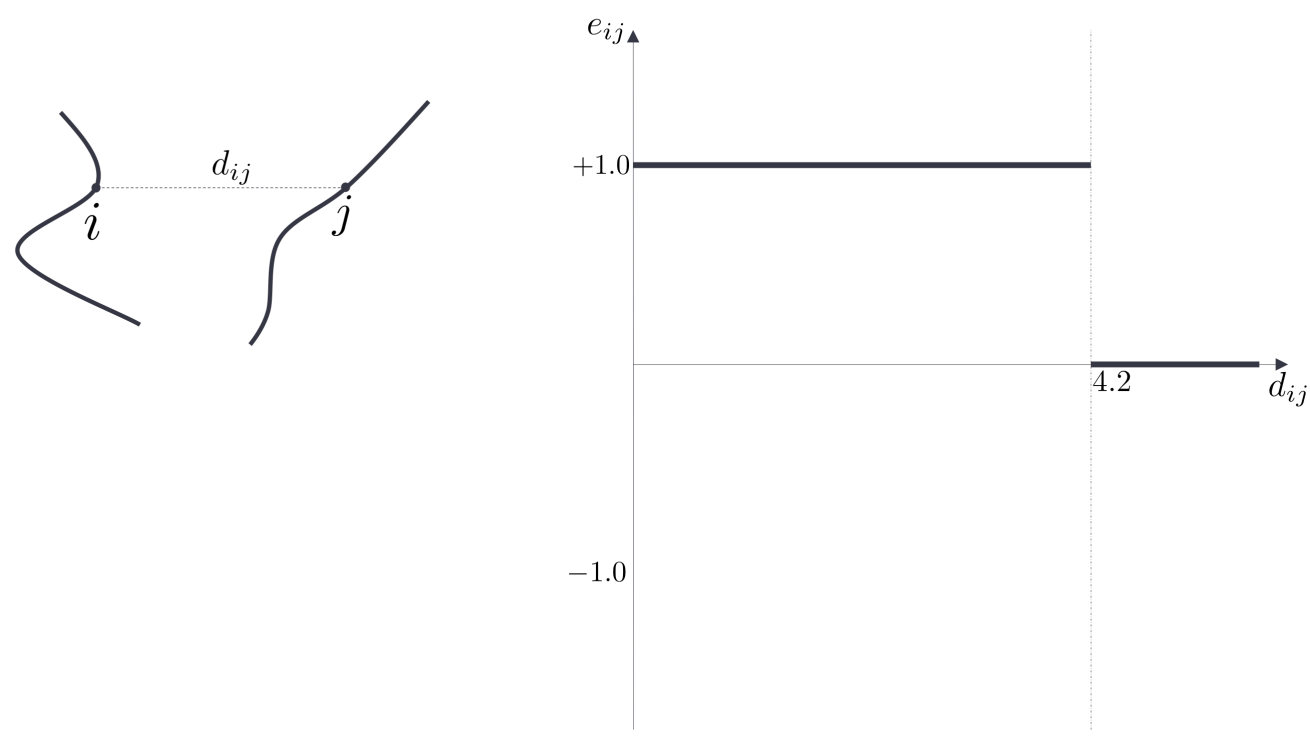

Fig 2. (A) Backbone-backbone energy, $E_{\mathrm{bb}}$; If the distance between the two CAs of residue $i$ and residue $j$ (dij) $<4.2 \AA$, a penalty of +1 is applied; at greater distances, no penalty is applied. 

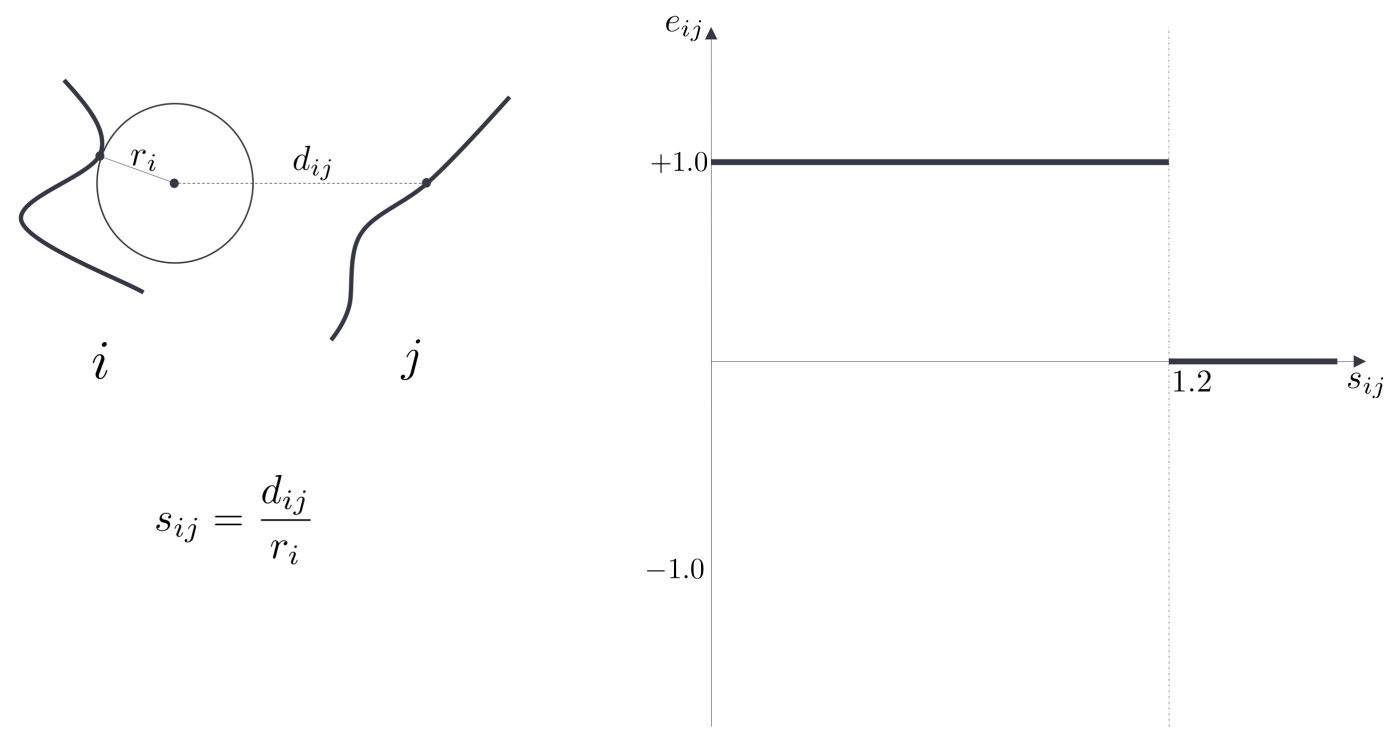

Fig 2. (B) Sidechain backbone energy term; distance between the center of mass of the sidechain of residue $i$ and the backbone of residue $j$ is denoted by $\mathrm{d}_{\mathrm{ij}}$. The effective closeness is computed by dividing the distance by radius of residue $i$. Based on closeness, there is either a penalty term or no energy contribution. If $\mathrm{s}_{\mathrm{ij}} \leq 1.2$ there is a penalty of +1 ; if $s_{i j}>1.2$ there is no penalty. 

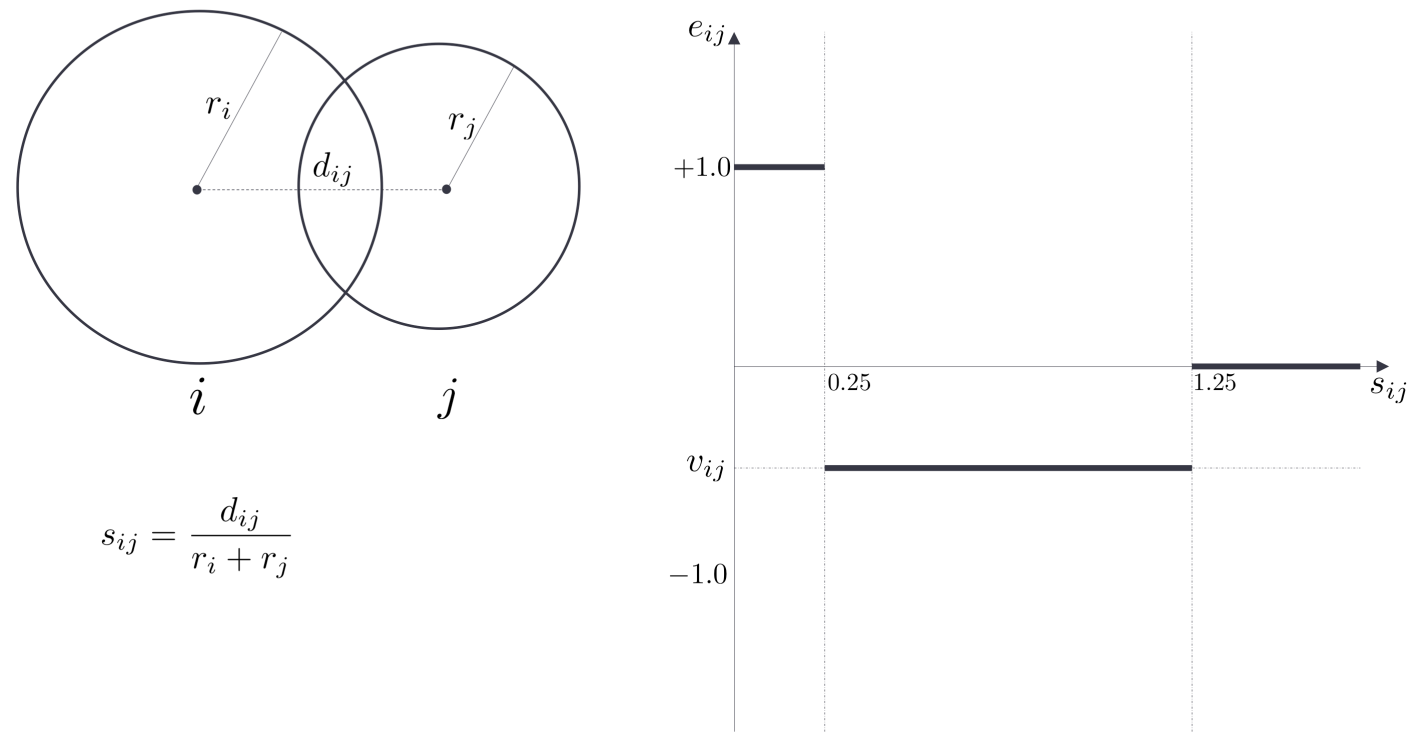

Fig 2. (C) Sidechain sidechain energy term; residue $i$ and residue $j$ are modeled as spheres. The distance between two centers of mass of the two sidechains is referred to as $d_{i j}$. Effective closeness is computed by dividing $d_{i j}$ by the sum of two radii denoted by $\mathrm{s}_{\mathrm{ij}}$. If $\mathrm{s}_{\mathrm{ij}} \geq 1.25$ there is no interaction, thus no penalty or stabilizing contribution; if $\mathrm{s}_{\mathrm{ij}} \leq 0.25$ the two sidechains are too close and it's considered a clash and there is a penalty of +1 ; if $0.25<\mathrm{s}_{\mathrm{ij}}<1.25$ the two side-chains are in contact and the corresponding stabilizing or penalty term is determined based on the amino acid groups. 


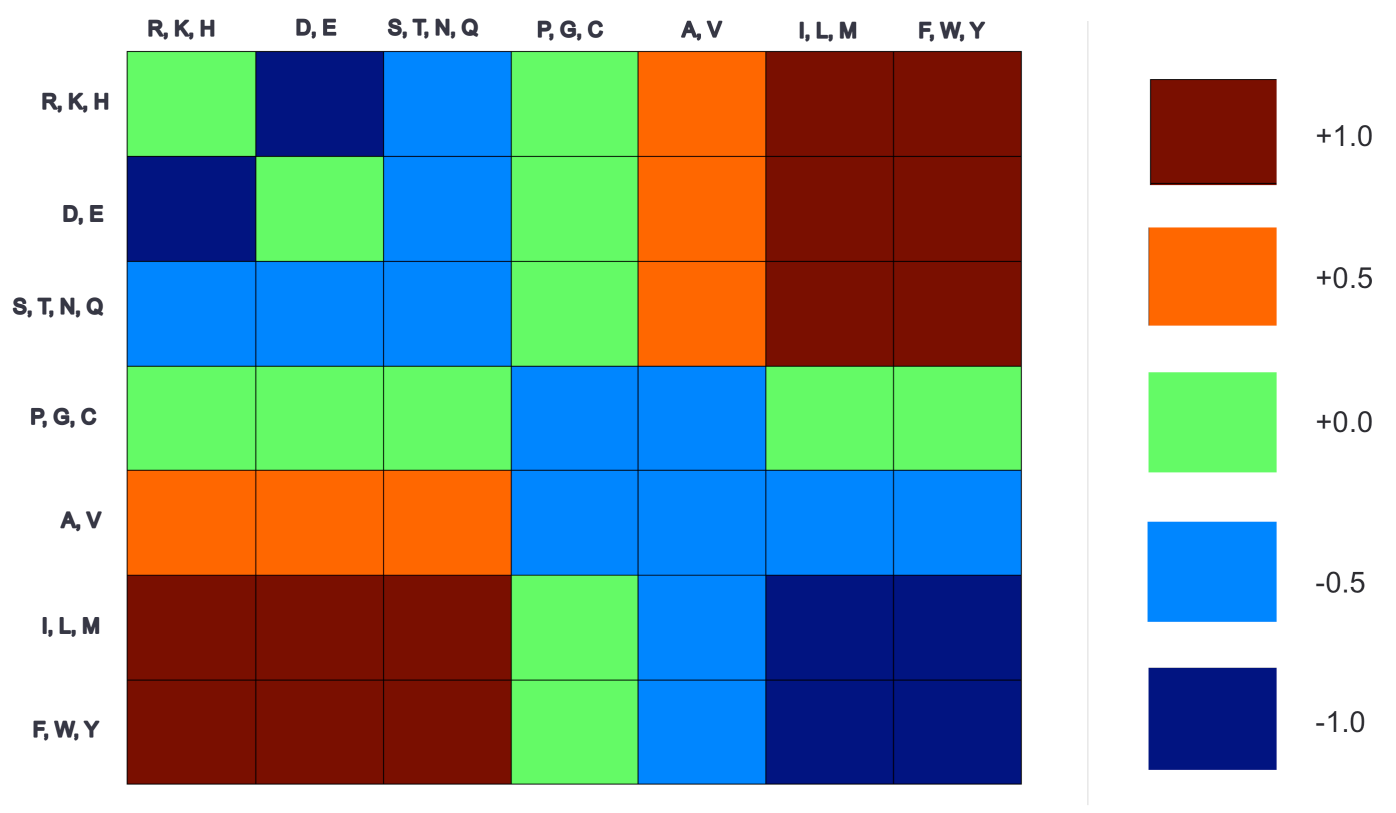

Fig 3. Pairwise energies among amino acid groups; amino acids are categorized into 7 different groups based on their biophysical properties and also their sizes. There are five energy levels: $-1,-0.5,0.0,+0.5$ and +1 for simplicity and generalization. Negative energies are stabilizing and positive energies are not stabilizing. Numbers assigned to pairwise energies are derived based on biophysical properties. 

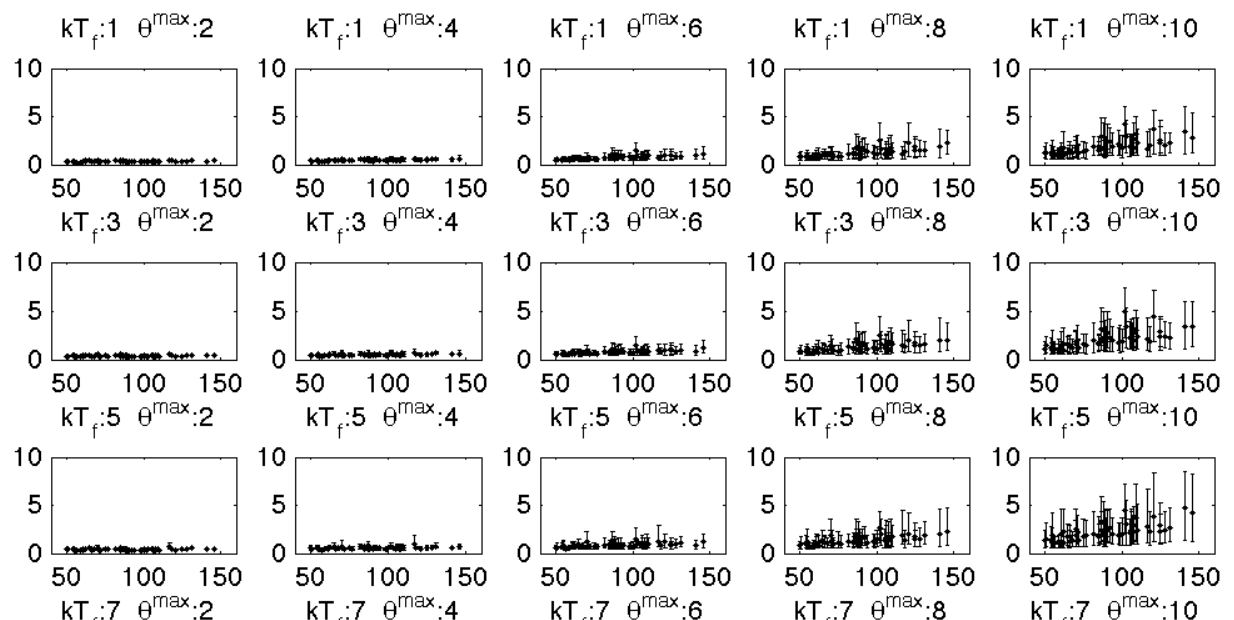

$\mathrm{kT}_{\mathrm{f}}: 5 \theta^{\max }: 10$
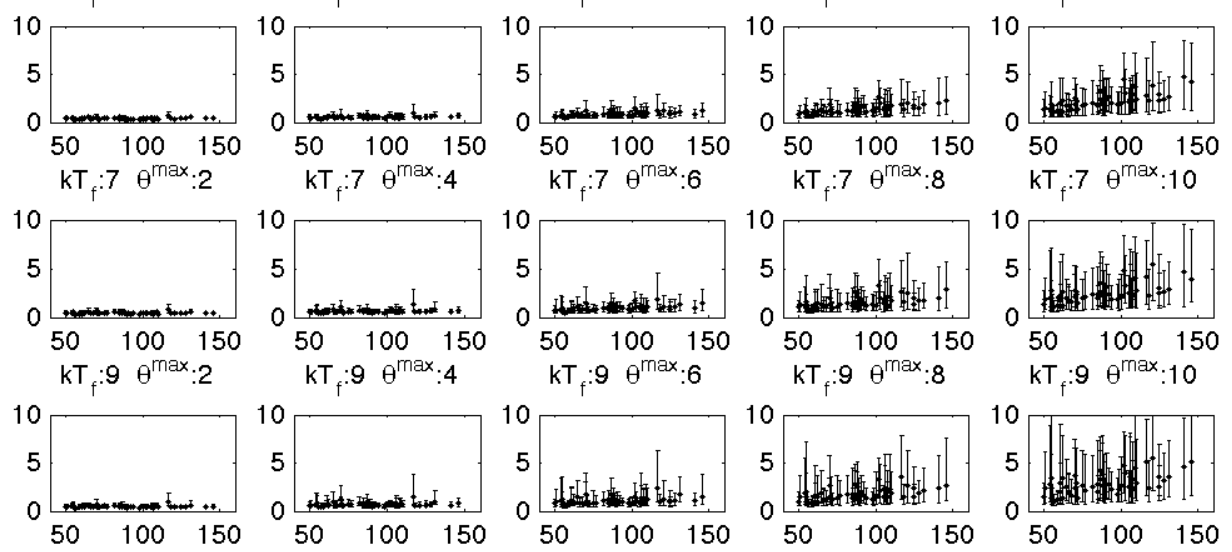

Fig 4. Backbone rmsd distribution of decoy sets generated by REMCGRID. In this figure, we have generated 25 different decoy sets by varying two parameters of the algorithm $\left(k T_{f}\right.$ and $\left.\theta\right)$. Each decoy set is depicted in a box; the $x$-axis is the size of protein in terms of number of residues, and the $y$-axis is the backbone rmsd. Each line corresponds to one single protein structure and the decoys generated for that specific structure. The line is marked by three points of 25,50 , and 75 percentiles. 

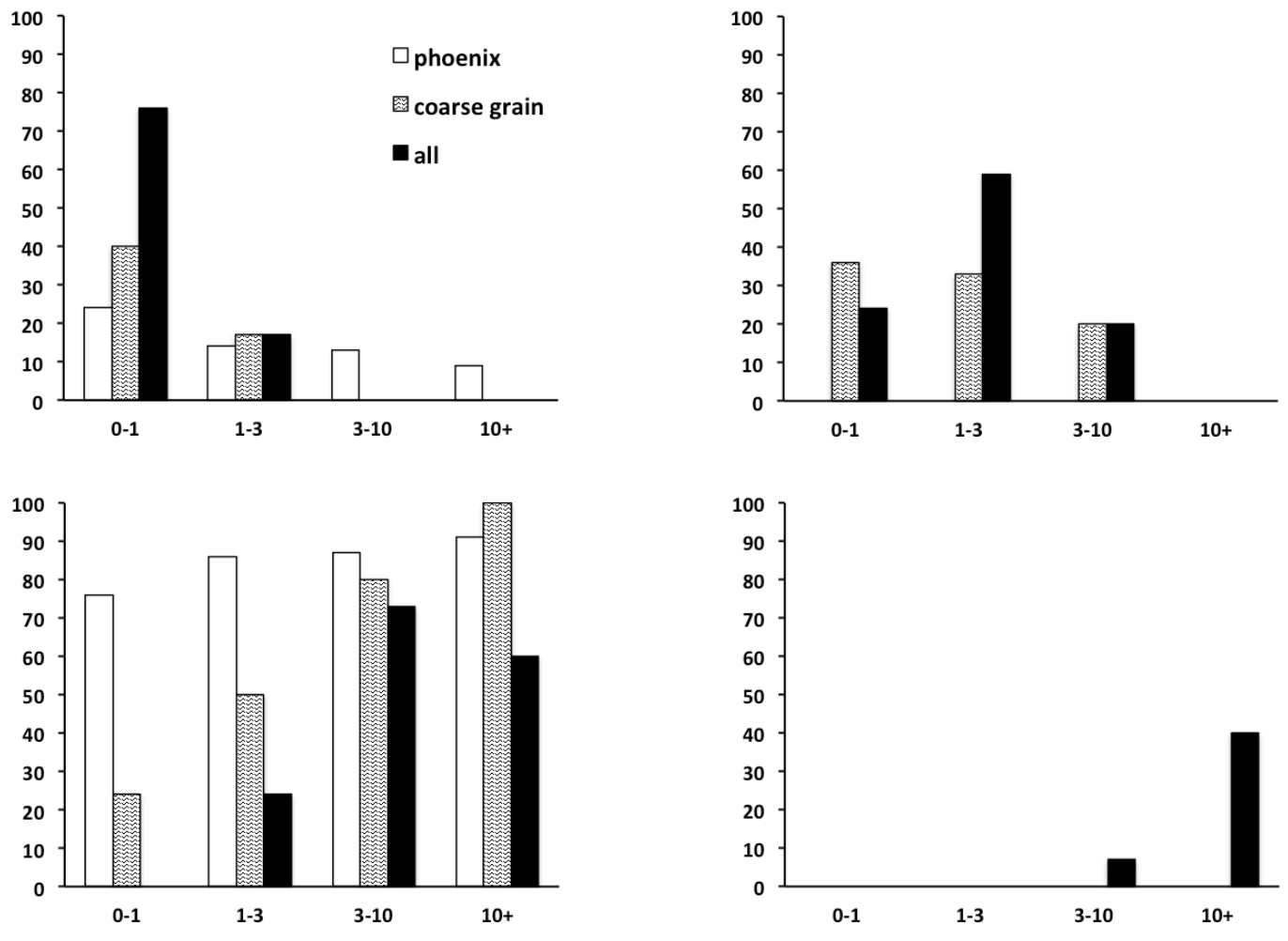

Fig 5. Comparison of three different classifiers; each classifier is trained using a different set of features, correspondingly they are trained using phoenix energy, coarse grain score, and all features composed of volume, surface area, coarse grain score and phoenix energy. (A) it shows how each of the three schemes classify decoys that are $0-1 \AA$ backbone rmsd away from their native states; $(B)$ it shows how decoys that are 1-3 $\AA$ backbone rmsd away from their native state are ranked; $(C)$ it shows ranking of decoys that are between 3-10 $\AA$ rmsd away from their native state; (D) it shows ranking of decoys that were $10+\AA$ rmsd from their native state. 


\section{PDB: $1 \mathrm{fkb}$}

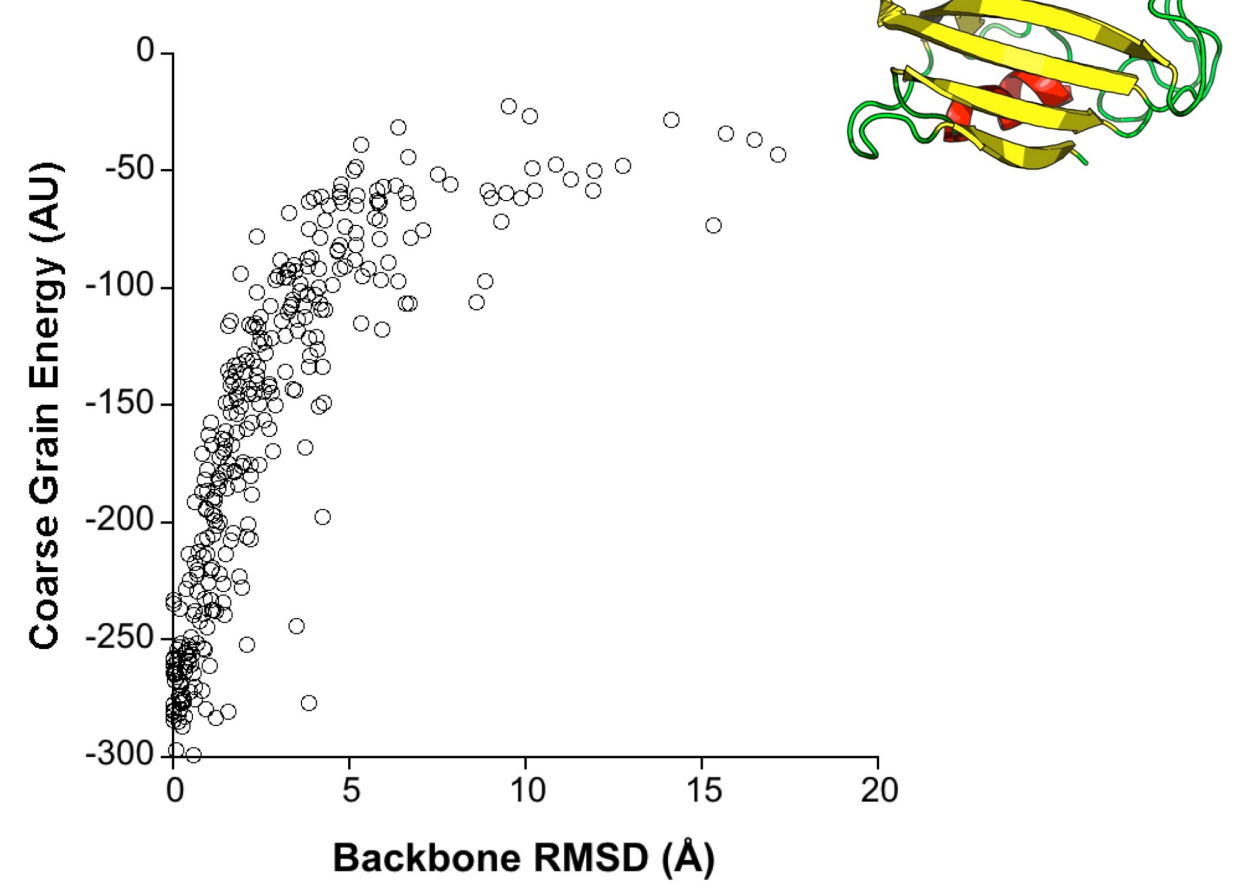

Fig S1. Coarse-grained score vs. backbone RMSD on 320 decoys generated from protein structure with pdbID 1fkb. 


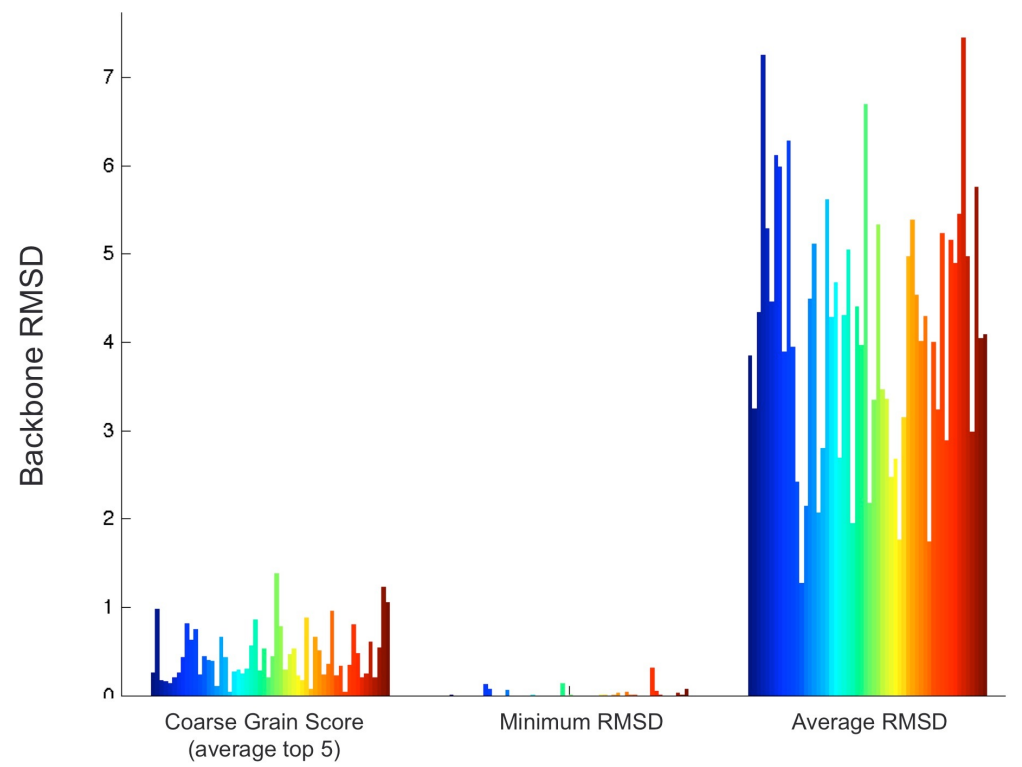

Fig S2. Comparison of coarse-grained score of structures with average RMSD and minimum RMSD in the pool of decoys, composed of 320 decoys for each of 59 protein structures; Average RMSD of top 5 structures according to coarse-grained scoring function is depicted in the first column; color coding corresponds to different proteins, ranked by number of residues. 


\section{Structure}

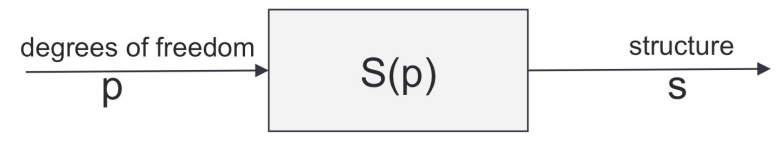

$x_{i}=x_{0}+l \sum_{k=0}^{i-1} \cos \left(\phi_{k}\right) \cos \left(\theta_{k}\right)+r_{i} \cos \left(\lambda_{i}\right) \cos \left(\gamma_{i}\right)$

$y_{i}=y_{0}+l \sum_{k=0}^{i-1} \cos \left(\phi_{k}\right) \sin \left(\theta_{k}\right)+r_{i} \cos \left(\lambda_{i}\right) \sin \left(\gamma_{i}\right)$

$z_{i}=z_{0}+l \sum_{k=0}^{i-1} \sin \left(\phi_{k}\right)+r_{i} \sin \left(\lambda_{i}\right)$

$i \in[1, n-1]$

$\phi, \lambda$ : out of plane angel

$\theta, \gamma:$ in plane angel

indexes for $\phi, \theta \rightarrow[0, n-1)$

indexes for $\lambda, \gamma \rightarrow[0, n-1]$

$l$ : length of stick between two consecutive CA atoms

Fig S3. Protein structure can be described in $p$ degrees of freedom in polar space; the above mentioned formula turns the structure from polar space into Cartesian space. 


\section{Smoothed Pairwise Energy}
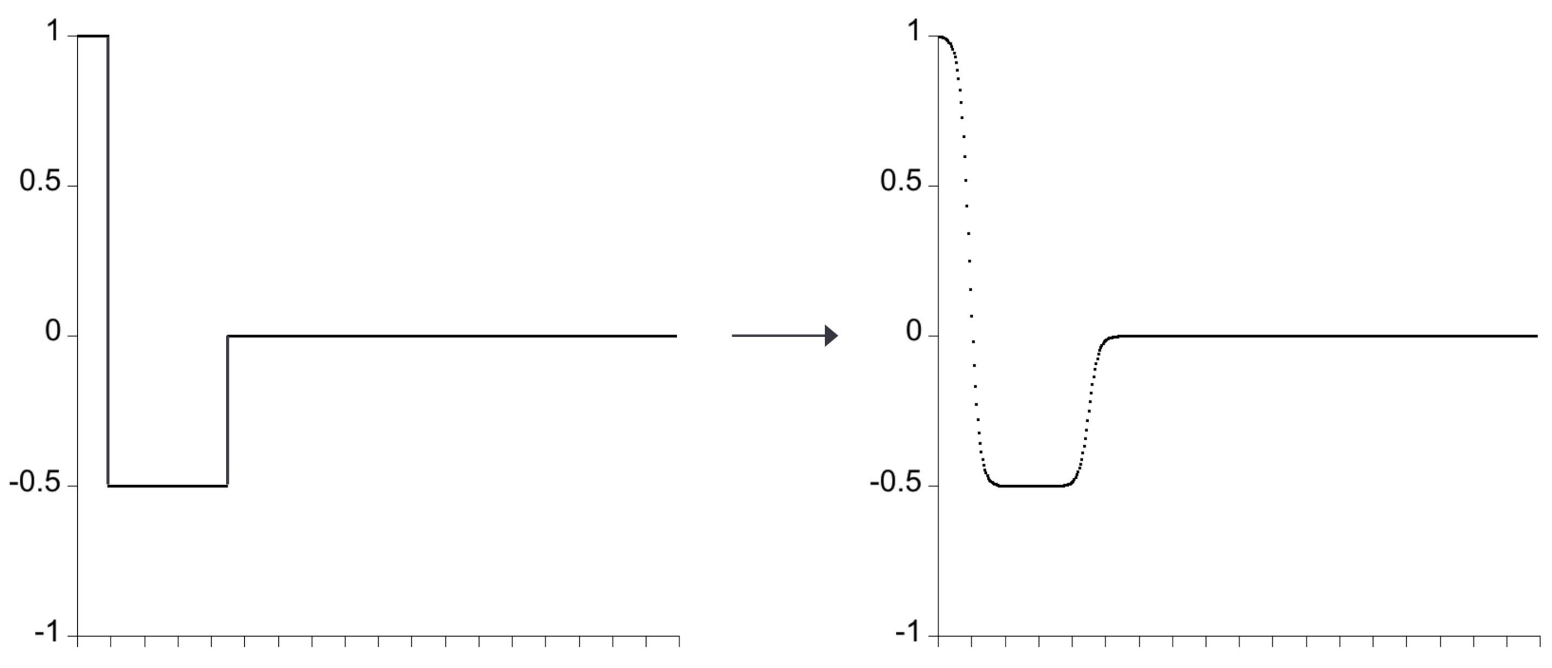

Sigmoid
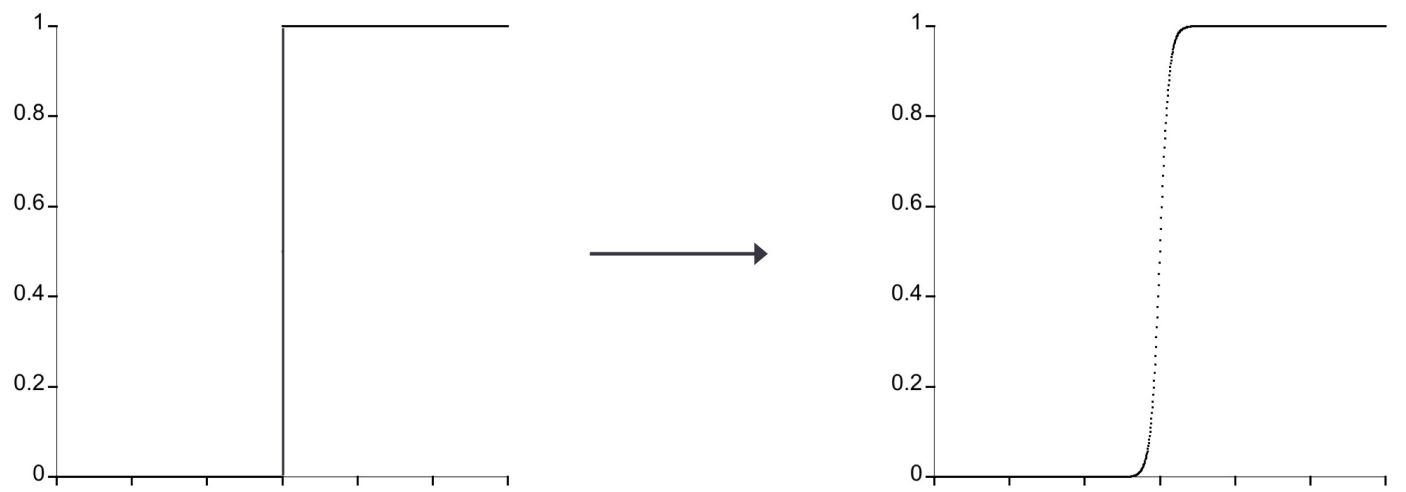

$$
y=\operatorname{step}(x-a)
$$

$y=\theta(k(x-a))=\frac{1}{1+\exp (-k(x-a))}$ 
Fig S4. Modeling the scoring function using soft sigmoid functions; major benefit of sigmoid functions over step functions is that their derivative exists and easily can be calculated, whereas in the case of step functions, the derivative is an impulse function which is not easy to work with.

\section{Energy (SS)}

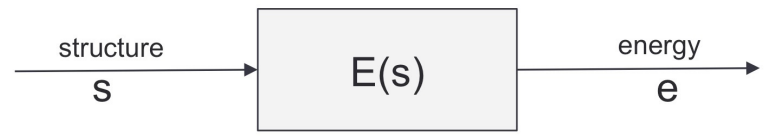

$E=\sum_{i=0}^{n-1} \sum_{j=0}^{i-1} e_{i j} \cdot \operatorname{coeff}\left(e_{i j}\right)$

$\operatorname{coeff}\left(e_{i j}\right)=(\sqrt{i-j}-1) \cdot \theta\left(-k \cdot e_{i j}\right)+1 ;$ where $k=25$

$e_{i j}=1-\left(1-v_{i j}\right) \theta\left(k\left(s_{i j}-a\right)\right)-v_{i j} \cdot \theta\left(k\left(s_{i j}-b\right)\right)$; where $k=25, a=0.0625$,

$s_{i j}=\frac{1}{\left(r_{i}+r_{j}\right)^{2}}\left[\Delta x_{i j}^{2}+\Delta y_{i j}^{2}+\Delta z_{i j}^{2}\right]$

$b=1.5625$

where:

$$
\begin{aligned}
\Delta x_{i j} & =\left(l \sum_{k=j}^{i-1} \cos \left(\phi_{k}\right) \cos \left(\theta_{k}\right)+r_{i} \cos \left(\lambda_{i}\right) \cos \left(\gamma_{i}\right)-r_{j} \cos \left(\lambda_{j}\right) \cos \left(\gamma_{j}\right)\right) \\
\Delta y_{i j} & =\left(l \sum_{k=j}^{i-1} \cos \left(\phi_{k}\right) \sin \left(\theta_{k}\right)+r_{i} \cos \left(\lambda_{i}\right) \sin \left(\gamma_{i}\right)-r_{j} \cos \left(\lambda_{j}\right) \sin \left(\gamma_{j}\right)\right) \\
\Delta z_{i j} & =\left(l \sum_{k=j}^{i-1} \sin \left(\phi_{k}\right)+r_{i} \sin \left(\lambda_{i}\right)-r_{j} \sin \left(\lambda_{j}\right)\right)
\end{aligned}
$$

Fig S5. Sidechain-sidechain energy term calculated based on Cartesian coordinates in the structure. 


\section{Energy (BB)}

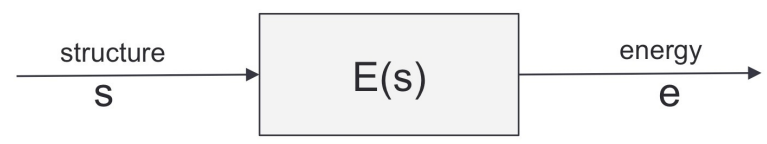

$$
\begin{aligned}
& \Delta x_{i j}^{b b}=l \sum_{k=j}^{i-1} \cos \left(\phi_{k}\right) \cos \left(\theta_{k}\right) \\
& \Delta y_{i j}^{b b}=l \sum_{k=j}^{i-1} \cos \left(\phi_{k}\right) \sin \left(\theta_{k}\right) \\
& \Delta z_{i j}^{b b}=l \sum_{k=j}^{i-1} \sin \left(\phi_{k}\right) \\
& d_{i j}^{b b^{2}}=\Delta x_{i j}^{b b^{2}}+\Delta y_{i j}^{b b^{2}}+\Delta z_{i j}^{b b^{2}} \\
& E^{b b}=\sum_{i=0}^{n-1} \sum_{j=0}^{i-1} e_{i j}^{b b} \\
& e_{i j}^{b b}=\theta\left(-k^{b b}\left(d_{i j}^{b b^{2}}-a^{b b^{2}}\right)\right) ; \text { where } a^{b b}=3.25 \text { and } k^{b b}=3
\end{aligned}
$$

Fig S6. Backbone-backbone energy term calculated based on Cartesian coordinates in the structure. 


\section{Energy (SB)

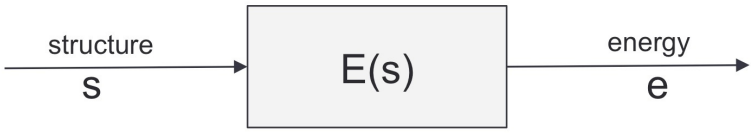

for $i>j$ and

$$
\begin{aligned}
& \Delta x_{i j}^{s b}=l \sum_{k=j}^{i-1} \cos \left(\phi_{k}\right) \cos \left(\theta_{k}\right)+r_{i} \cos \left(\lambda_{i}\right) \cos \left(\gamma_{i}\right) \\
& \Delta y_{i j}^{s b}=l \sum_{k=j}^{i-1} \cos \left(\phi_{k}\right) \sin \left(\theta_{k}\right)+r_{i} \cos \left(\lambda_{i}\right) \sin \left(\gamma_{i}\right) \\
& \Delta z_{i j}^{s b}=l \sum_{k=j}^{i-1} \sin \left(\phi_{k}\right)+r_{i} \sin \left(\lambda_{i}\right)
\end{aligned}
$$

for $j>i$

$$
\begin{aligned}
& \Delta x_{i j}^{s b}=-l \sum_{k=i}^{j-1} \cos \left(\phi_{k}\right) \cos \left(\theta_{k}\right)+r_{i} \cos \left(\lambda_{i}\right) \cos \left(\gamma_{i}\right) \\
& \Delta y_{i j}^{s b}=-l \sum_{k=i}^{j-1} \cos \left(\phi_{k}\right) \sin \left(\theta_{k}\right)+r_{i} \cos \left(\lambda_{i}\right) \sin \left(\gamma_{i}\right) \\
& \Delta z_{i j}^{s b}=-l \sum_{k=i}^{j-1} \sin \left(\phi_{k}\right)+r_{i} \sin \left(\lambda_{i}\right) \\
& s_{i j}^{s b}=\frac{1}{r_{i}^{2}}\left[\Delta x_{i j}^{s b^{2}}+\Delta y_{i j}^{s b^{2}}+\Delta z_{i j}^{s b^{2}}\right] \\
& E^{s b}=\sum_{i=0}^{n-1} \sum_{j=0}^{n-1} e_{i j}^{s b} ; \text { where } e_{i i}^{s b}=0 \\
& e_{i j}^{s b}=\theta\left(-k^{s b}\left(s_{i j}^{s b}-a^{s b^{2}}\right)\right) ; \text { where } a^{s b}=1.05 \text { and } k^{s b}=25
\end{aligned}
$$

Fig S7. Sidechain-backbone energy term calculated based on Cartesian coordinates in the structure. 


\section{Gradient}

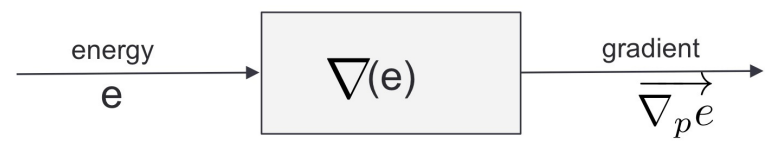

$$
\begin{aligned}
p^{r} & =\left(\phi_{0}^{r}, \theta_{0}^{r}, \lambda_{0}^{r}, \gamma_{0}^{r}, \ldots, \phi_{n-2}^{r}, \theta_{n-2}^{r}, \lambda_{n-2}^{r}, \gamma_{n-2}^{r}, \lambda_{n-1}^{r}, \gamma_{n-1}^{r}\right) \\
p^{r+1} & =p^{r}-\epsilon \nabla E\left(p^{r}\right) \\
\frac{\partial E}{\partial \phi_{m}} & =\sum_{i=0}^{n-1} \sum_{j=0}^{i-1} \frac{\partial e_{i j}}{\partial \phi_{m}}\left[(\sqrt{i-j}-1) \theta\left(-k e_{i j}\right)\left(1-k \theta\left(k e_{i j}\right) e_{i j}\right)+1\right] \\
\frac{\partial E}{\partial \phi_{m}} & =\sum_{i=0}^{n-1} \sum_{j=0}^{i-1} \frac{\partial e_{i j}}{\partial s_{i j}} \cdot \frac{\partial s_{i j}}{\partial \phi_{m}}\left[(\sqrt{i-j}-1) \theta\left(-k e_{i j}\right)\left(1-k \theta\left(k e_{i j}\right) e_{i j}\right)+1\right] \\
\frac{\partial e_{i j}}{\partial s_{i j}} & =-k\left[\left(1-v_{i j}\right) \theta\left(k\left(s_{i j}-a\right)\right) \theta\left(-k\left(s_{i j}-a\right)\right)+v_{i j} \theta\left(k\left(s_{i j}-b\right)\right) \theta\left(-k\left(s_{i j}-b\right)\right)\right] \\
\frac{\partial s_{i j}}{\partial \phi_{m}} & =\frac{2}{\left(r_{i}+r_{j}\right)^{2}}\left[\Delta x_{i j} \cdot \frac{\partial \Delta x_{i j}}{\partial \phi_{m}}+\Delta y_{i j} \cdot \frac{\partial \Delta y_{i j}}{\partial \phi_{m}}+\Delta z_{i j} \cdot \frac{\partial \Delta z_{i j}}{\partial \phi_{m}}\right] \\
\frac{\partial \Delta x_{i j}}{\partial \phi_{m}} & =-l \cdot \sin \left(\phi_{m}\right) \cos \left(\theta_{m}\right) \\
\frac{\partial \Delta y_{i j}}{\partial \phi_{m}} & =-l \cdot \sin \left(\phi_{m}\right) \sin \left(\theta_{m}\right) \\
\frac{\partial \Delta z_{i j}}{\partial \phi_{m}} & =l \cdot \cos \left(\theta_{m}\right)
\end{aligned}
$$

Fig S8. Gradient of the energy can be directly calculated from the energy (score function). It can be used to explore the energy landscape and minimize the score and find the lowest energy structure. 

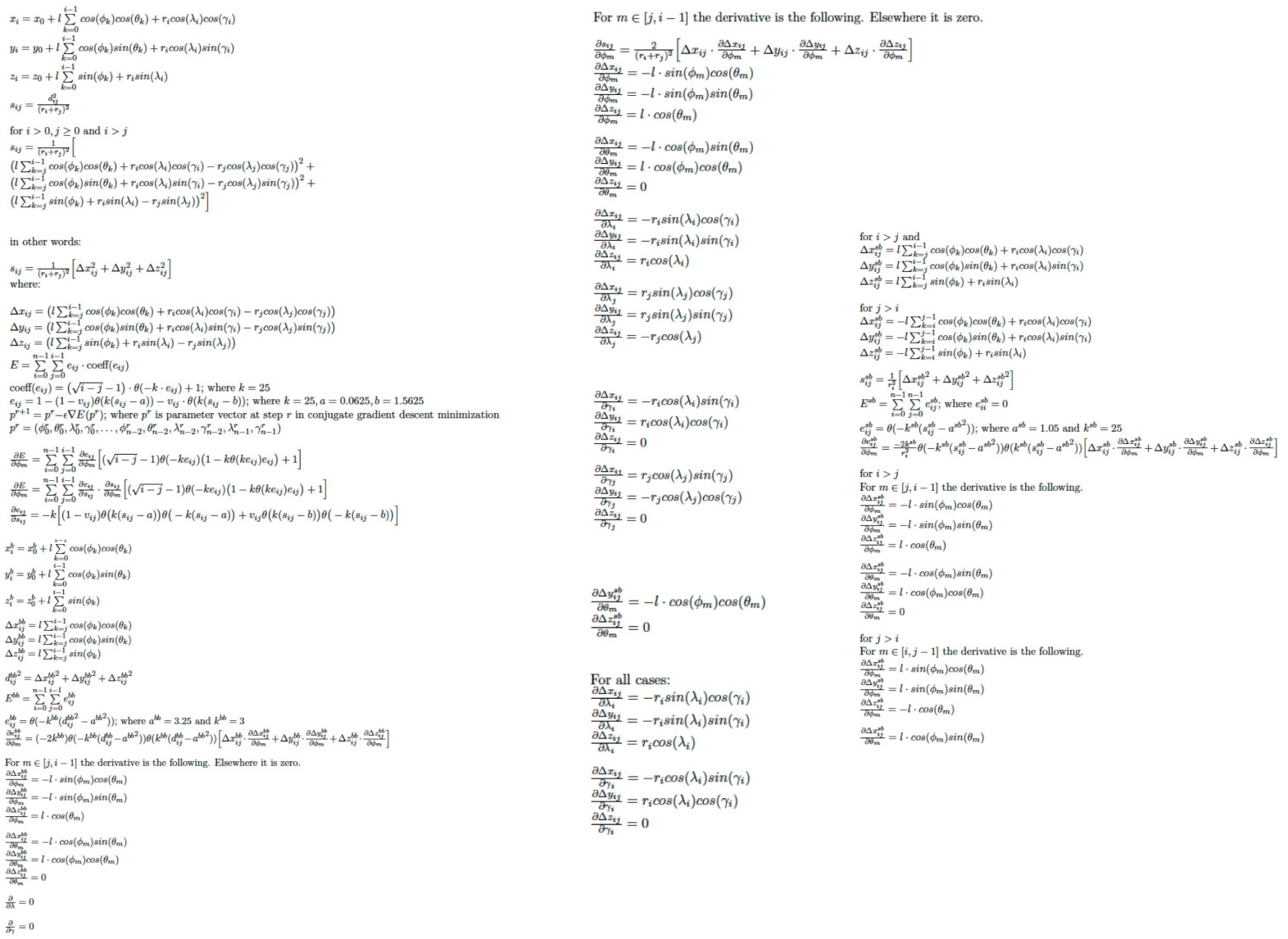

Fig S9. Coarse-grained model and scoring function and gradient of the score. 


\section{Size Distribution}

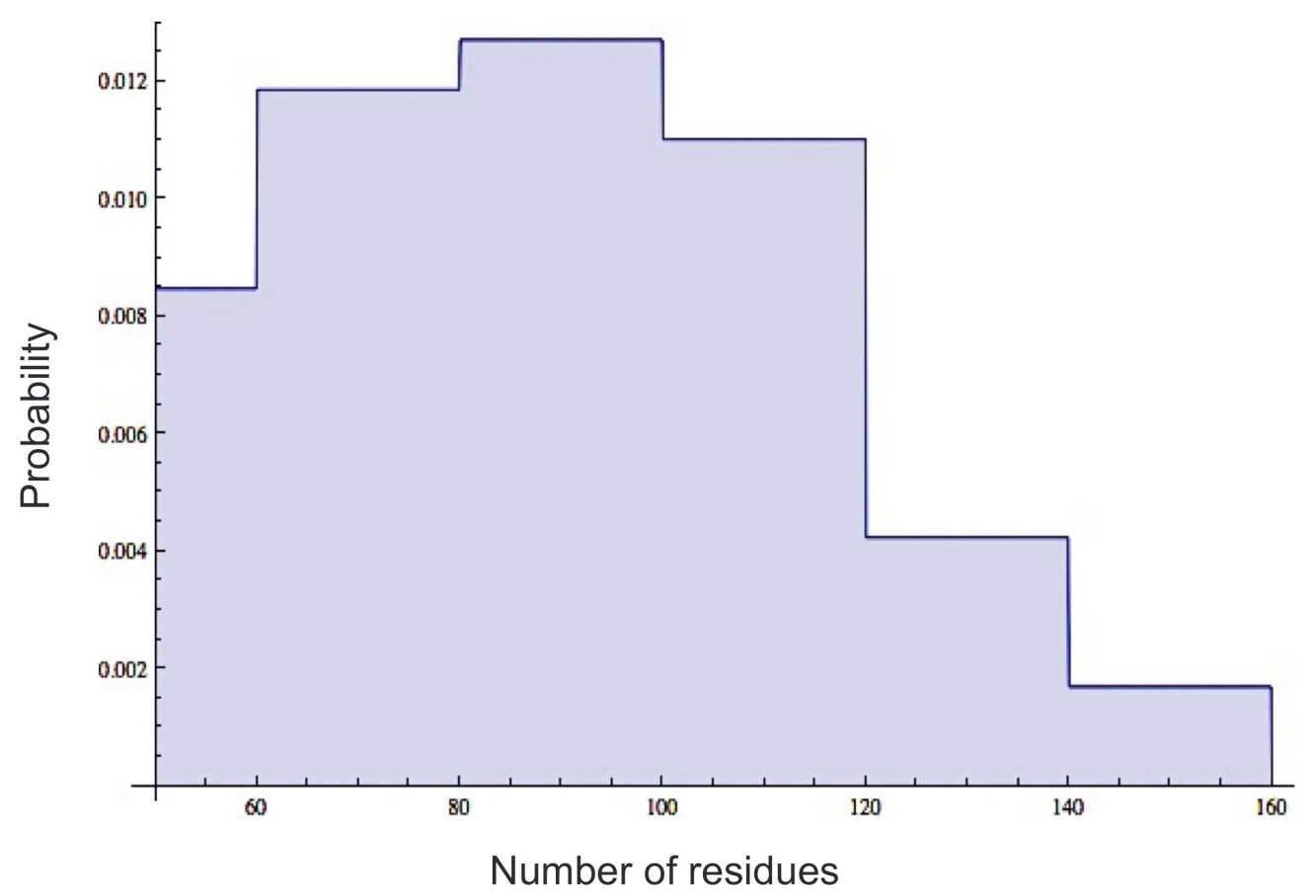

Fig S10. Size(number of amino acids) distribution of 59 high-resolution protein structures used to generate decoys. 


\begin{tabular}{|lll|}
\hline Group & Amino Acids & Description \\
\hline $\mathbf{1}$ & R, K, H & Positive \\
$\mathbf{2}$ & D, E & Negative \\
$\mathbf{3}$ & S, T, N, Q & Polar uncharged \\
$\mathbf{5}$ & P, G, C & Special \\
$\mathbf{6}$ & A, V & Small hydrophobic \\
$\mathbf{7}$ & I, L, M & Large hydrophobic \\
\hline
\end{tabular}

Table 1. Amino acids are categorized into seven different groups based on their size and biochemical properties. 


\begin{tabular}{|l|lllllll|}
\hline & $\mathbf{1}$ & $\mathbf{2}$ & $\mathbf{3}$ & $\mathbf{4}$ & $\mathbf{5}$ & $\mathbf{6}$ & $\mathbf{7}$ \\
\hline $\mathbf{1}$ & +0.0 & -1.0 & -0.5 & +0.0 & +0.5 & +1.0 & +1.0 \\
$\mathbf{2}$ & -1.0 & +0.0 & -0.5 & +0.0 & +0.5 & +1.0 & +1.0 \\
$\mathbf{4}$ & -0.5 & -0.5 & -0.5 & +0.0 & +0.5 & +1.0 & +1.0 \\
$\mathbf{5}$ & +0.0 & +0.0 & +0.0 & -0.5 & -0.5 & +0.0 & +0.0 \\
$\mathbf{6}$ & +0.5 & +0.5 & +0.5 & -0.5 & -0.5 & -0.5 & -0.5 \\
$\mathbf{7}$ & +1.0 & +1.0 & +1.0 & +0.0 & -0.5 & -1.0 & -1.0 \\
\hline
\end{tabular}

Table 2. Pairwise energy for residue $i$ and $j$ based on their amino acid group. Amino acids are categorized based on their biophysical properties. The energy corresponding two amino acids is given in this table. There are five energy levels for simplicity. 


\begin{tabular}{|l|l|lllll|}
\hline (a) Phoenix energy & \multicolumn{5}{|c|}{ Actual class } \\
\hline & & A & B & C & D \\
\cline { 2 - 6 } Predicted class (\%) & A & 24 & 14 & 13 & 9 \\
& B & 0 & 0 & 0 & 0 \\
& C & 76 & 86 & 87 & 91 \\
& D & 0 & 0 & 0 & 0 \\
\hline
\end{tabular}

\begin{tabular}{|l|l|llll|}
\hline (b) Coarse grain & \multicolumn{5}{|c|}{ Actual class } \\
\hline & & A & B & C & D \\
\cline { 2 - 6 } Predicted class (\%) & A & 40 & 17 & 0 & 0 \\
& B & 36 & 33 & 20 & 0 \\
& C & 24 & 50 & 80 & 100 \\
& D & 0 & 0 & 0 & 0 \\
\hline
\end{tabular}

\begin{tabular}{|c|c|c|c|c|c|}
\hline (c) All features & \multicolumn{5}{|c|}{ Actual class } \\
\hline \multirow{5}{*}{ Predicted class (\%) } & & A & B & C & D \\
\hline & A & 76 & 17 & 0 & 0 \\
\hline & B & 24 & 59 & 20 & 0 \\
\hline & C & 0 & 24 & 73 & 60 \\
\hline & D & 0 & 0 & 7 & 40 \\
\hline
\end{tabular}

Table 3. Comparison of phoenix energy, coarse grain score, and all features in training the support vector machine in classifying decoys. Every individual decoy is classified into one of the four classes of A, B, C, and D that corresponds to $0-1,1-3,3-10,10+$ backbone rmsds. In all of the tables we demonstrate predicted class of decoys against the actual class of a decoy. Each column presents the distribution of decoys predicted in each of four classes. For instance, in table (a), $76 \%$ of decoys that belonged to class A were predicted to belong to class C (wrong prediction). As another example, in table (c), $59 \%$ of decoys that belonged to class B were predicted to belong to class B (correct prediction). 
Appendix 


\section{Appendix A}

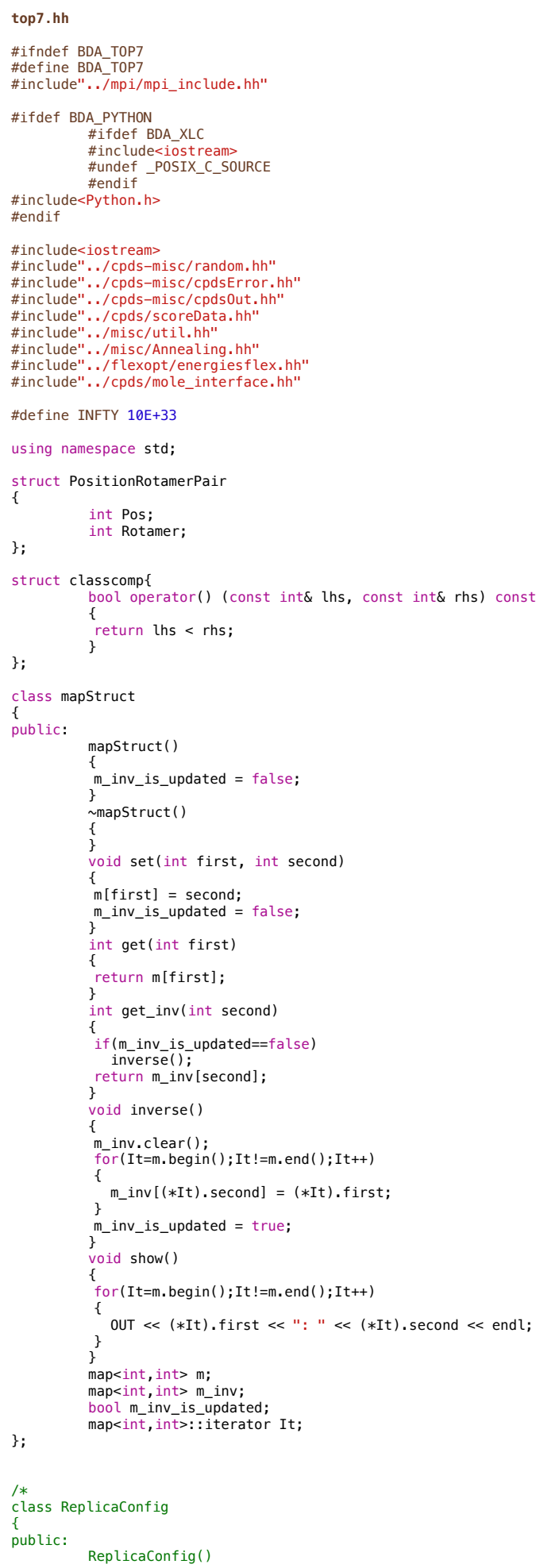




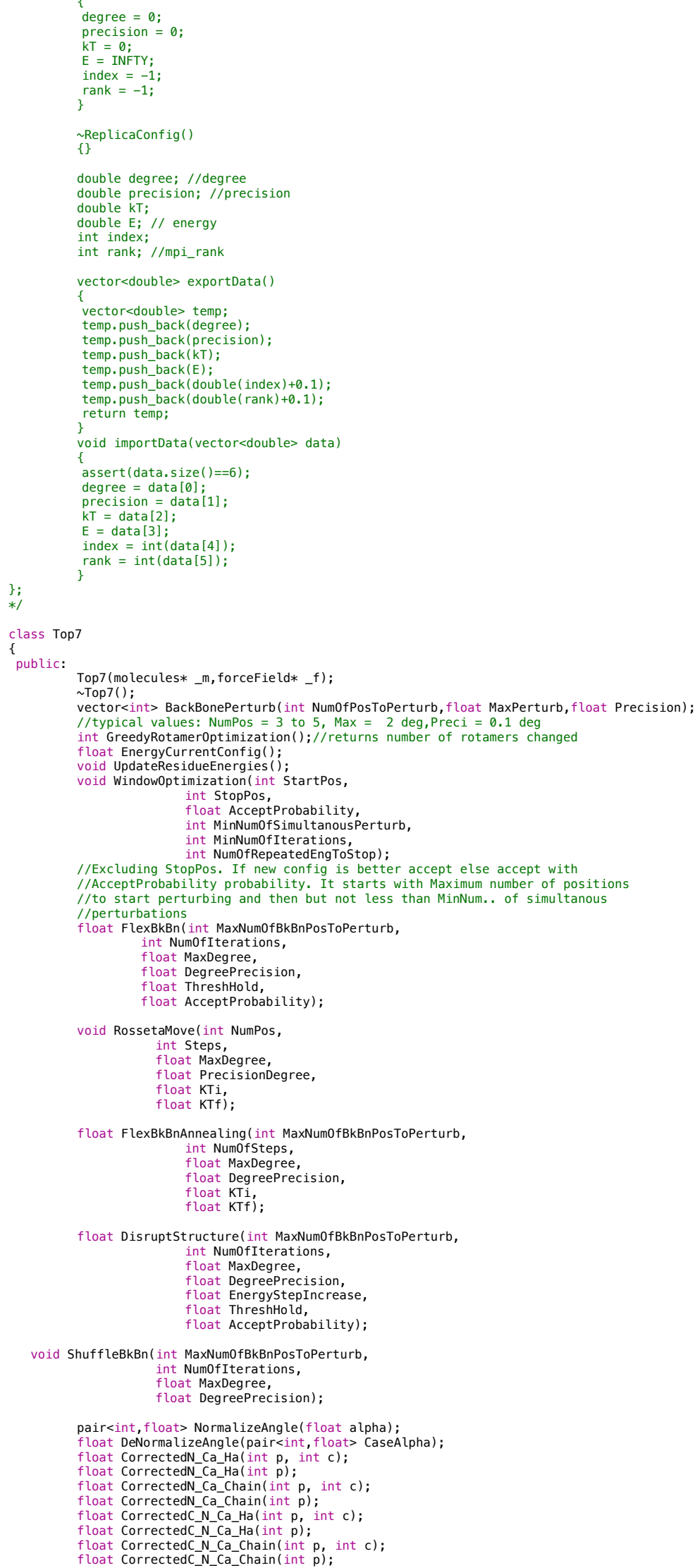

void ShuffleBkBn (int MaxNum0fBkBnPosToPerturb, int NumOfIterations, float MaxDegree,

float DegreePrecision)

pair<int, float> NormalizeAngle ( $f$ loat alpha)

pair<int, float> NormalizeAngle(float alpha);
float DeNormalizeAngle(pair<int, float> CaseAlpha);

float CorrectedN_Ca_Ha(int p, int c);

float CorrectedN_Ca_Ha (int p, int
float CorrectedN_Ca_Ha(int p);

float CorrectedN_Ca_Chain (int $p$, int $c$ )

float CorrectedN_CaChain(int p)

float CorrectedC $N$ Ca Ha (int $p$, int c),

float CorrectedC $\mathrm{NCa}^{-} \mathrm{Ha}$ (int p).

float CorrectedC N Ca Chain (int p, int c);

float CorrectedC_NCCa_Chain(int p). 


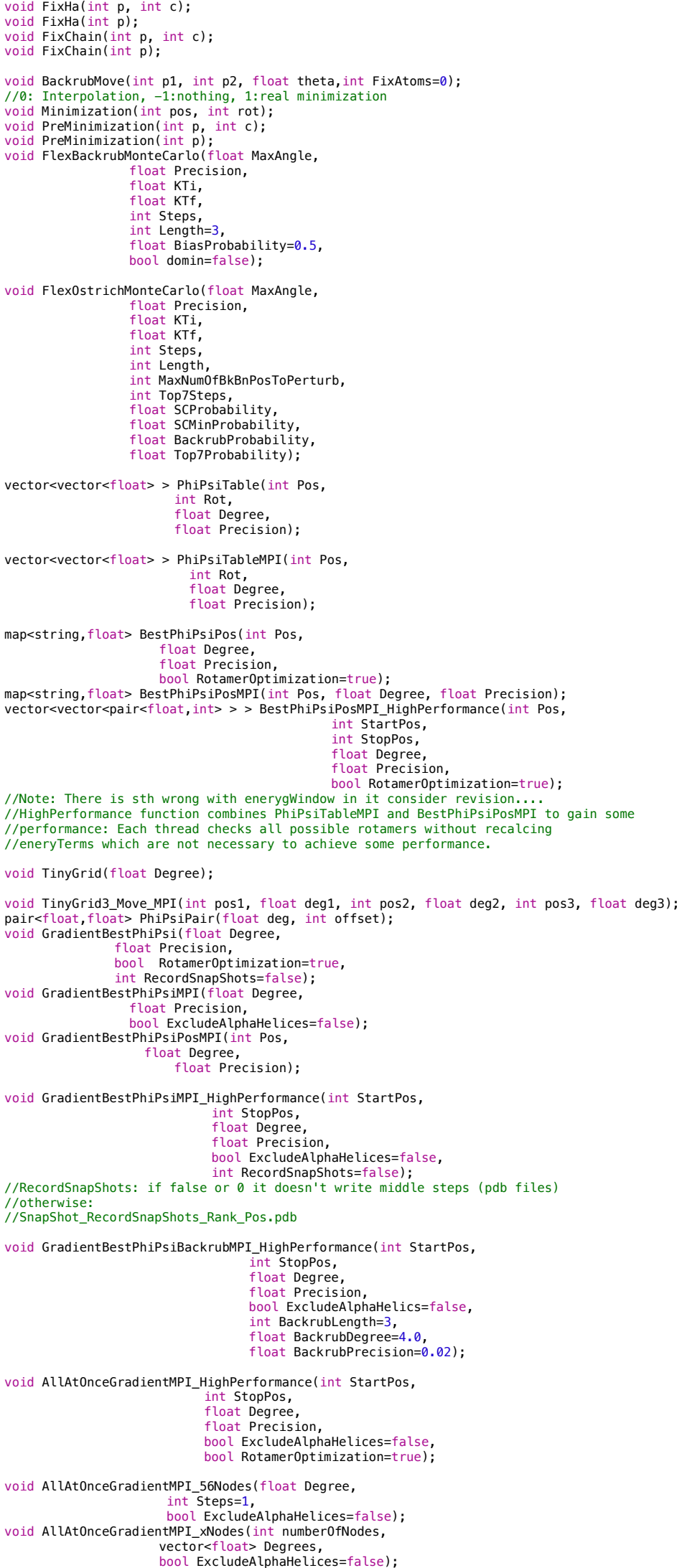




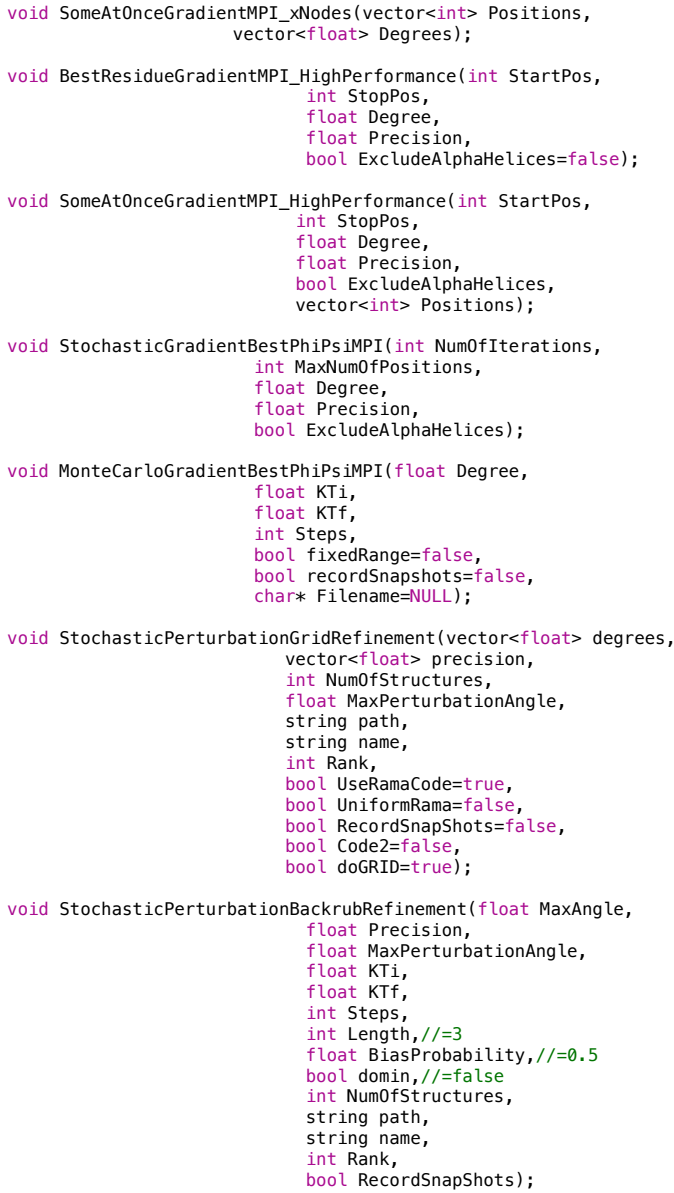

void StochasticRamaPerturbationDecoyGeneration (int Num0fStructures,

$$
\begin{aligned}
& \text { string path, } \\
& \text { string name, }
\end{aligned}
$$

float PerturbationRate $=0.1$ ) dorible double precision, size $t$ steps,

void ReplicaExchangeMonteCarloGRID(vector<float> degrees, vector<float> precisions,

int rounds,

int steps_sync,

int round=1):

void SimulatedAnnealingReplicaExchangeMonteCarloGRID ( float kT_high_i, float kT_high_f,

float $k T_{-}$low $-\dot{i}$,

float degree_scale,

int rounds,

int rounds_of_rounds,

int steps_sync);

void StochasticPDBFragmentRefinement();

void SnapShotRecorder(int SnapShot, int Pos);

vector<float> PhiPsiRepresentation( );

void GreedyMinimization ( );

bool ByPassRank(float MaxDegree, float DegreePrecision); map<string, float> CorrelationTwoPos (int Pos1, int Pos2)

void RotamerPerturb(int Position):

tion (float MaxAngle, bool Sequential=true); void RamaStructurePerturbation (bool Uniform=false, bool Code2=false,

//PerturbationRate is probability of a position to be perturbed

vector $<$ vector $<$ int $>>$ GetIntMatrix (string filename);

vector $<$ vector $<$ float $>>$ GetFloatMatrix (string filename);

template $<$ class $T>$ pair $<$, pair $<$ int, int $>>\min ($ vector $<$ vector $<T>>v e c$ ):

//Walks through the structure residue by residue and perturbs each phi angle 


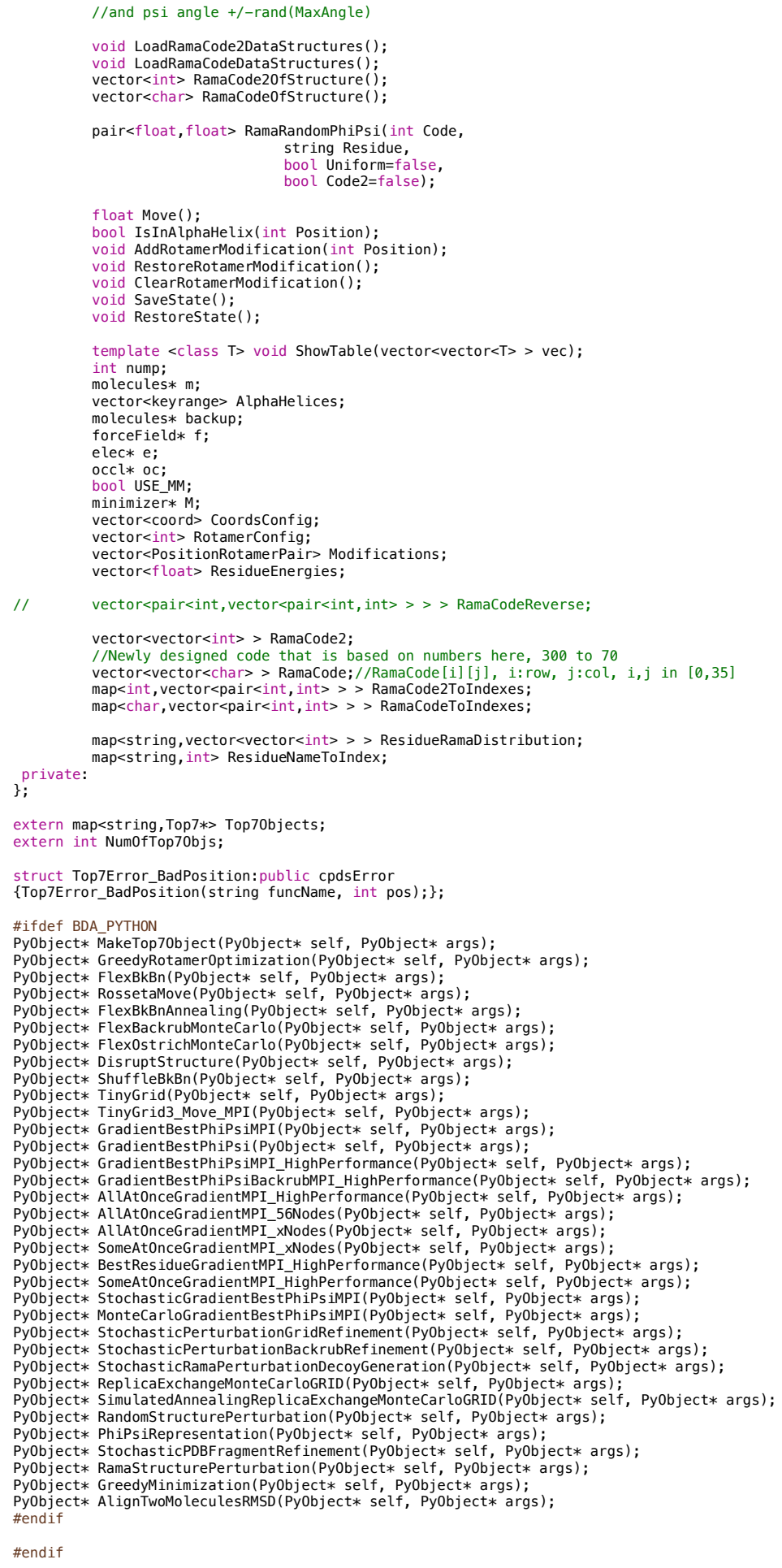




\section{Appendix B}

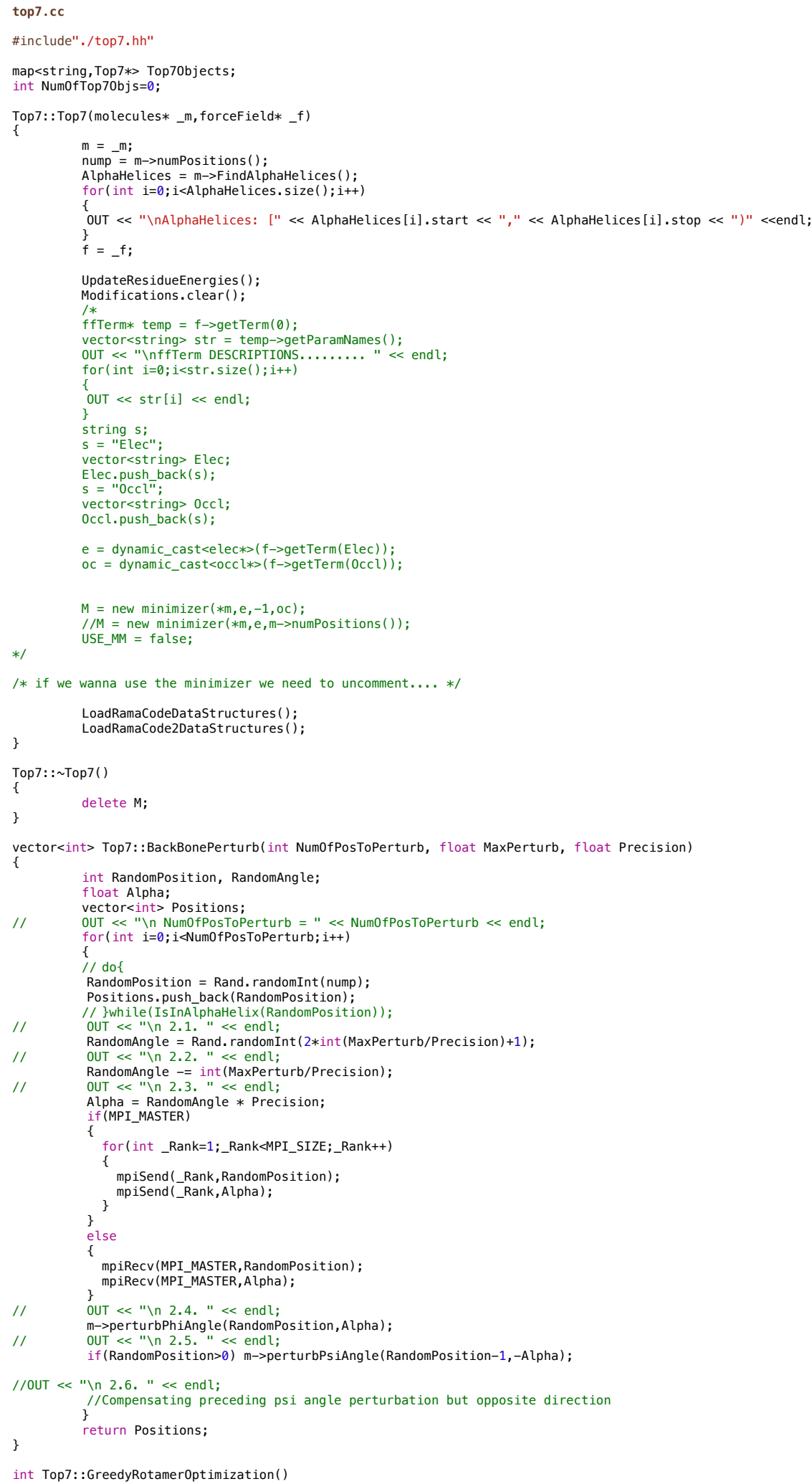

int Top7: : GreedyRotamer0ptimization() 


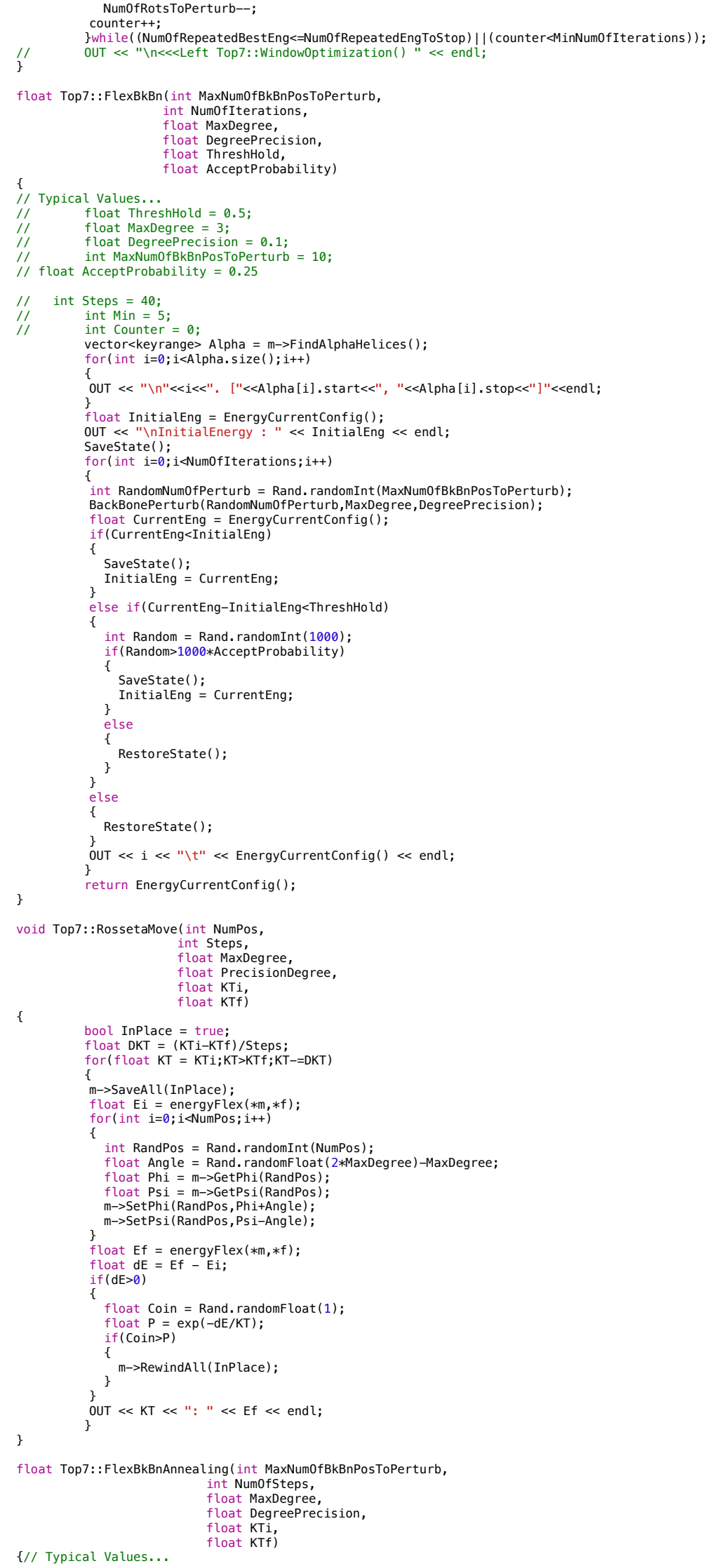




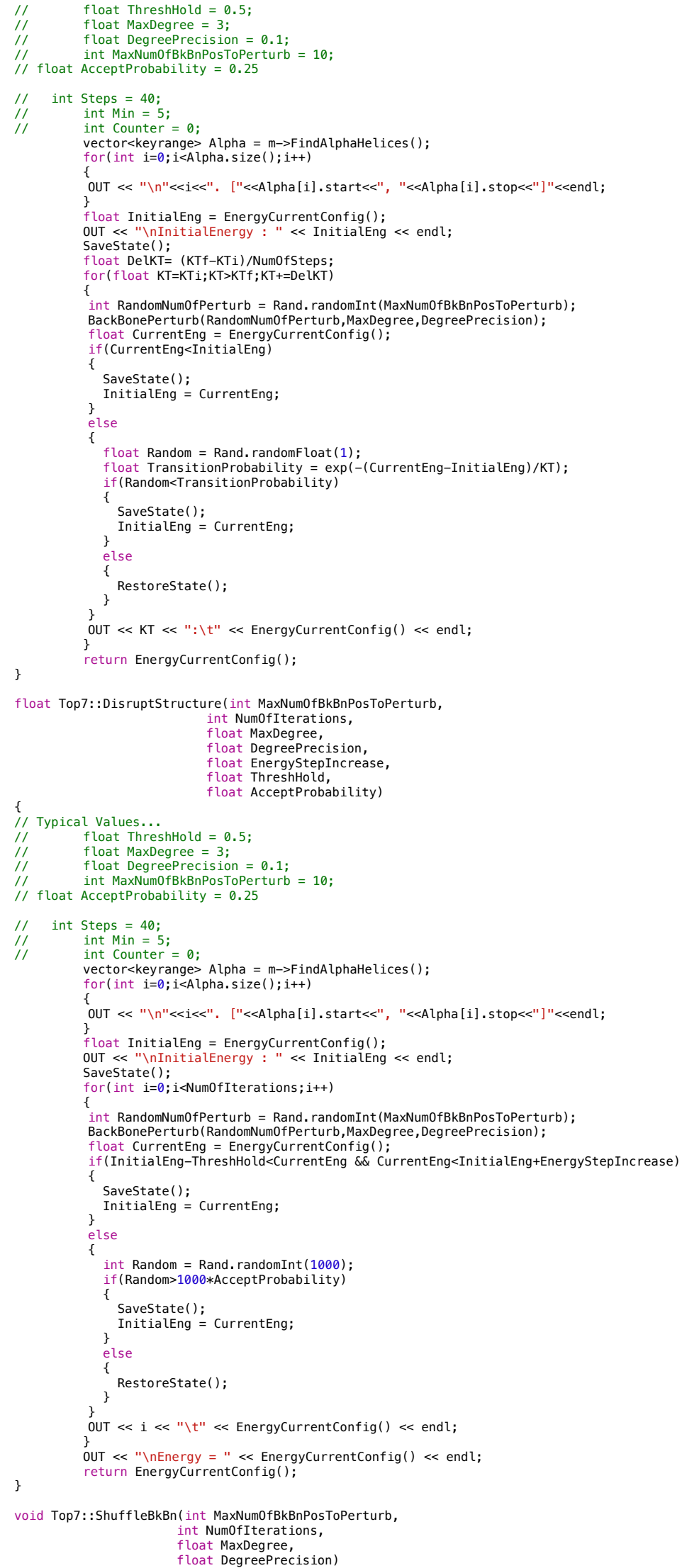




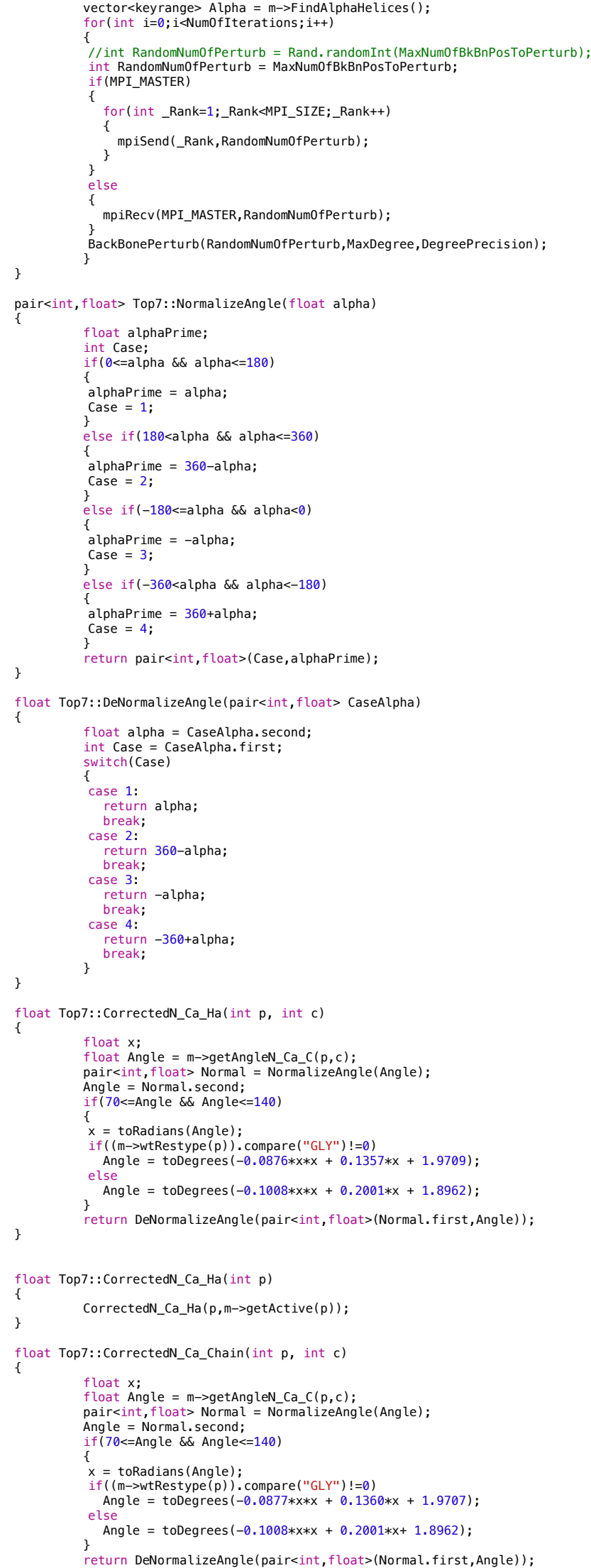


float Top7::CorrectedN_Ca_Chain(int p)

CorrectedN_Ca_Chain ( $p, m->\operatorname{get} A c t i v e(p))$;

float Top7: :CorrectedC_N_Ca_Ha(int $p$, int c)

float $x$;

float Angle $=m->$ getAngleN_Ca_C $(p, c)$;

pair $<$ int, float $>$ Normal $=$ NormalizeAngle $($ Angle $) ;$

Angle $=$ Normal. Second;
if $(70<=$ Angle $\& \&$ Angle $<=140)$

$x=$ toRadians (Angle);

(

Angle $=$ toDegrees $(-0.0719 * x * x+0.5080 * x+1.3853)$

Angle $=$ toDegrees $(-0.0790 * x * x+0.5629 * x+1.3072) ;$

\}

return DeNormalizeAngle(pair $<$ int, float $>$ (Normal. first, Angle));

float Top7: : CorrectedC_N_Ca_Ha (int p)

CorrectedC_N_Ca_Ha $(p, m->\operatorname{get} A c t i v e(p))$;

float Top7: : CorrectedC_N_Ca_Chain(int p, int c)

float $x$;

float Angle $=m \rightarrow$ getAnglen $C a+c(p, c)$;

pair<int, float $>$ Normal $=$ NormalizeAngle $($ Angle $) ;$

Angle $=$ Normal . second;

if $(70<=$ Angle \&\& Angle $<=140)$

\{

$x=$ toRadians (Angle);

f ( (m->WtRestype (p)). compare ("GLY") !=0

Angle $=$ toDegrees $(0.0719 * x * x-0.5081 * x-1.3853)$;

Angle $=$ toDegrees $(0.0790 * x * x-0.5629 * x-1.3072)$

\}

\}

return -DeNormalizeAngle(pair<int, float $>$ (Normal.first,Angle));

float Top7: :CorrectedC_N_Ca_Chain(int p)

CorrectedC_N_Ca_Chain $(p, m-\rightarrow \operatorname{get} \operatorname{Active}(p))$;

void Top7: : FixHa(int $p$, int $c$ )

I/ OUT $\ll$ "\n-_-_-_-_endl;

// OUT $\ll$ "\nAngleHa was: " $\ll m->$ getAngleHa $(\mathrm{p}, \mathrm{c}) \ll$ end

$/ / \quad$ OUT $\ll$ "InCorrectedHa_Angle: " $\ll$ CorrectedN_Ca_Ha(p, $\mathrm{c}) \ll$ endl;

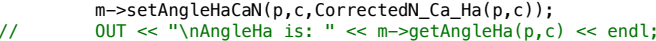

// OUT $\ll$ "\nDihedralHa was: " $\ll$ m->getDihedralHa $(p, c) \ll$ endl;

// OUT $\ll$ "InCorrectedDihedralHa_Angle: " $\ll$ CorrectedC_N_Ca_Ha(p,c) $\ll$ endl;

// OUT $\ll$ "InDihedralHa is: " 2 m->getDihedraitHa(p, c) $\ll$ endl;

void Top7: :FixHa(int p)

FixHa $(p, m->\operatorname{getActive}(p))$;

void Top7: : FixChain(int $p$, int $c$ )

\{/ OUT $\ll$ "InAlpha: " $<$ m->getAngleN_Ca_C $(p, c) \ll$ endl;

OUT $\ll$ "InAngleChain was: " $\ll$ m $\rightarrow$ getAngleChain $(\mathrm{p}, \mathrm{c}) \ll$ endl;

// OUT $\ll$ "InCorrectedChain_Angle: " $\ll$ CorrectedN_Ca_Chain $(p, c) \ll$ endl;

$m \rightarrow \operatorname{set}$ AngleChainCaN $(p, c$, CorrectedN_Ca_Chain $(p, c))$;

OUT $\ll$ "\nAnglechain is: " $\ll$ m- getAngleChain $(\mathrm{p}, \mathrm{c}) \ll$ endl

// OUT $\ll$ "InDihedralChain was: " $\ll$ m- $>$ getDihedralChain $(p, c) \ll$ end

// OUT $\ll$ "\nCorrectedDihedralChain_Angle: " $\ll$ CorrectedC_N_Ca_Chain $(p, c) \ll$ endl; OUT $\ll$ "\nDihedratchain is: " $\ll$ m->getDihedratchain $(\mathrm{p}, \mathrm{c}) \ll$ endl;

void Top7: : FixChain (int $\mathrm{p}$ )

FixChain $(p, m->\operatorname{getActive}(p))$;

void Top7: :BackrubMove(int p1, int $\mathrm{p} 2$, float theta, int FixAtoms)

if $(\mathrm{p} 1<0 \quad|| \mathrm{p} 1>=\mathrm{m}->$ numPositions ()$)$

throw Top7Error_BadPosition("BackrubMove", p1)

else if $(\mathrm{p} 2<0 \quad|| \mathrm{p} 2>=\mathrm{m}->$ numPositions ())

if $((m->$ wtRestype( 1$))$. compare ("GLY")==0|| ( $(m->$ wtRestype $(p 2))$. compare ("GLY" $)==0)$

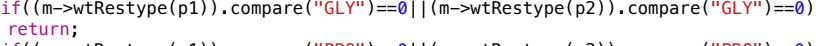

if $((\mathrm{m} \rightarrow \mathrm{w}, \mathrm{R})$

return;

const keyrange\& key $1=m->\operatorname{get} \operatorname{Conf}(\mathrm{p} 1)$;

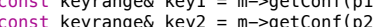

int $\mathrm{Ca} 1=$ getAtom $(\mathrm{m} \rightarrow>$ atoms, $\mathrm{key} 1$, padstringCA);

int $\mathrm{Ca} 2=$ getAtom $(\mathrm{m}->$ atoms, key2, padstringCA $)$;

int $\mathrm{H1}=\operatorname{getAtom}(\mathrm{m}->$ atoms, key1, padstring $\mathrm{H})$ :

int $\mathrm{H} 2=$ getAtom ( $\mathrm{m}->$ atoms, $\mathrm{key} 2$, padstringH) 


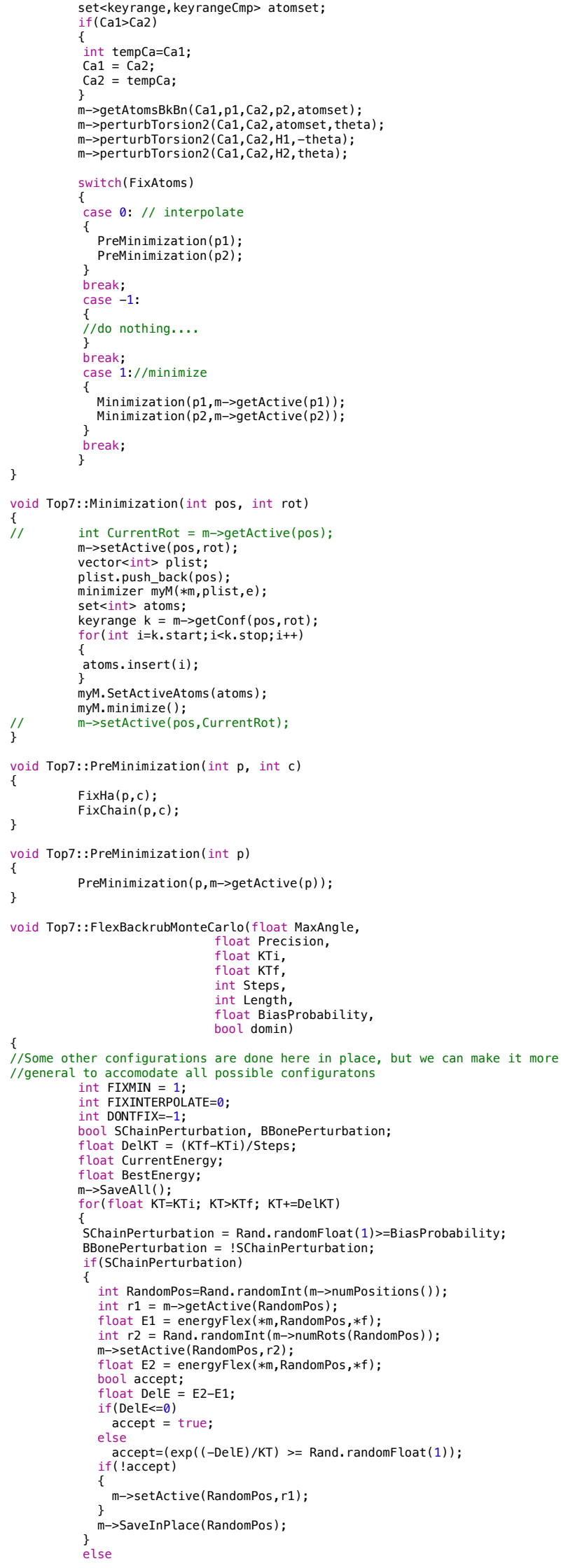


int HeadPos $=$ Rand , randomInt ( $m->$ numPositions ()$-$ Length) ;

int TailPos $=$ HeadPos+Length:

$m \rightarrow$ SaveInPlace (HeadPos);

$\mathrm{m} \rightarrow>$ SaveInPlace (TailPos):

int NumOfGrids = ceil(MaxAngle/Precision);

float Angle $=$ Precision $*$ (Rand . randomInt $(2 *$ NumOfGrids $)$-Num0fGrids);

float $\mathrm{E1}=\operatorname{energyFlex}(* \mathrm{~m}, * \mathrm{f}) ; / / /$ energyFlexWindow $(* m$, HeadPos, TailPos $+1, * \mathrm{f})$;

if (domin)

BackrubMove(HeadPos, TailPos, Ang le, FIXMIN);

BackrubMove (HeadPos, TailPos, Ang le, FIXINTERPOLATE)

float $\mathrm{E2}=\operatorname{energyFlex}(* \mathrm{~m}, * \mathrm{f}) ; / / /$ energyFlexWindow $(* \mathrm{~m}$, HeadPos,TailPos $+1, * \mathrm{f})$;

float DelE = E2-E1;

bool accept;

accept = true;

accept
else

accept $=(\exp (-\operatorname{DelE} / \mathrm{KT})>=$ Rand. $\operatorname{randomFloat}(1))$,

if ( !accept)

$/ / m \rightarrow$ Rewindall ( )

BackrubMove (HeadPos, TailPos,-Angle, DONTFIX);

$m \rightarrow>$ RewindInPlace (HeadPos);

else

vector<int> Positions;

for ( int Pos=HeadPos; Pos<=TailPos; Pos++)

Positions.push_back(Pos);

$m \rightarrow$ ReAlignRotamers (Positions):

for (int rot $=1$; rot $<m->$ numRots (HeadPos); rot++)

\{

if(!domin)

PreMinimization (HeadPos, rot)

Minimization (HeadPos, rot);

for (int $\operatorname{rot}=1$; $\operatorname{rot}<m->$ numRots (TailPos); rot ++ )

$\{$ if (!domin)

if(!domin)

else

\}

Minimization(TailPos, rot);

\}

$\rightarrow$ SaveAll(true);

CurrentEnergy $=$ energyFlex $(* \mathrm{~m}, * \mathrm{f})$;

OUT $\ll$ KT $\ll$ "\t: " $<$ CurrentEnergy $\ll$ endl;

if (CurrentEnergy<BestEnergy)

\{

\}

DestEnergy= CurrentEnergy

OUT $\ll$ "\n\nFlexBackrub: " $\ll$ BestEnergy $\ll$ endl;

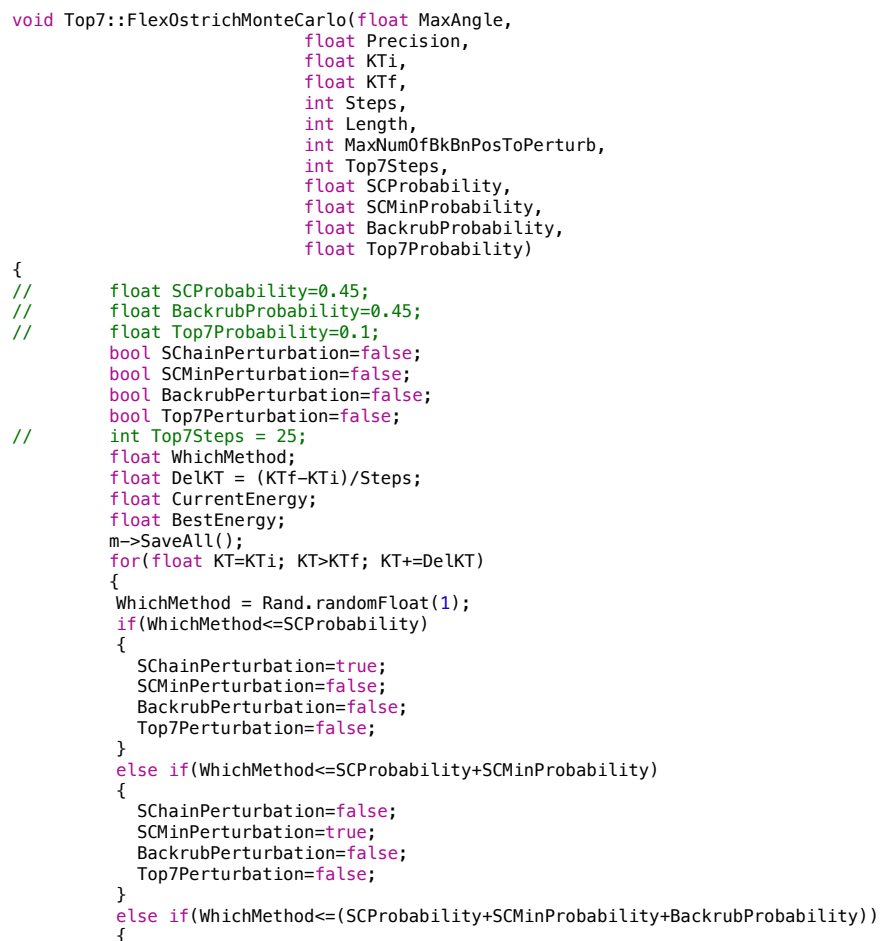




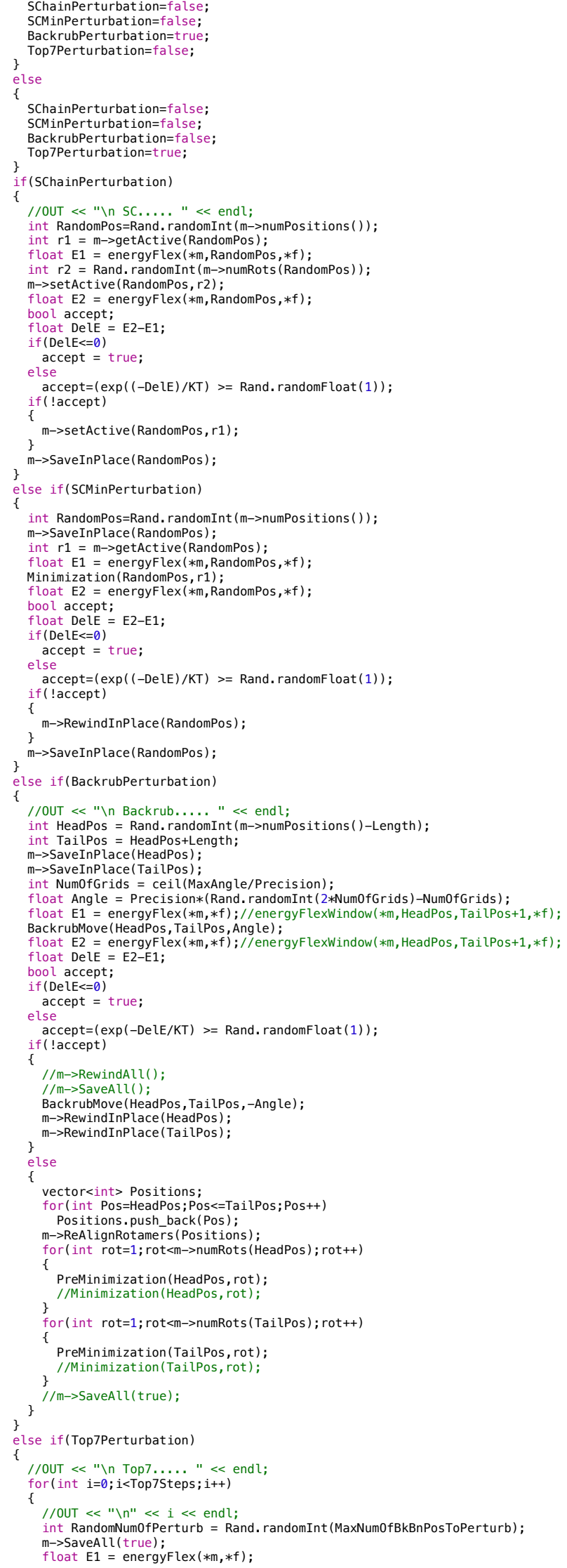




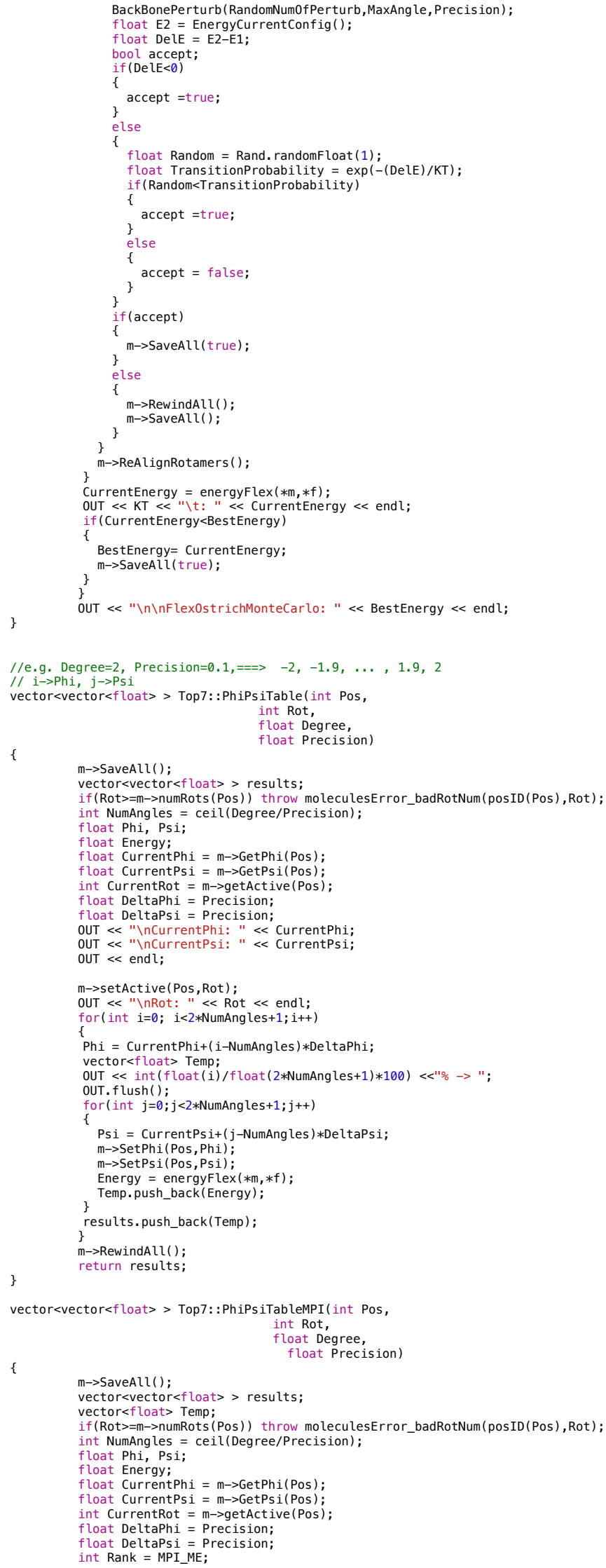




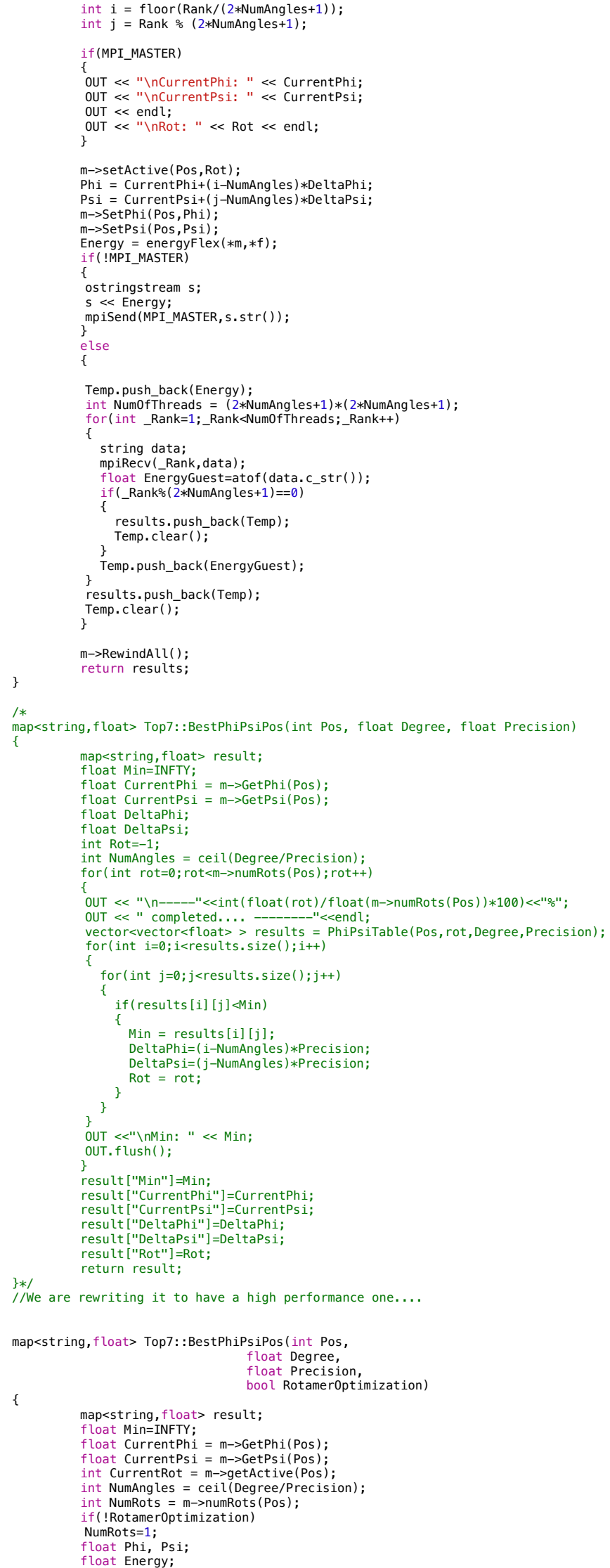




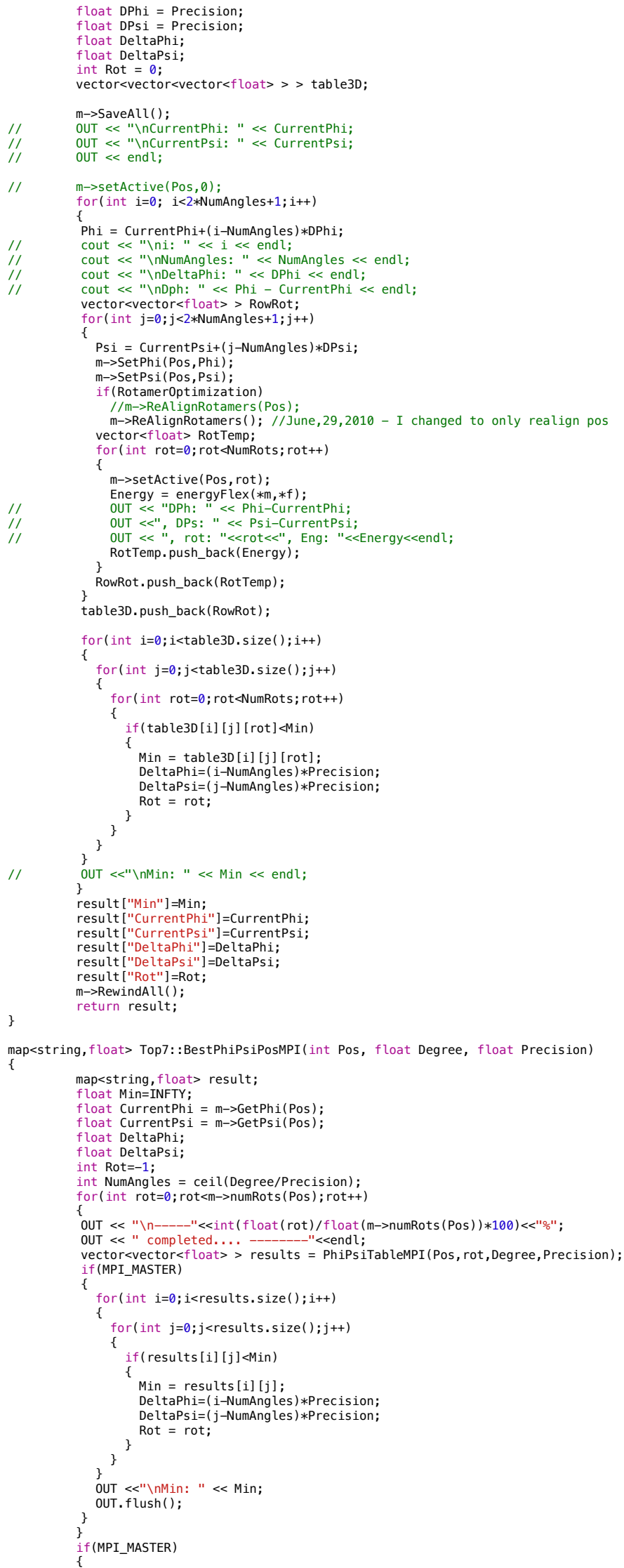




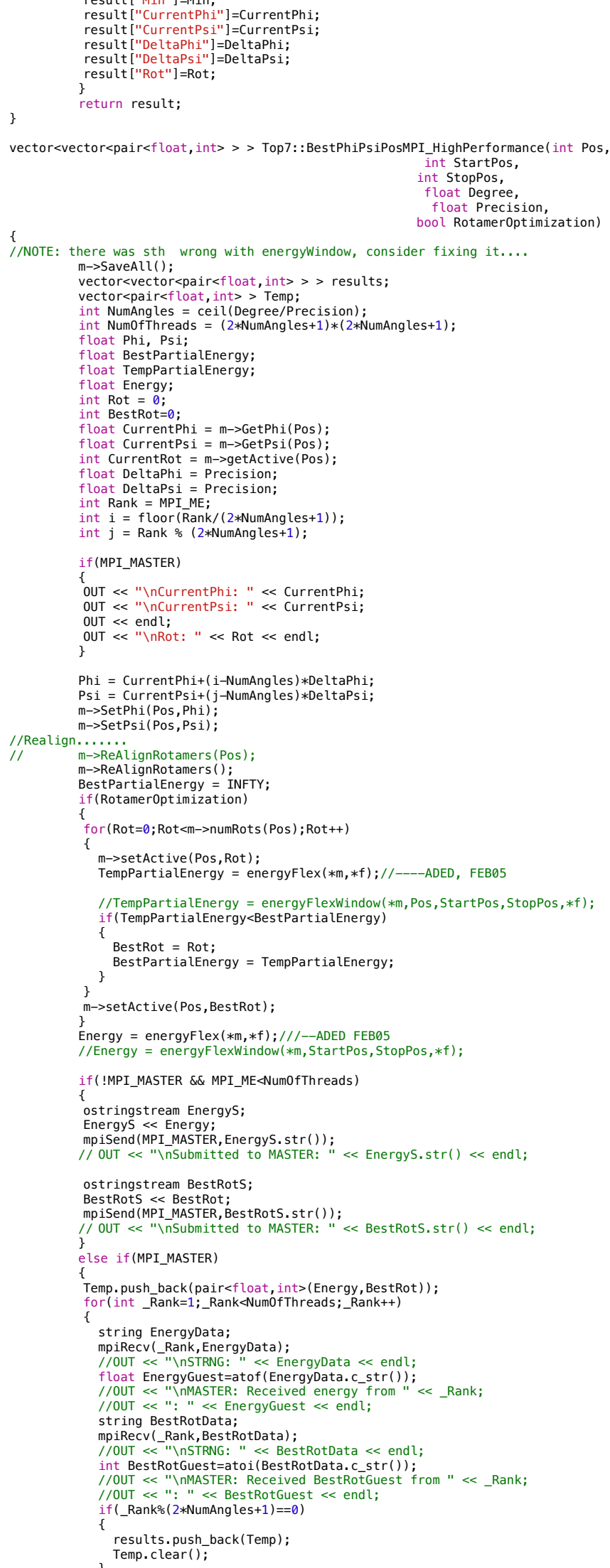




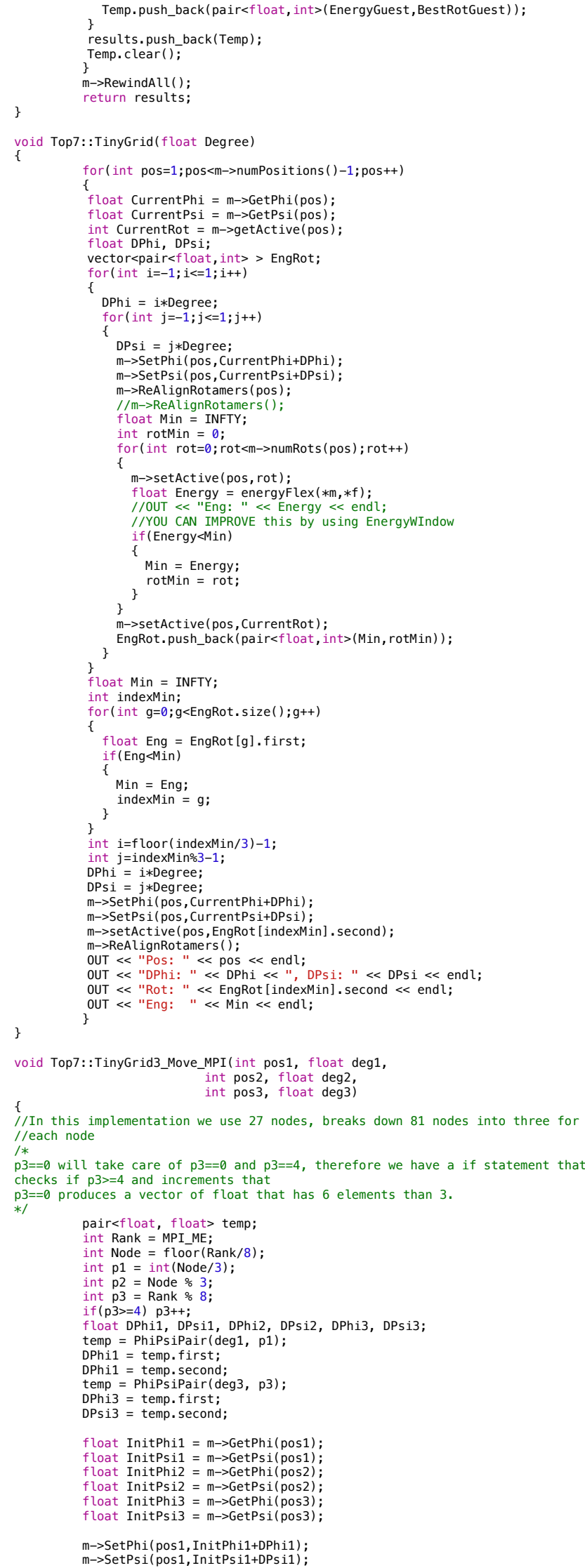




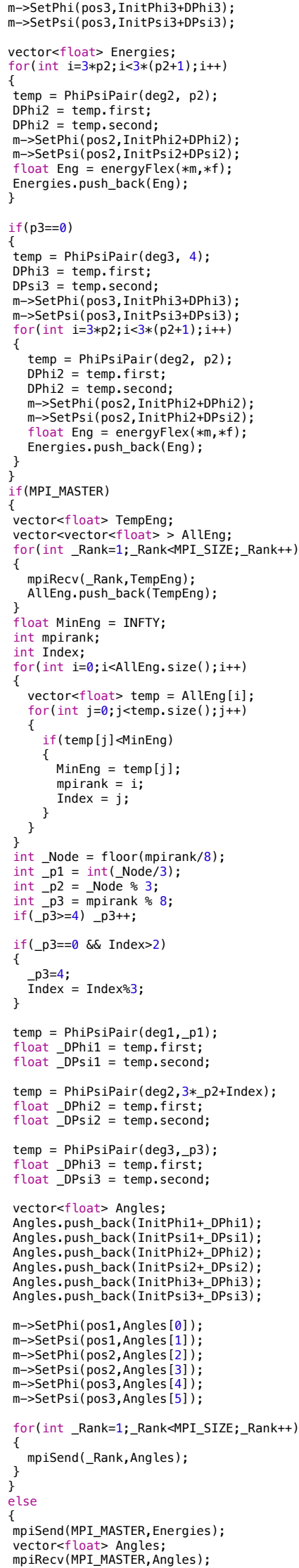




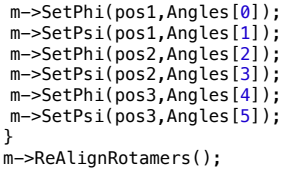

pair<float, float> Top7: :PhiPsiPair(float deg, int offset)

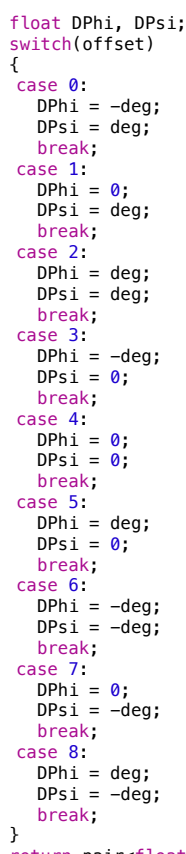




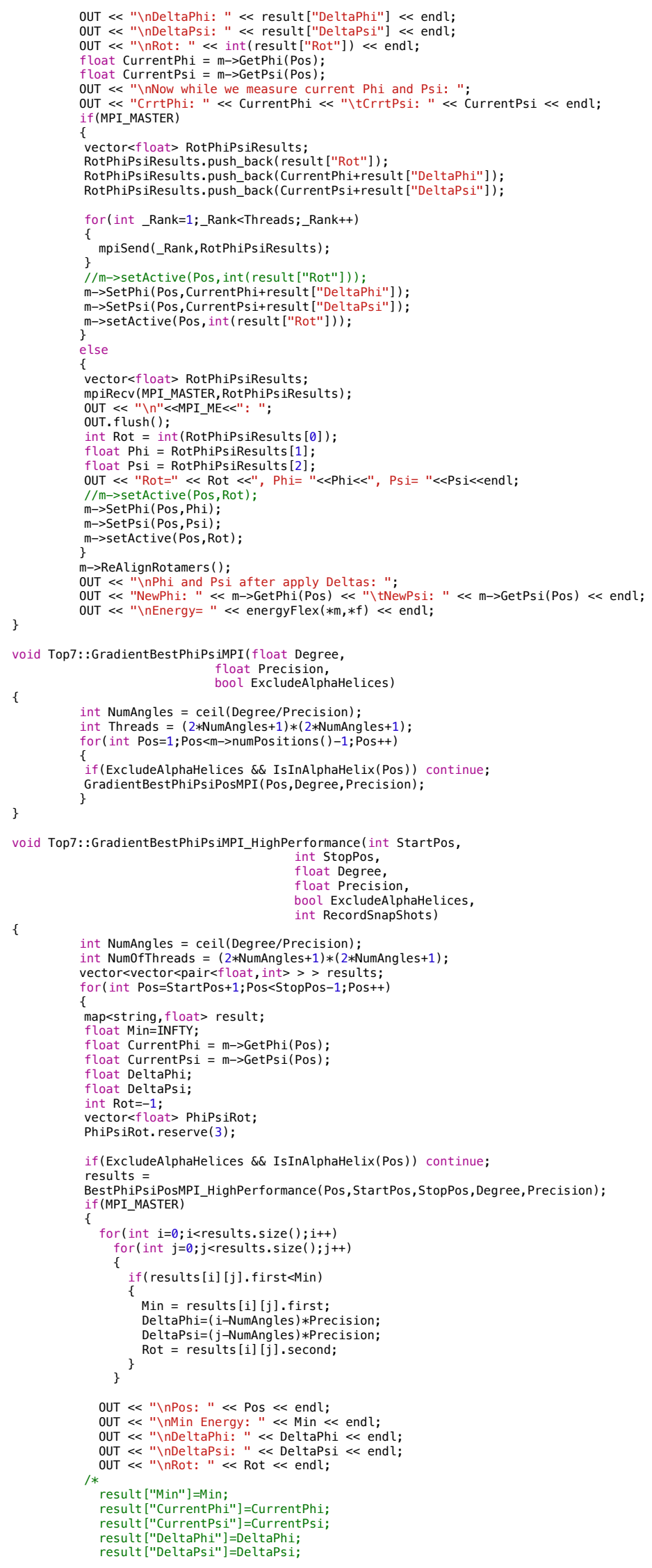




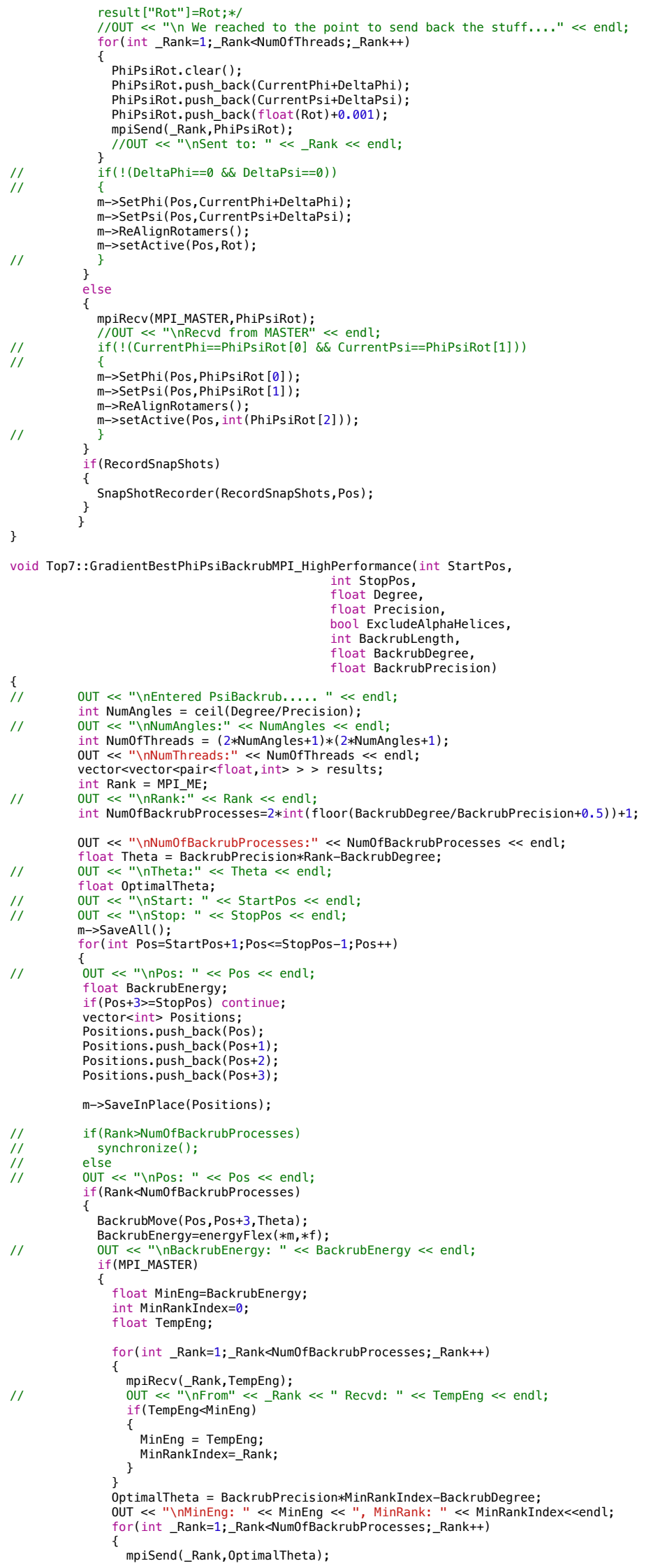




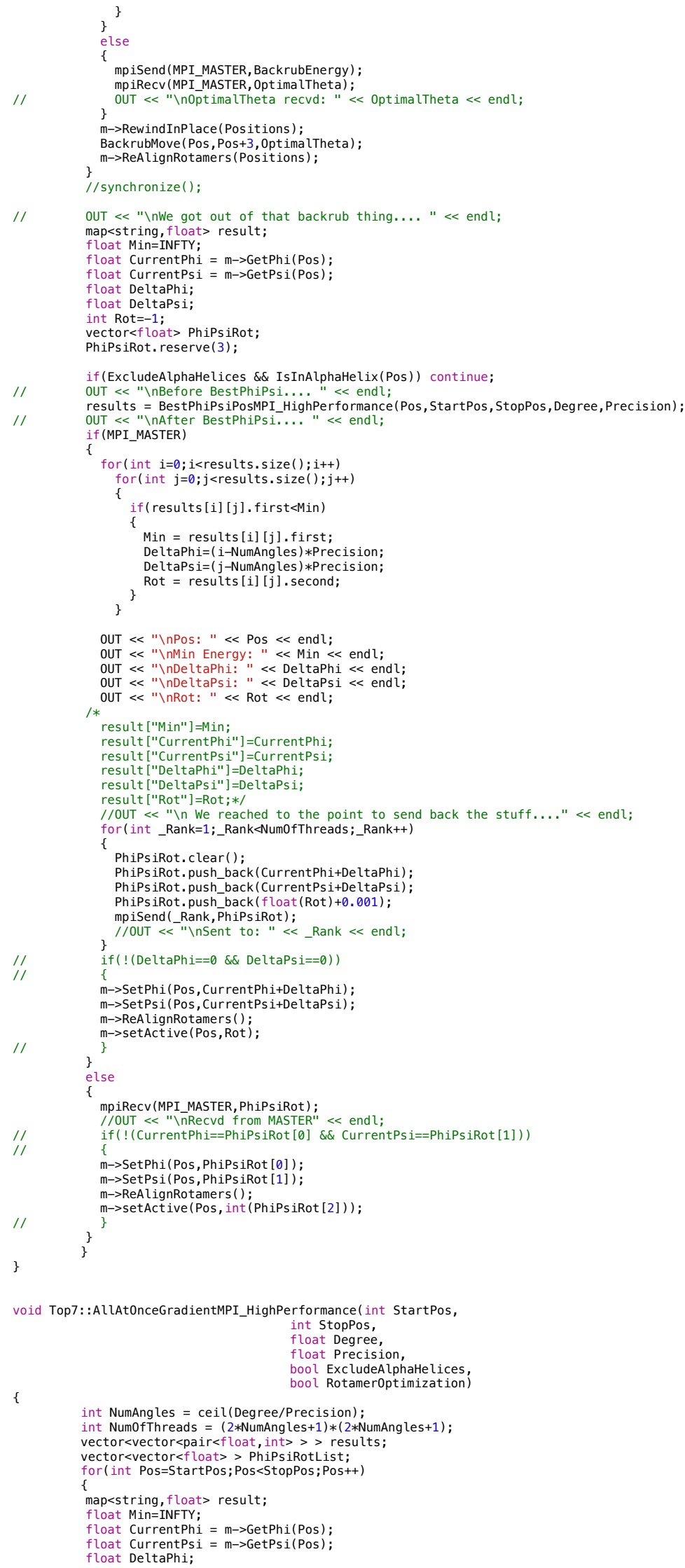




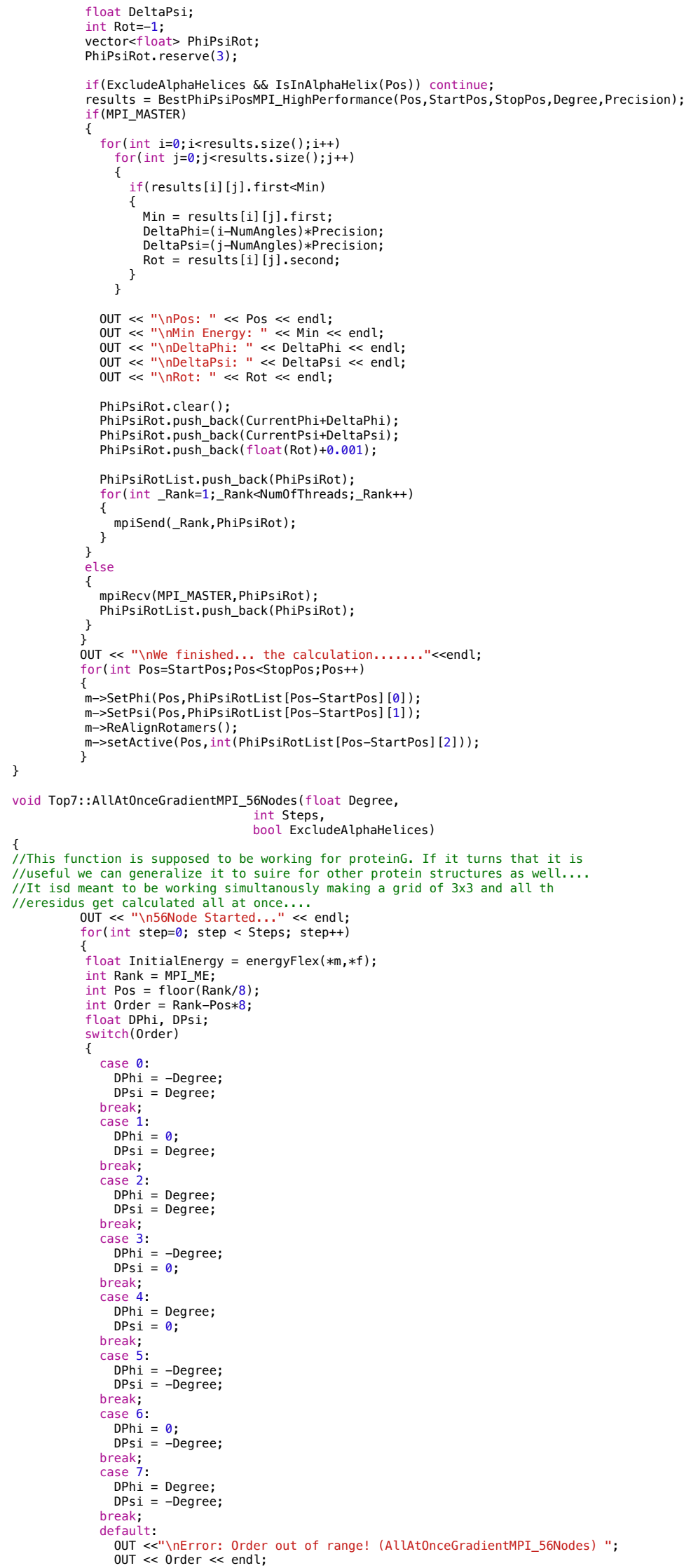




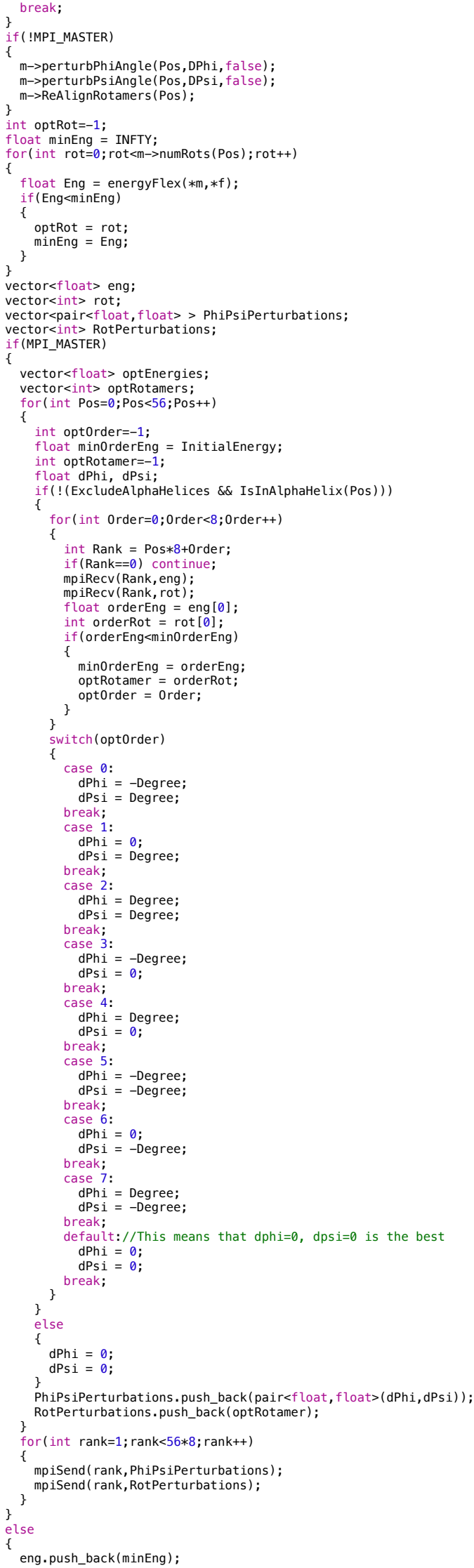

PhiPsiPerturbations. push_back (pair $<$ float, float $>(d P h i, d P s i)$ ) RotPerturbations,push back (optRotamer) 


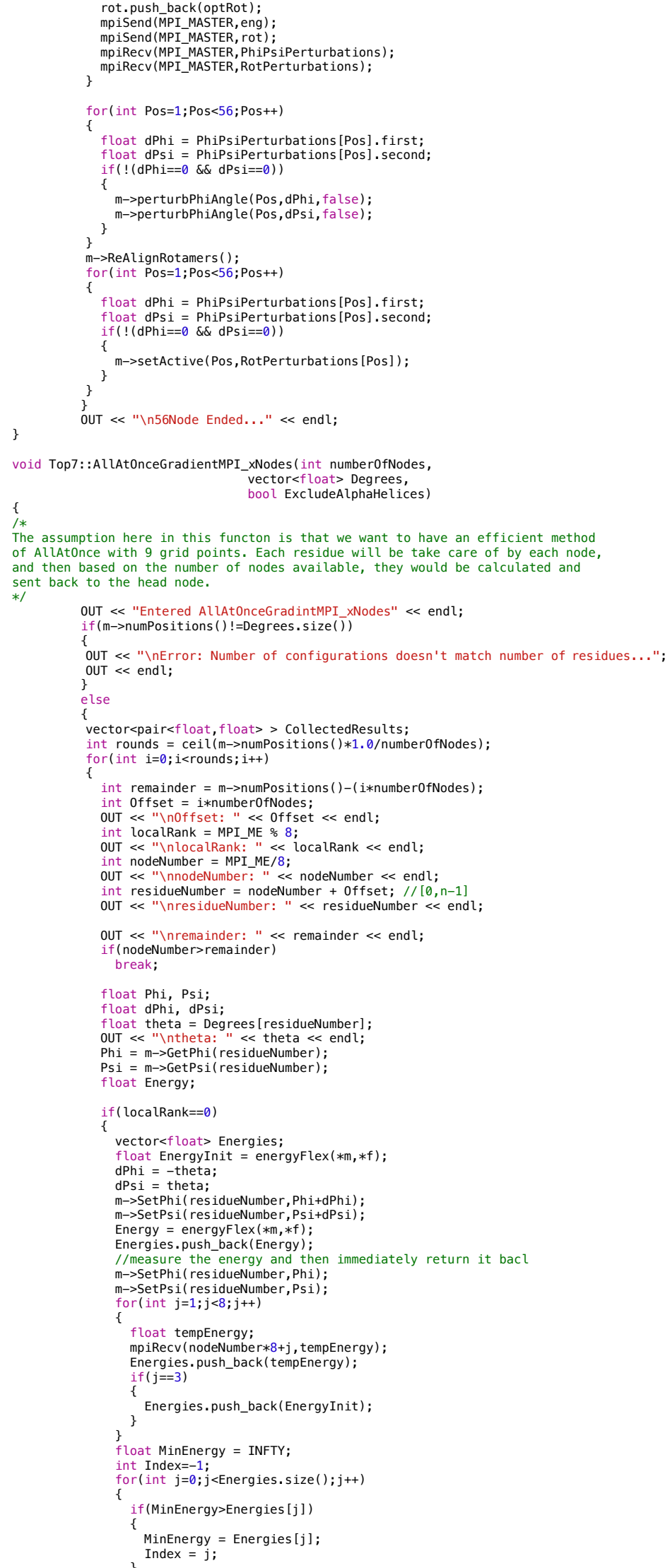




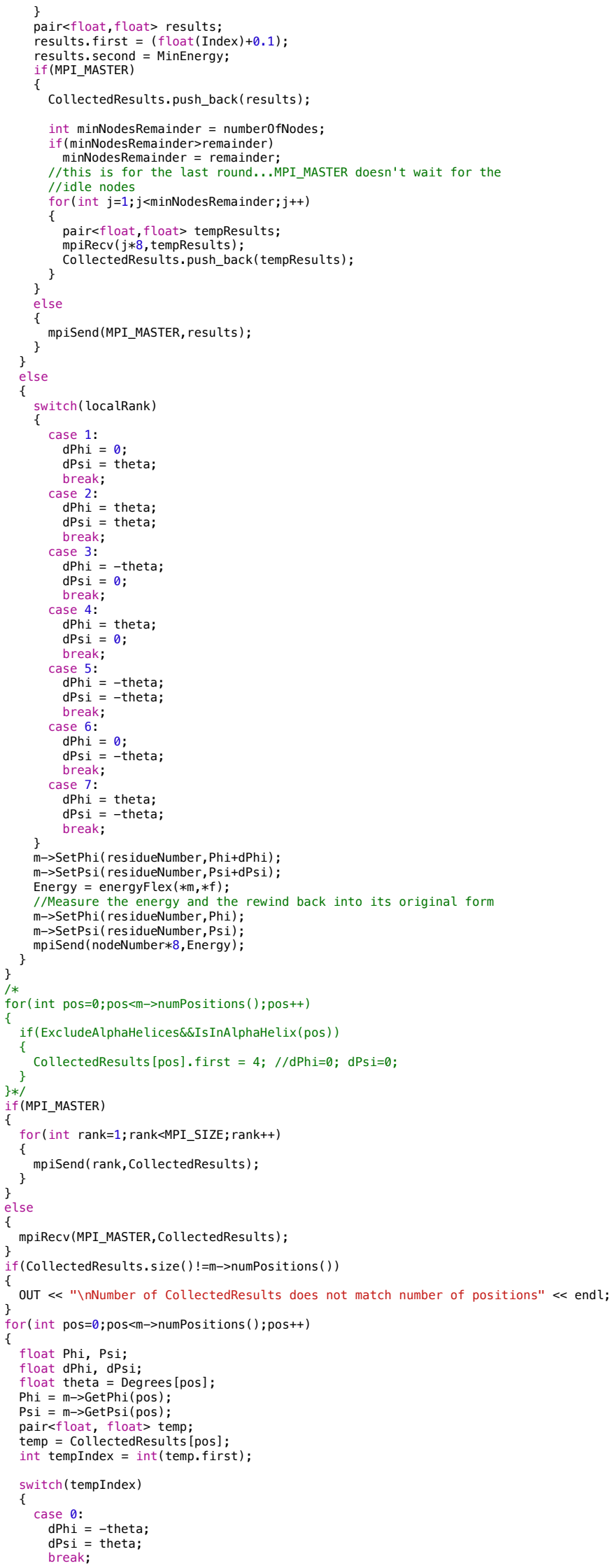




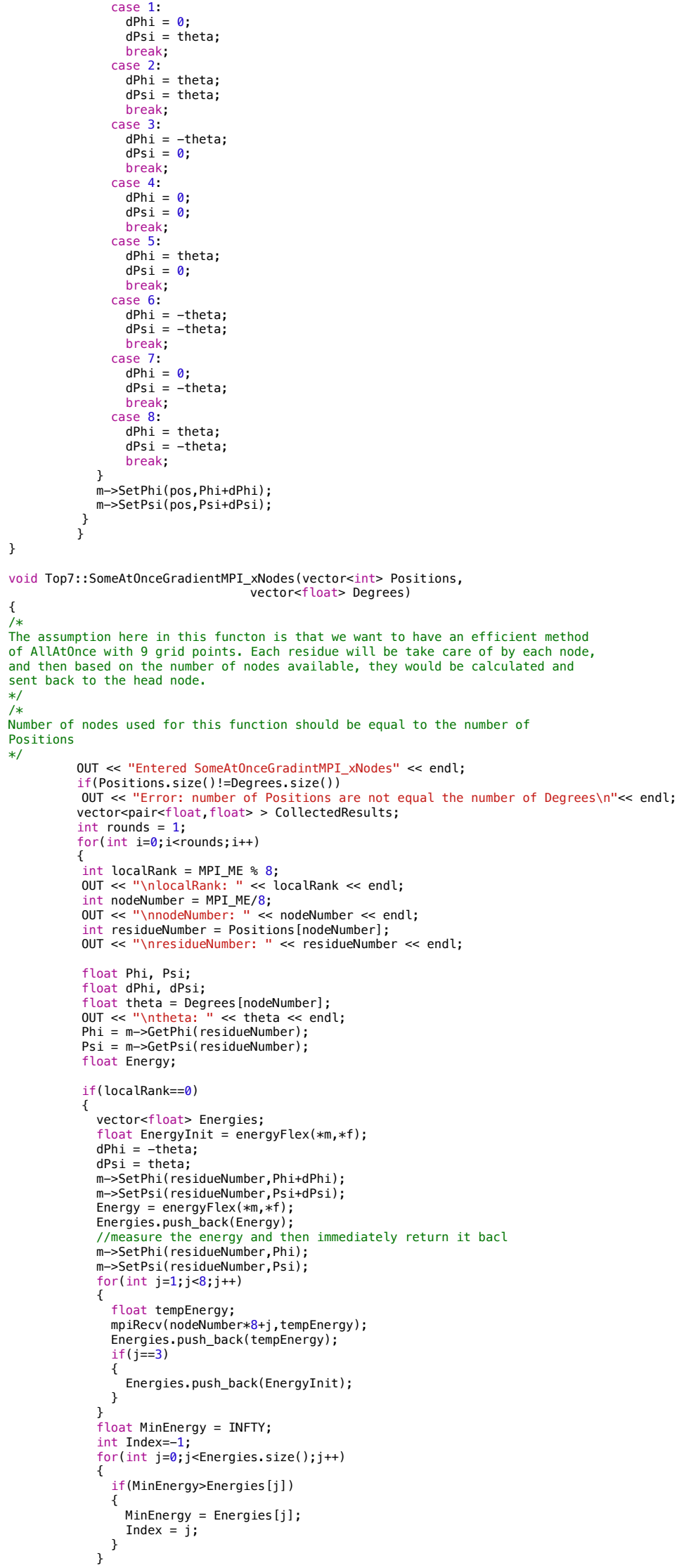




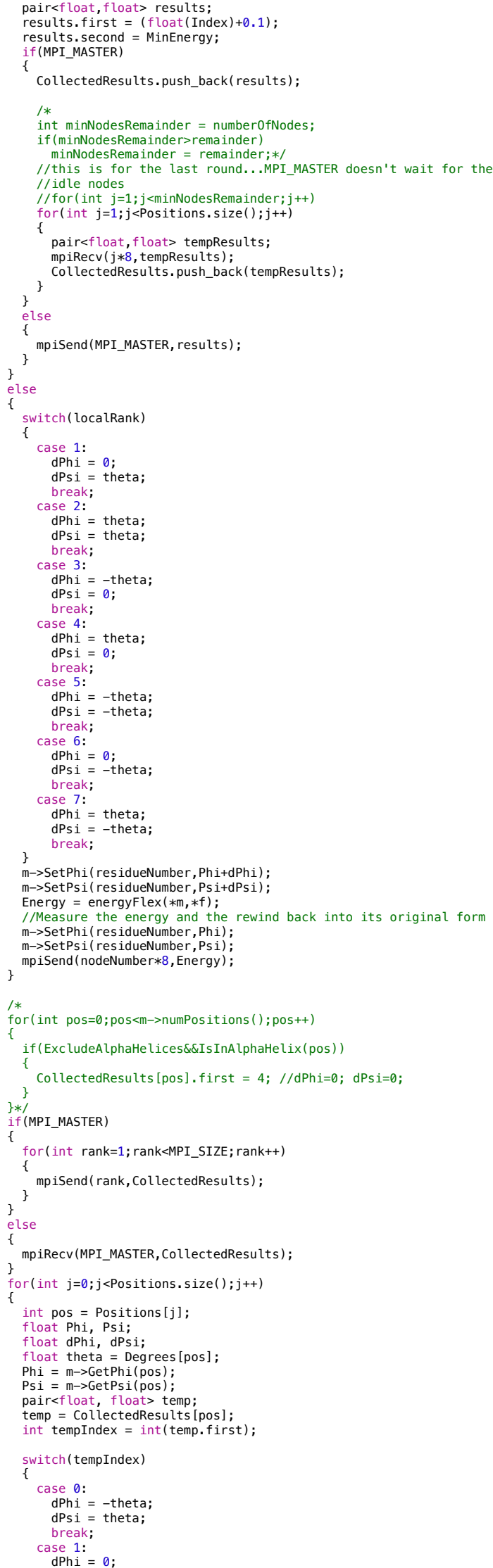




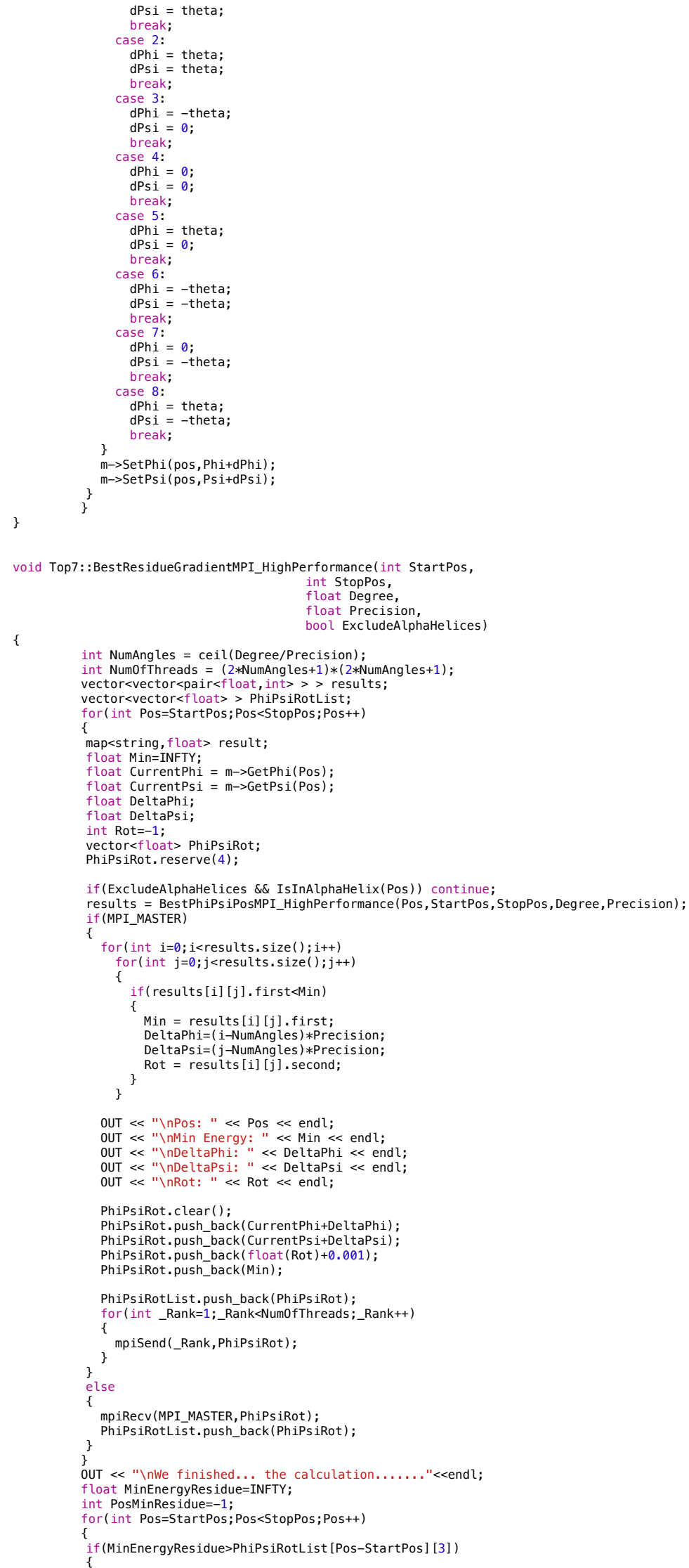




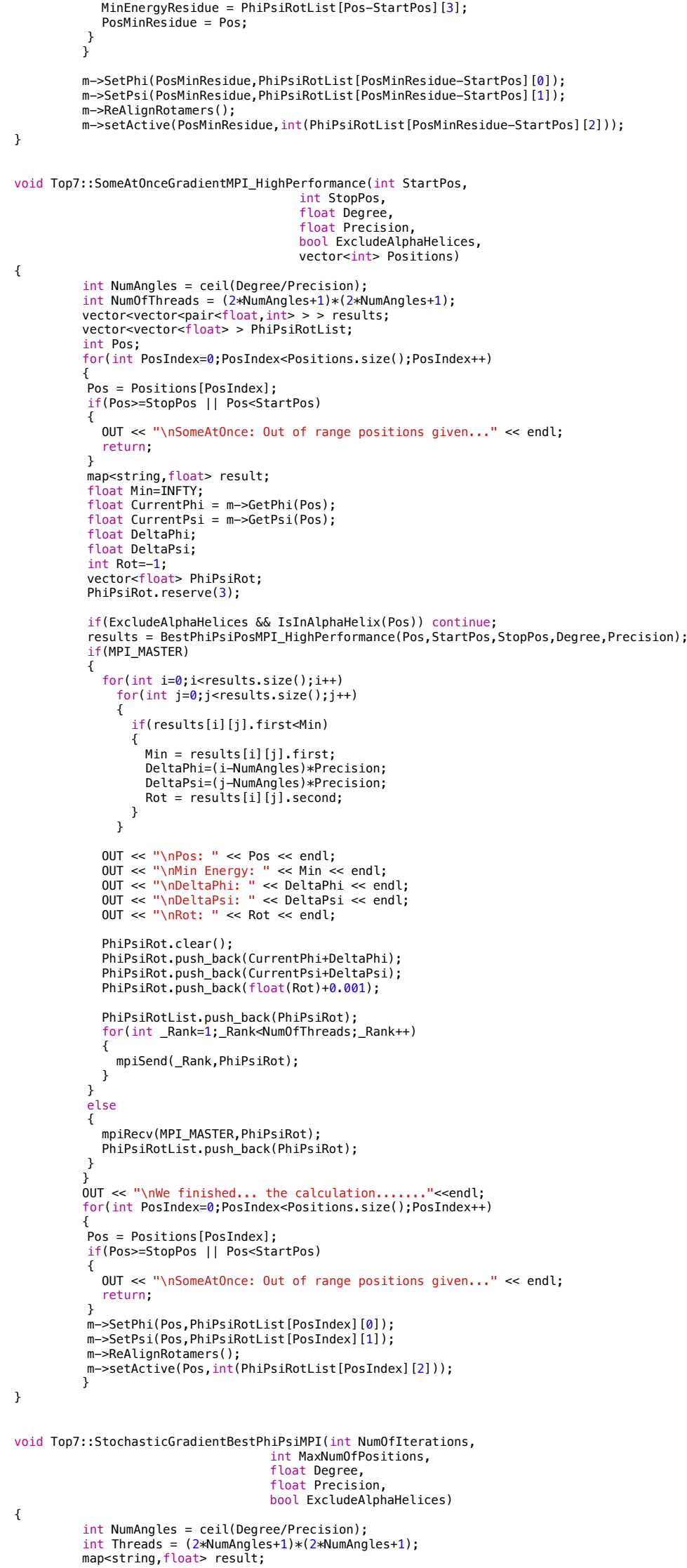




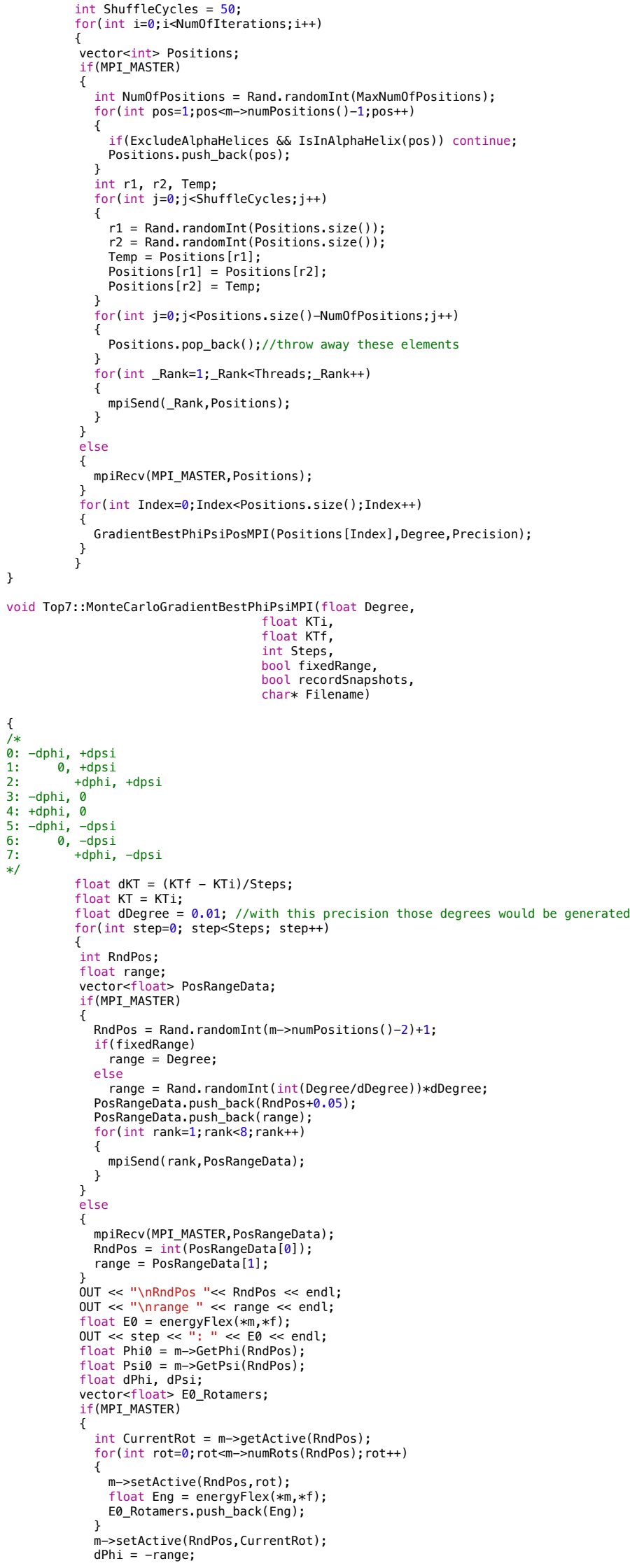




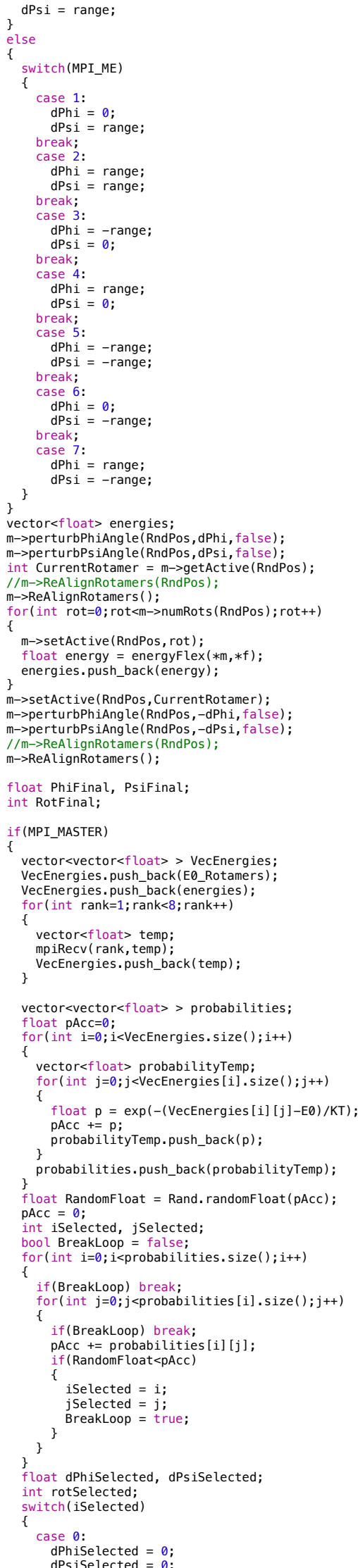




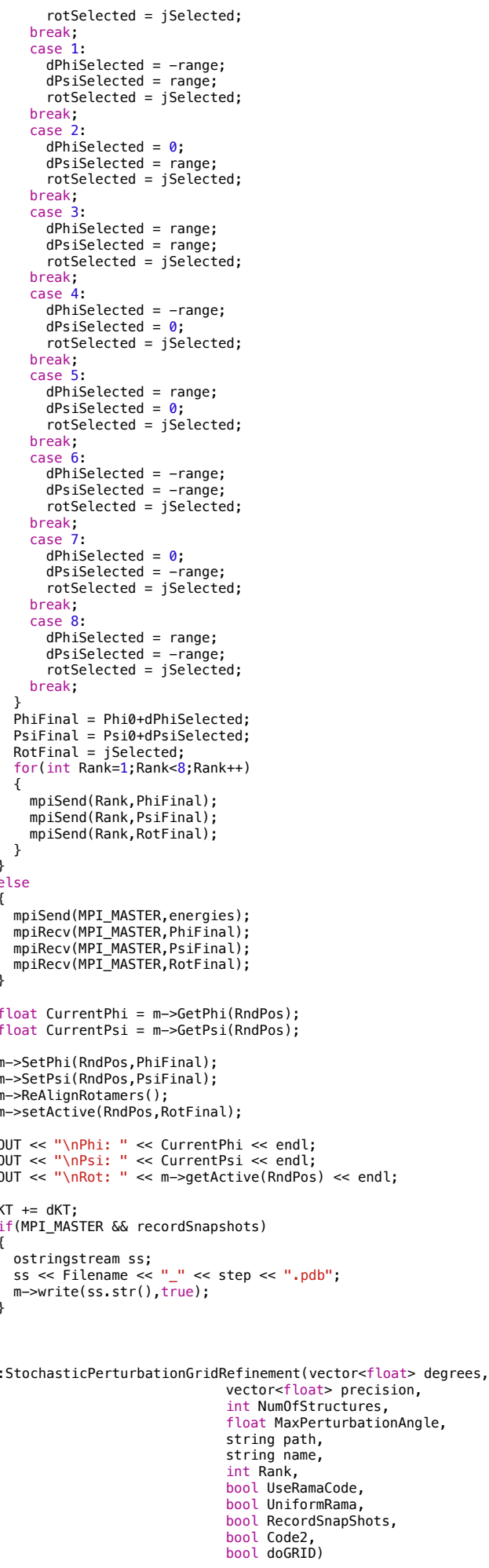

float E;

ostringstream StreamEnergyefore

StreamEnergyBefore $\ll$ path $\ll " / " \ll$ name $\ll " ~ " \ll$ Rank $\ll$ "Before.txt"; ofstream FileEnergyBefore (StreamEnergyBefore. $\operatorname{sir}($ ).c_str( )).

ostringstream StreamEnergyAfter;

« name $\ll "$ " $\ll$ Rank $\ll "$ ". txt",

ofstream FileEnergyAfter(StreamEnergyAfter.st $\bar{r}()$. c_str());

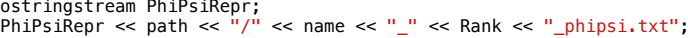


ofstream FilePhiPsiRepr(PhiPsiRepr.str().c_str());

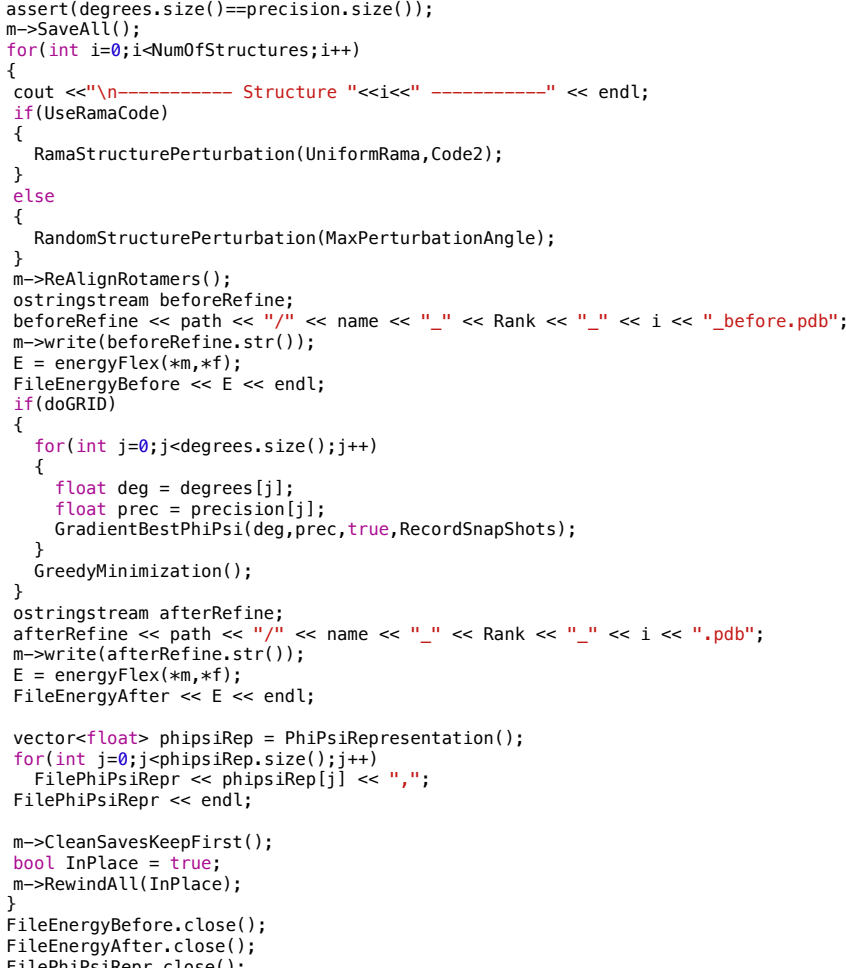

\{

OUT $\ll$ "We entered this module..." $\ll$ endl;

float $E_{\text {; }}$

ostringstream StreamEnergyBefore;

年 ofstream FileEnergyBefore (StreamEnergyBefore.st $\operatorname{tr}($ ).c_str());

ostringstream StreamEnergyAfter;

ofstream FileEnergyAfter(StreamEnergyAfter.st $\bar{r}()$. c_str()):

OUT $\ll$ "ready before saveall...." $\ll$ endl;

$m \rightarrow$ SaveAll():

$m->$ SaveAll( );
for (int $i=0 ; i<$ Num0fStructures; $i++)$

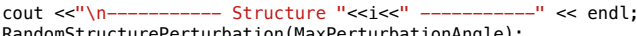

RandomStructurePerturbation (Mar

OUT $\ll<$ "Random Structure

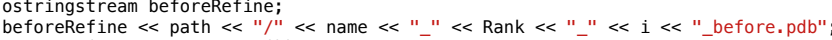

m->write(beforeRefine.str()):

$\mathrm{E}=\operatorname{energyFlex}(* \mathrm{~m}, * \mathrm{f})$

FileEnergyBefore $\ll \mathrm{E} \ll$ endl;

FlexBackrubMonteCarlo (MaxAngle, Precision, KTi, KTf, Steps, Length, BiasProbability, domin)

ostringstream afterRefineBeforeMin

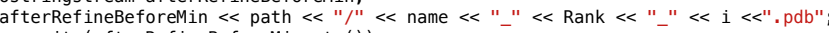

m->write (afterRefineBeforeMin.str( ))

GreedyMinimization( )

ostringstream afterRefine;

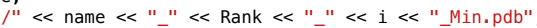

Exrite (afterRefine.str())

FileEnergyAfter $\ll<\mathrm{E} \ll<$ endl;

$\mathrm{m} \rightarrow$ CleanSavesKeepFirst ()

bool InPlace = true;

$m \rightarrow$ Rewindall (InPlace);

FileEnergyBefore.close(),

FileEnergyafter.close(): 


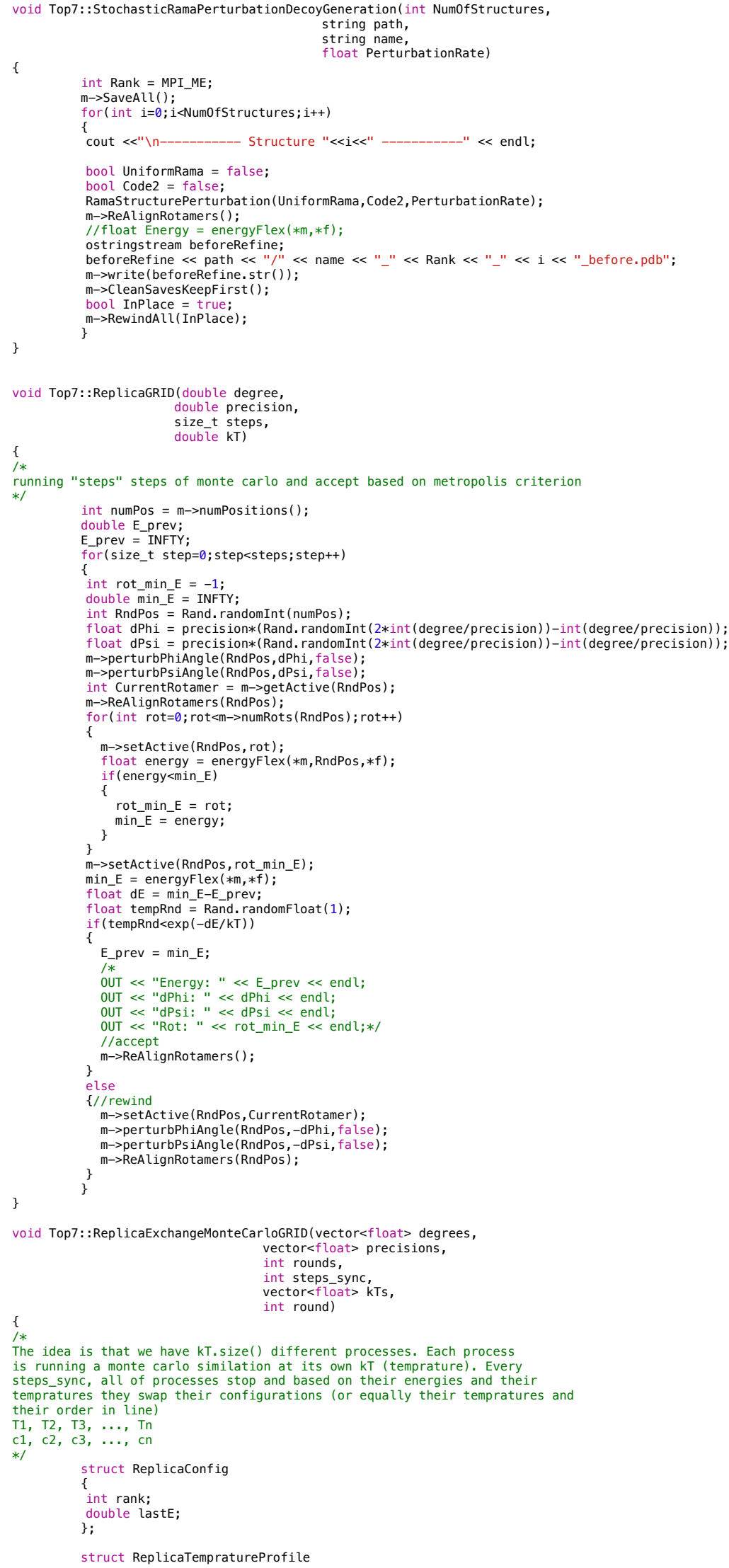




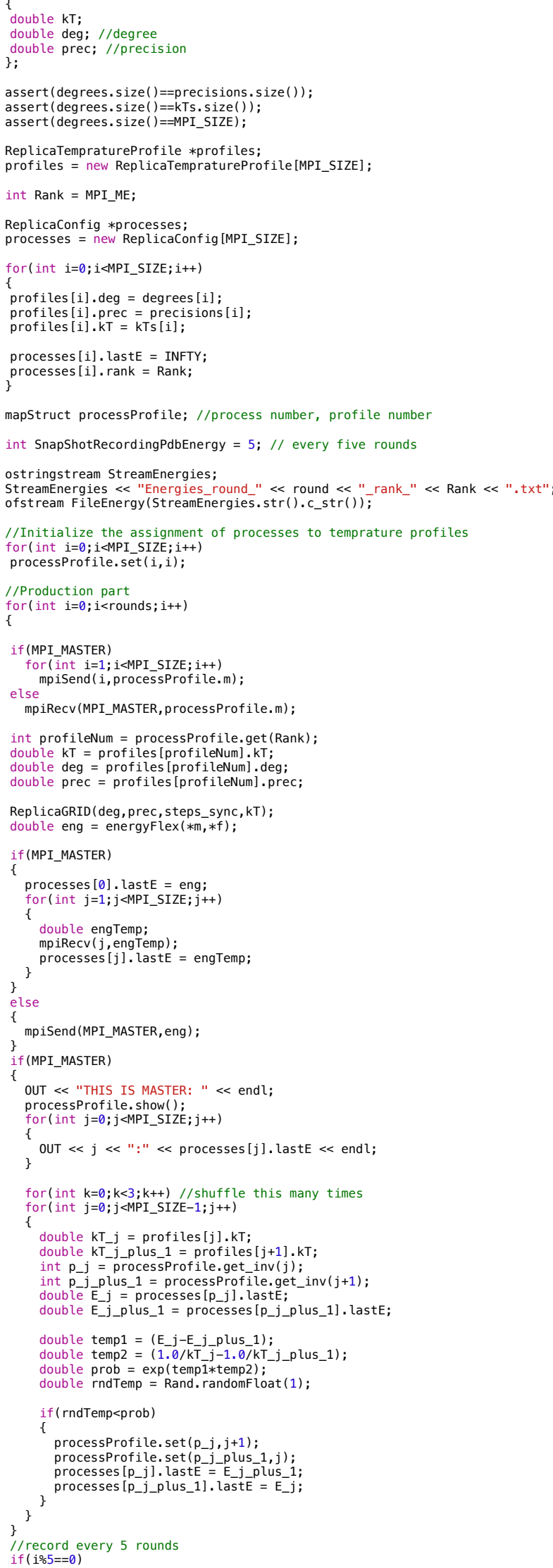




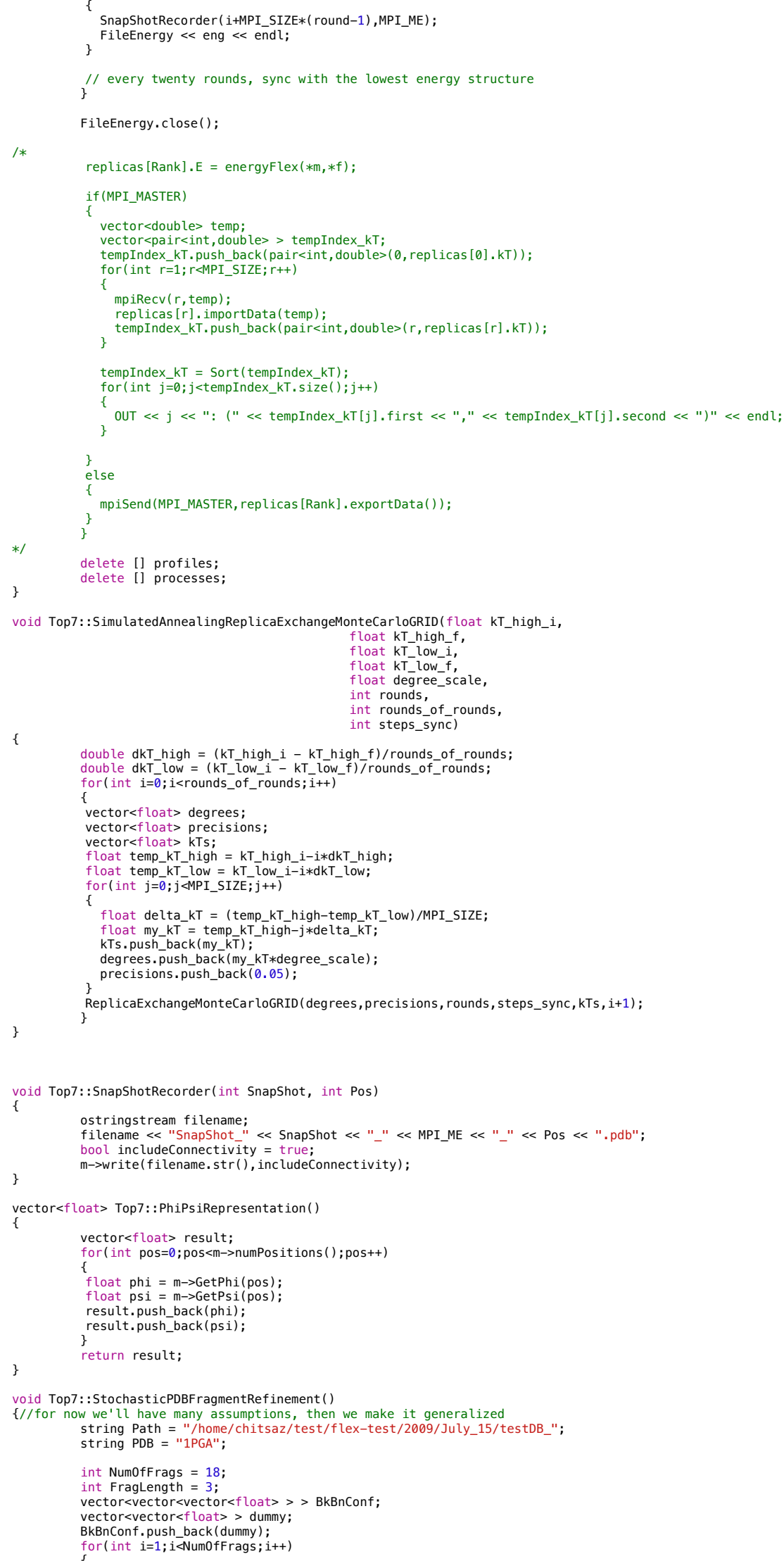




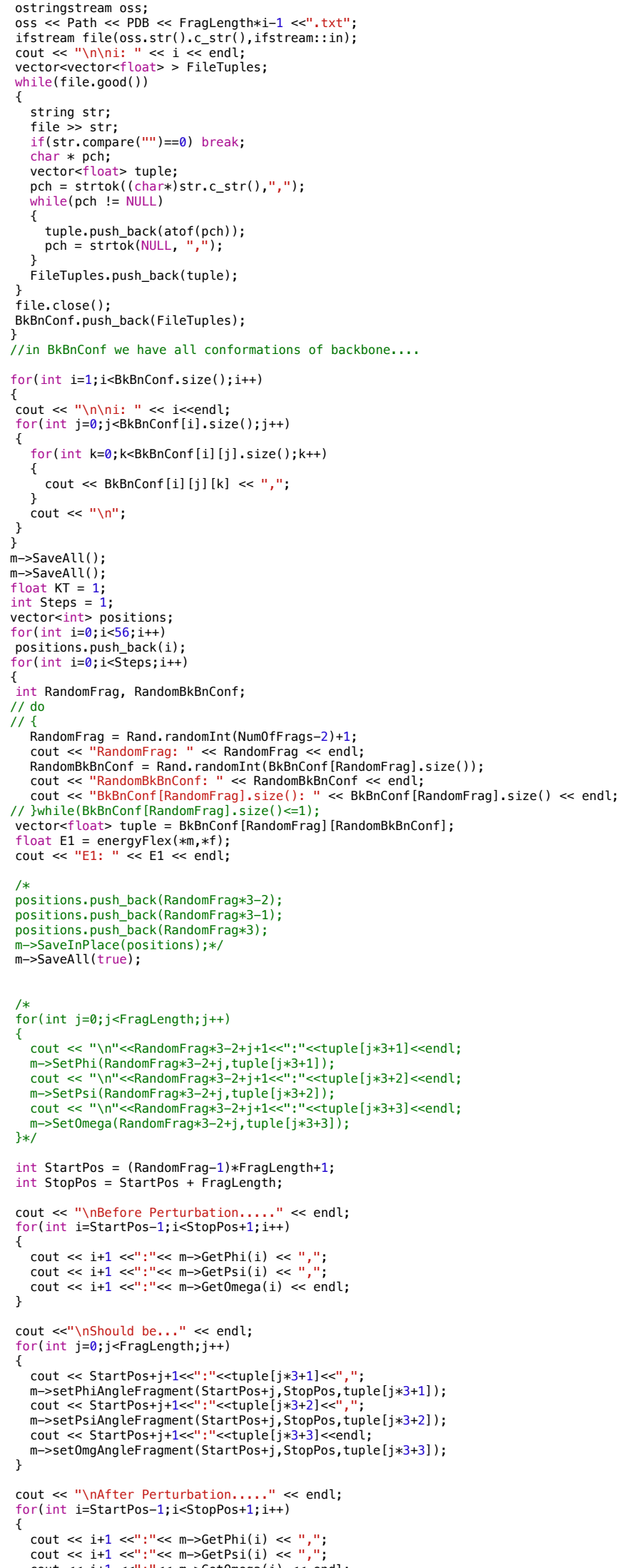




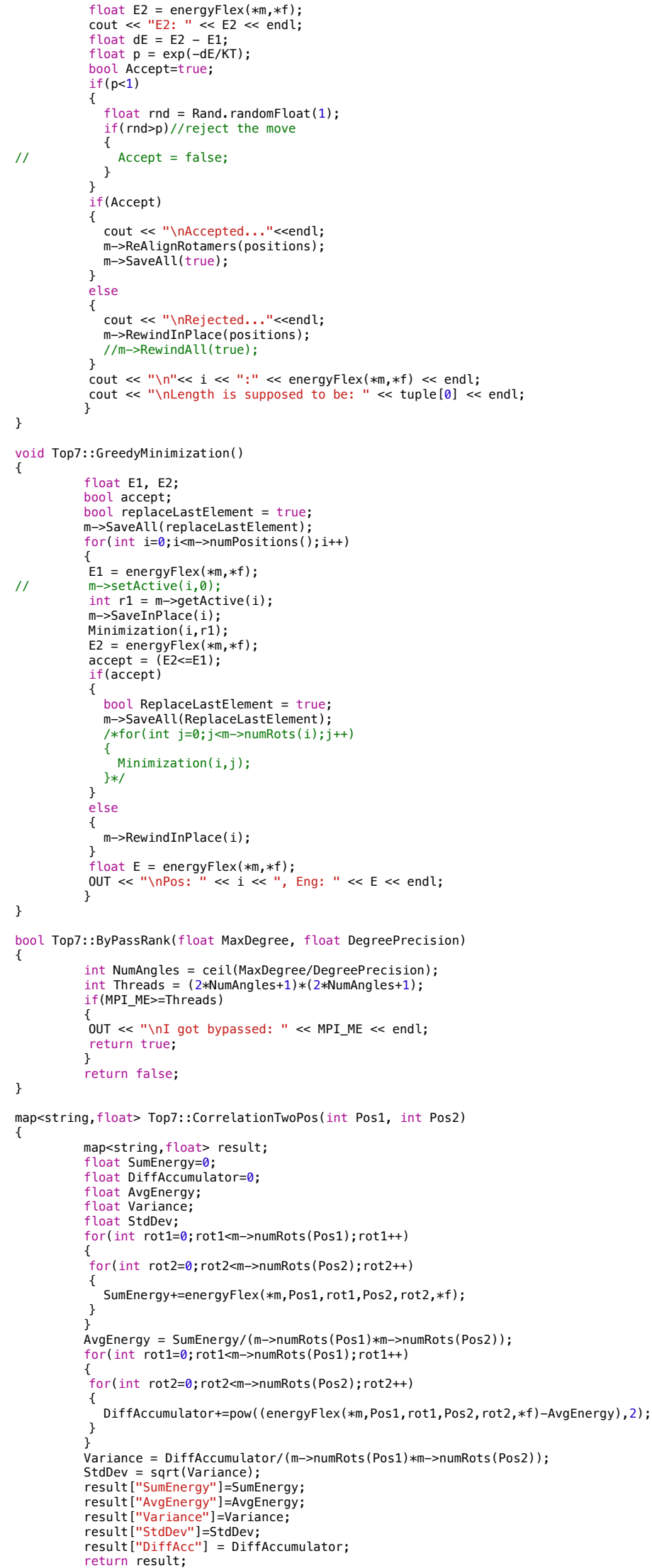


void Top7: :RotamerPerturb(int Position)

OUT $\ll$ "\n>>>Entered Top7: : Perturb() " < endl;

OUT $\ll$ "\n" $<<m->$ confkeys [Position] . size ()$<<e n d l$

int RandomRot $=$ Rand. randomInt (m->confkeys [Position]. size ( ))

OUT $\ll$ " $\backslash n "<<$ RandomRot $\ll$ endl,

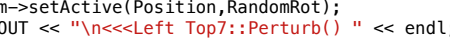

\}

void Top7: :RandomStructurePerturbation(float MaxAngle, bool Sequential)

vector<int> positions;

for (int $p=1 ; p<m->$ numPositions ()$-1 ; p++)$

\{

positions.push_back $(p)$

int Shuffles $=0$;

if (! Sequential)

for (int $i=0 ; i<$ Shuffles; $i++)$

int RandPos1 = Rand, randomInt (positions, size ())

int RandPos2 = Rand. randomInt (positions.size()),

int temp $=$ positions [RandPos2]

positions [RandPos2] = positions [RandPos1];

\}

positions [RandPos1] = temp

for (int $i=0 ; i<$ positions.size (); $i++)$

int $\mathrm{p}=$ positions [i];

float randomPhiPerturb $=$ Rand . randomf loat $(2 *$ MaxAngle $)$-MaxAngle;

float randomPsiPerturb $=$ Rand . randomFloat $(2 *$ MaxAngle $)$-MaxAngle;

float CurrentPhi $=m->\operatorname{GetPhi}(\mathrm{p})$;

$m \rightarrow$ SetPhi( $p$,CurrentPhi+randomPhiPerturb)

$m \rightarrow \operatorname{SetPsi}(\mathrm{p}$,CurrentPsi+randomPsiPerturb)

void Top7: :RamaStructurePerturbation(bool Uniform, bool Code2, float PerturbationRate)

vector $<$ int $>$ Code

vector<char> Tempcode

if (! Code2)

TempCode $=$ RamaCode0fStructure () ;

for(int $i=0 ; i<$ TempCode.size (); $i++)$

\{ Code.push_back(int (TempCode [i]));

\}

else

Code = RamaCode20fStructure ();

for ( int $\mathrm{p}=1 ; \mathrm{p}<\mathrm{m}->$ numPositions ()$-1 ; \mathrm{p++}$

int RandomNum = Rand. randomInt (1000);

if (RandomNum $>1000 *$ PerturbationRate)

continue;
OUT $\ll$ "\n Pos: " $\ll$ p $\ll$ endl

pair $<$ float, float $>$ phipsi $=$ RamaRandomPhiPsi $($ Code $[p-1]$,

$m \rightarrow$ ConfRestype (p)

(oniform,

OUT $\ll$ p $\ll "$ : " $\ll$ phipsi.first $\ll ", " \ll$ phipsi. second $\ll$ endl

float phi $=$ phipsi. first;

$m \rightarrow \operatorname{SetPhi}(p, p h i)$

$m \rightarrow \operatorname{SetPsi}(\mathrm{p}, \mathrm{psi})$;

vector<vector<int> > Top7: :GetIntMatrix(string filename)

vector<vector $<$ int $>>$ result

vector $<$ int $>$ tuple;

ifstream file(filename.c_str(), ifstream: : in);

if (!file.is_open ( ))

OUT $\ll$ "\nGetIntMatrix couldn't open its file..." $\ll$ endl;

while(file.good())

string str;

file $>$ st $r$;

if (str. compare("'")==0) break:

char $*$ pch;

vector<int> tuple

pectok( (char*)str.c_str(),","),

while(pch != NULL)

tuple.push back(atoi(pch))

result.push_back(tuple);

return result;

vector<vector<float> > Top7: :GetFloatMatrix(string filename) 


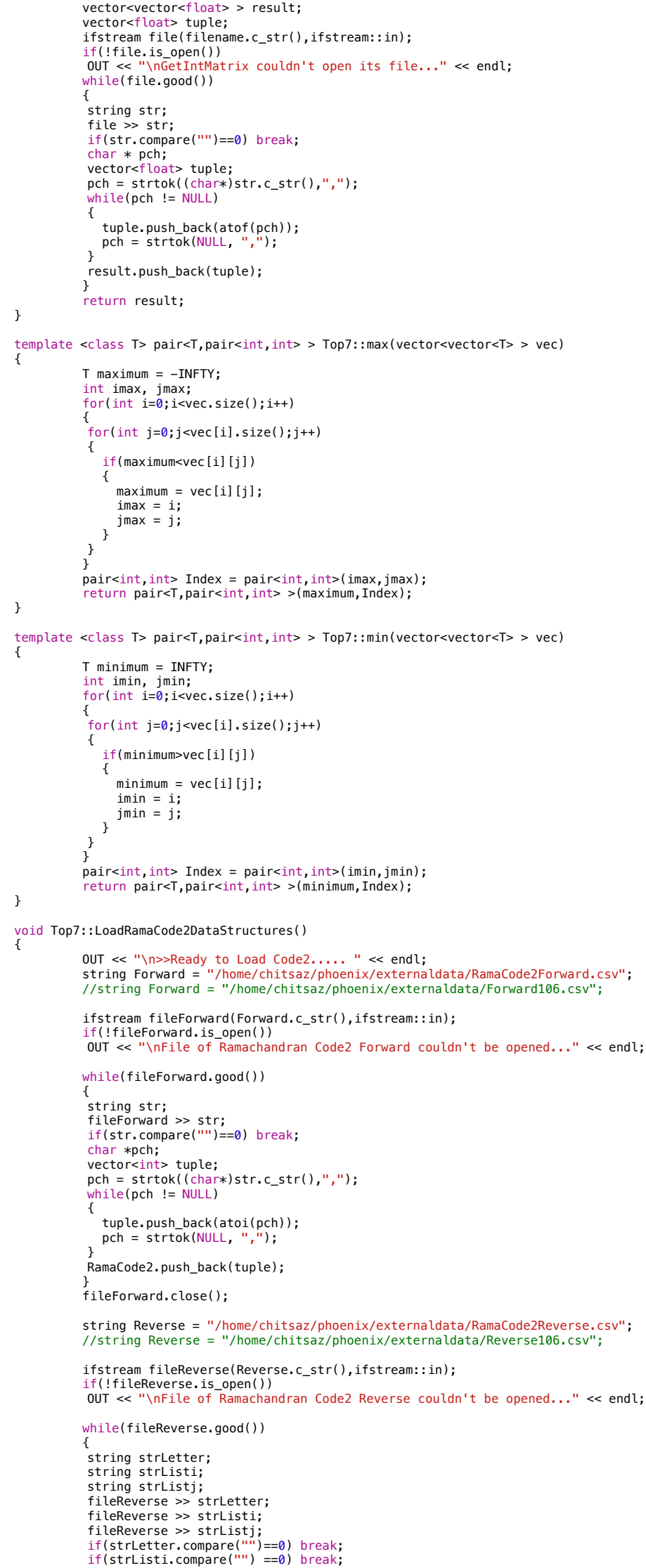




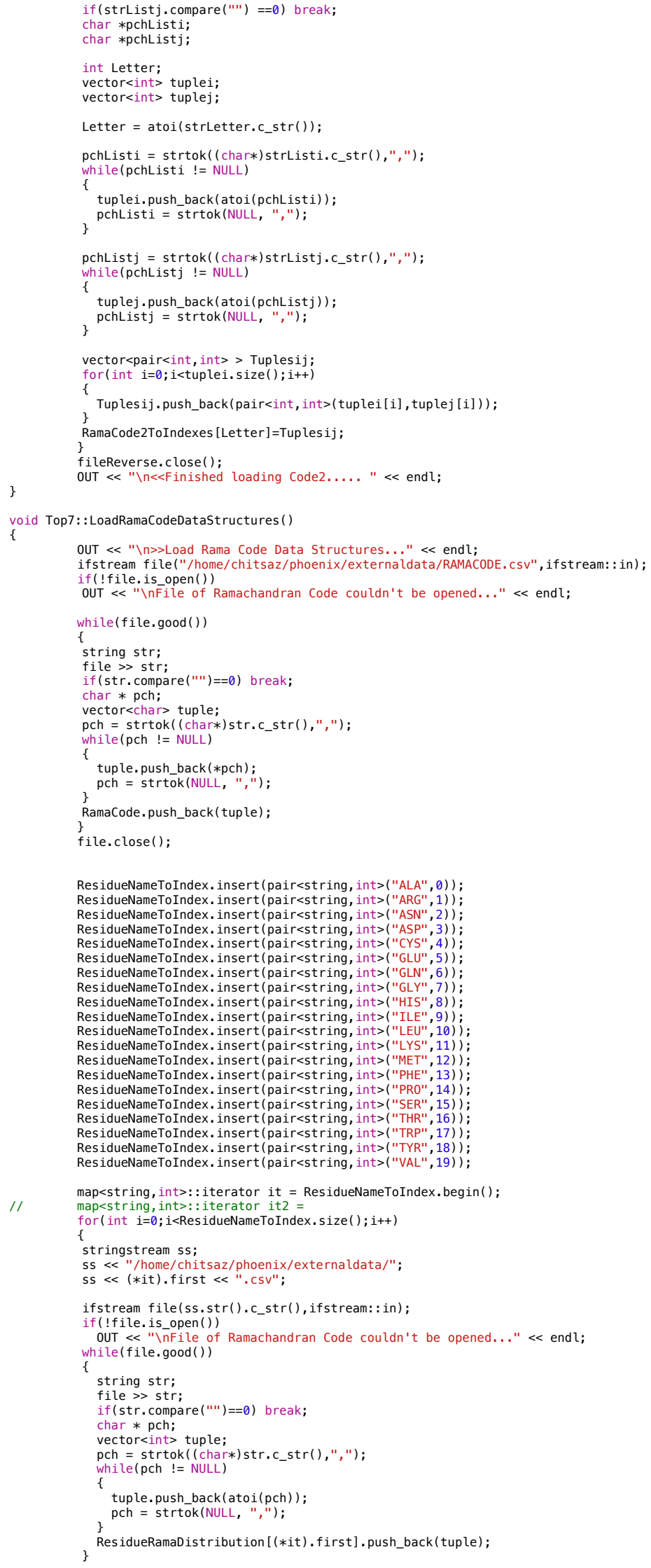


file.close();

it++

//ASCI CODE A:65, Z:90

vector<vector<pair<int, int $>>>$ temp

temp. resize(26);

for (int $i=0 ; i<36 ; i++)$

for (int $j=0 ; j<36 ; j++)$

temp [int (RamaCode [i] [j]-65) ]. push_back (pair $<$ int, int $>(i, j))$;

\}

for $($ char Code $=$ ' $A$ '; Code $<='$ ' $Z$ '; Code ++$)$

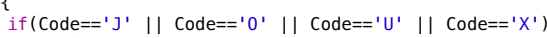

RamaCodeToIndexes [Code] $=$ temp [Code-65]:

OUT $\ll$ " $\backslash n \ll<L o a d$ Rama Code Data Structures..." $\ll$ endl;

vector $<$ int $>$ Top7: : RamaCode20fStructure()

vector<int> result

for (int $\mathrm{p}=1 ; \mathrm{p}<\mathrm{m}->$ numPositions ()$-1 ; \mathrm{p}++)$

float $\mathrm{phi}=\mathrm{m}->\operatorname{GetPhi}(\mathrm{p})+180$
float $\mathrm{psi}=\mathrm{m} \rightarrow \operatorname{GetPsi}(\mathrm{p})+180$

int $j=$ floor $(\mathrm{phi} / 10)$;

int $i=35-f l o o r(p s i / 10)$;

OUT $\ll$ "\nPOS: " $<$ p;

OUT $\ll$ "\Phi: " $<$ phi $\ll$ "\t" $\ll<j$

OUT $\ll<$ OUT $<<$ "

OUT $\ll$ " "nCode: $\ll$ RamaCode2 [i] [j] $\ll$ endl

result.push_back(RamaCode2 [i] [j]);

result;

vector<char> Top7: : RamaCode0fStructure( )

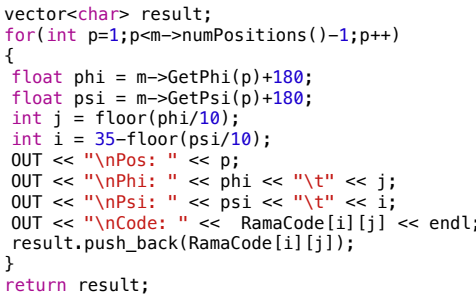

pair<float, float> Top7: : RamaRandomPhiPsi (int Code, string Residue,

boot Uniform,

OUT $\ll$ " $\backslash n "$;

OUT $\ll($ char $)$ Code $\ll "$ ", " $\ll$ Residue $\ll$ endl;

vector<pair<int, int $>>$ Indexes

if ( Code $2==$ false $)$

Indexes = RamaCodeToIndexes $[$ char (Code) $]$;

els

else

Indexes $=$ RamaCode2ToIndexes $[$ Code $]$

for (int $i=0 ; i<$ Indexes.size( $) ; i++)$

OUT $\ll "---" \ll$ Indexes [i].first $\ll ", " \ll$ Indexes [i]. second $\ll$ endl

OUT $\ll$ "\n" $\ll$ endl;

vector $<$ vector $<$ int $>>$ Distribution

if (Residue. compare ("HID") $==0$

Distribution = ResidueRamaDistribution ["HIS"]

Distribution = ResidueRamaDistribution [Residue];

for (int $i=0 ; i<$ Distribution. size();i++)

for(int $j=0 ; j<$ Distribution[i].size ()$; j++)$

OUT $\ll$ Distribution $[i][j] \ll ", "$;

OUT $\ll$ endl

*/ 


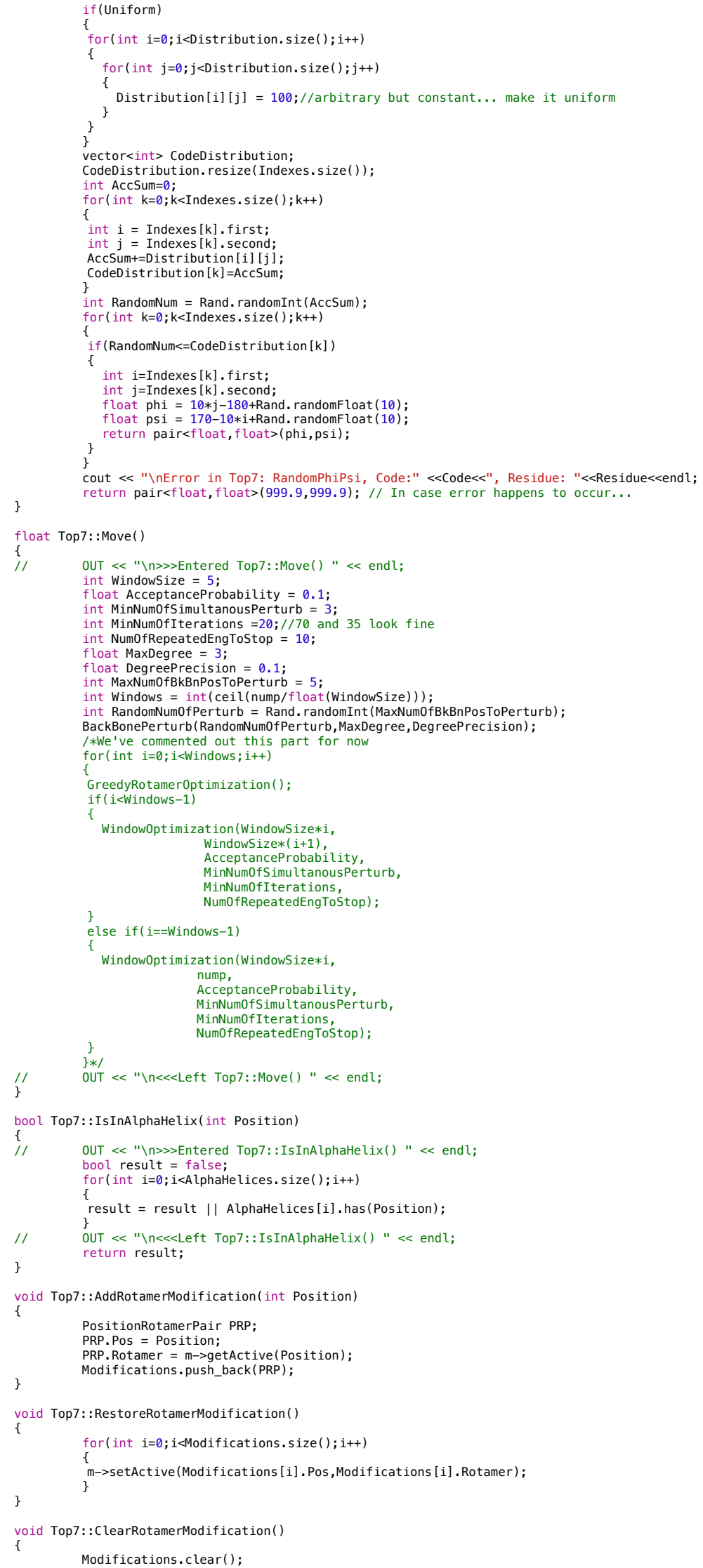

Modifications.clear(); 
void Top7: : SaveState()

RotamerConfig.clear();

for (int $i=0 ; i<$ nump; $i++$

$\{$ RotamerConfig.push_back(m->getActive(i))

CoordsConfig.clear()

for (int $i=0 ; i<m \rightarrow$ totatoms; $i++$ )

\}

CoordsConfig.push_back(m->atoms [i] $\cdot x y z)$;

template <class T> void Top7: : ShowTable (vector $<$ vector $<$ T> > vec)

for (int $i=0 ; i<\operatorname{vec} . \operatorname{size}() ; i++)$

for (int $j=0 ; j<\operatorname{vec}[i] . \operatorname{size}()-1 ; j++)$

cout $\ll \operatorname{vec}[i][j] \ll ", \backslash t "$;

cout $\ll \operatorname{vec}[i][\operatorname{vec}[i] . \operatorname{size}()-1] \ll$ endl;

cout $\ll$ "

void Top7: : RestoreState()

for (int $i=0 ; i<$ nump; $i++$ )

\{

m $\rightarrow$ setActive (i, RotamerConfig [i] );

for (int $i=0 ; i<m->$ totatoms; $i++)$

${ }_{\text {m->atoms }[i] \cdot x y z=\text { CoordsConfig }[i] \text {; }}$

Top7Error_BadPosition::Top7Error_BadPosition(string funcName, int Pos)

stringstream ss:

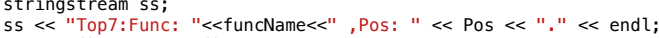
message ()+= ss. $\operatorname{str}()$;

\#ifdef BDA_PYTHON

Py0bject* MakeTop70bject(PyObject* self, Py0bject* args)

try

char* MoleculeName;

char* ForceFieldName

if ( !PyArg_ParseTuple(args, "ss", \&MoleculeName, \&ForceFieldName) return NULL;
;

new Top7 (Molecules [MoleculeName], forcefields [ForceFieldName])

OUT $\ll$ "\nWe got a new Top7 object " « endl;

stringstream name;

OfTop70bjs

" $\ll$ name. $\operatorname{str}() \ll$ endl

Numofrop70bjs++;

$\operatorname{str}()]=$ MyTop7

return Py_BuildValue("s", name.str().c_str()):

\}

catch (cpdsError\& e)

OUT $\ll$ e.Message ()$\ll$ endl;

cleanexit();

return NULL;

Py0bject* GreedyRotamerOptimization(Py0bject* self, Py0bject* args)

try

char* Top70bjName

if (!PyArg_ParseTuple(args, "s",\&Top70bjName))

return NULL;

map<string,Top7 $*>$ : : const_iterator It $=$ Top70bjects. find (Top70bjName);

if (It ! =Top70bjects. end ( )

$((* I t)$. second $) \rightarrow$ GreedyRotamerOptimization () ;

else

\}

UUT $\ll$ "\nBad Top7 0bject Name... " « endl;

catch (cpdsError\& e)

OUT $\ll$ e.Message ()$\ll$ endl;

cleanexit();

return NULL; 
$\underset{\{}{\text { Pyobject* FlexBkBn(Pyobject* self, Py0bject* args) }}$

try

char* Top70bjName;

int MaxNumOfPosToPerturb;

int Num0fIterations;

float MaxDegree;

float DegreePrecision

float ThreshHold;

float AcceptProbability;

if

PyArg_ParseTuple(args, "siiffff" ,\&Top70bjName, \&MaxNum0fPosToPerturb, \&Num0fIterations, \&MaxDegree, \&DegreePrecision, \&ThreshHold, \&AcceptProbability) ) return NULL;

map<string, Top7 $*>$ : $:$ const iterator It $=$ Top70bjects. find $($ Top70bjName $)$;

if (It!=Top70bjects. end ( )

if $((* \mathrm{It})$. second->ByPassRank(MaxDegree, DegreePrecision $))$ return Py_BuildValue( $(s "$ ", "PassedBy_done");
$(* \mathrm{It})$. second $\rightarrow$ FlexBkBn (MaxNumOfPosToPerturb,

MaxDegree,

DegreePrecision,

else

AcceptProbability):

$\{$ OUT $\ll$ "\nBad Top7 Object Name...$" \ll$ endl;

return Py_BuildValue("s", "done");

catch (cpdsError\& e)

OUT $\ll$ e.Message $($ ) $\ll$ endl;

cleanexit();

return NULL;

PyObject* RossetaMove(Py0bject* self, Py0bject* args)

try

char* Top70bjName

int NumPos;

int Steps:

float MaxDegree

float PrecisionDegree

float KTi;

if(!PyArg_ParseTuple(args, "siiffff", \&Top70bjName,

\&NumPos,
\&Steps,

\&steps,

\&PrecisionDegree

QKTf) ) return NULL,

map<string, Top7 $*>$ : : const_iterator $I t=$ Top70bjects. $f$ ind $($ Top70bjName $)$;

if (It !=Top70bjects. end ()$)$

$(* I t)$. second->RossetaMove (NumPos,

Steps,

PrecisionDegree,

KTi,

els

\{

$\ll$ "InBad Top70bj Name ...." " endl;

return Py_BuildValue("s", "done")

catch (cpdsError\& e)

OUT $\ll$ e.Message ()$\ll$ endl;

cleanexit();

Py0bject* FlexBkBnAnnealing(Py0bject* self, Pyobject* args)

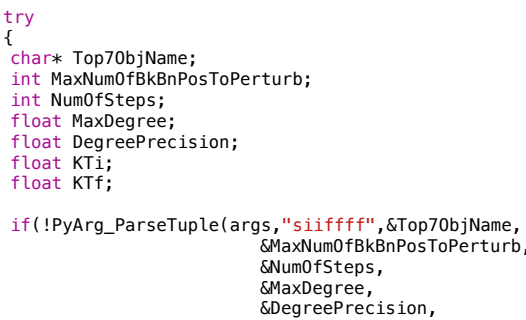


\&KTi,

map<string,Top7 $*>$ : : const iterator It $=$ Top70bjects. find $($ Top70bjName $)$ if (It !=Top70bjects.end ( ))

if $((* \mathrm{It})$. second->ByPassRank (MaxDegree, DegreePrecision)) return Py_BuildValue("s", "PassedBy_done"); $(* I t)$. second $\rightarrow$ FlexBkBnAnnealing (MaxNum0 fBkBnPosToPerturb, Num0fSteps,

MaxDegree,

DegreePrecision,

els

KTf);

\{

OUT $\ll$ "\nBad Top70bj Name .... " $\ll$ endl;

return Py_BuildValue("s", "done"),

catch (cpdsError\& e)

OUT $\ll$ e.Message ()$\ll$ endl;

cleanexit( );

return NULL;

Py0bject* FlexBackrubMonteCarlo(Pyobject* self, Py0bject* args)

try

char* Top70bjName

float MaxAngle;

float Precision

float KTi

float KTf:

int Steps;

int Length $=3$;

float BiasProbability $=0.5$;

bool domin=false;

if(!PyArg_ParseTuple(args, "sffffi|ifi" ,\&Top70bjName, sMaxAngle,

\&Preci
\&KTi,
\&KTf,

\&KTf,

\&Steps,

\&BiasProbability

\&domin)) return NULL:

map<string,Top7 $*>$ : : const_iterator It $=$ Top70bjects. find (Top70bjName); if (It! =Top70bjects. end ())

$(* \mathrm{It})$. second->FlexBackrubMonteCarlo(MaxAngle,

Precision,

KTf,

Steps,

BiasProbability

els

domin):

else

OUT $\ll$ "\nBad Top70bj Name ...." $\ll$ endl;

return Py_BuildValue("s", "done")

catch (cpdsError\& e)

OUT $\ll$ e.Message ()$\ll$ endl

cleanexit( );

return NULL;

Py0bject* Flex0strichMonteCarlo(Pyobject* self, Py0bject* args)

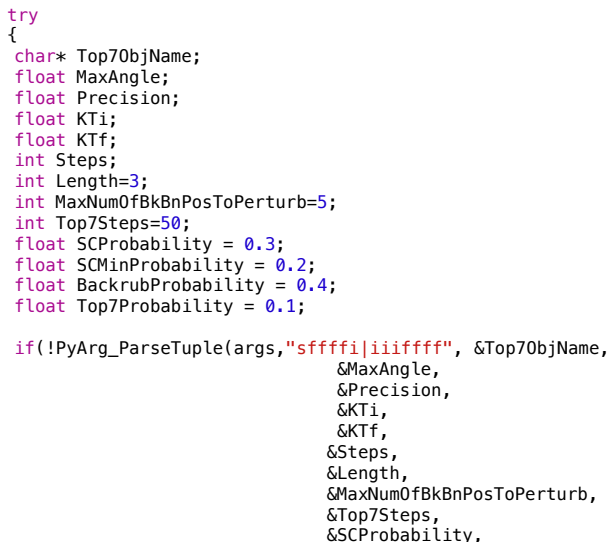




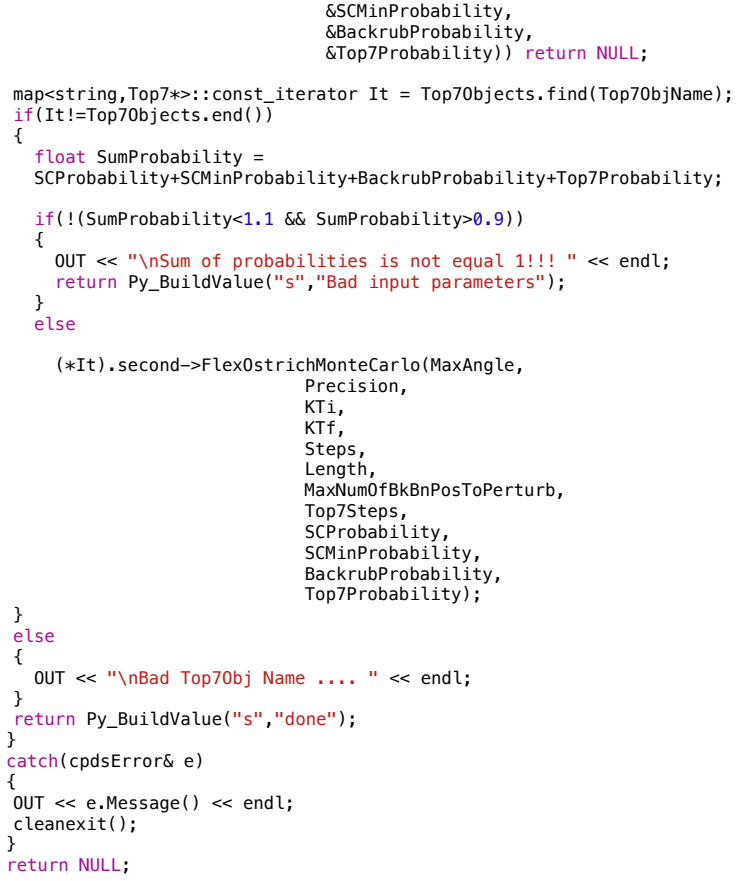

Py0bject* DisruptStructure(Py0bject* self, Pyobject* args)

$$
\text { try }
$$

char* Top70bjName

Char* Top70bjName;
int MaxNumOfPosToPerturb;

int Num0fIterations;

float MaxDegree;

float DegreePrecision:

float EnergyStepIncrease

float ThreshHold;

float AcceptProbability:

(!PyArg_ParseTuple (args, "siifffff" ,\&Top70bjName, \&MaxNumOfPosToPerturb, \&Num0fIterations, \&MaxDegree, \&DegreePrecision, \&EnergyStepIncrease, \&ThreshHold, \&AcceptProbability))
return NULL;

map<string, Top7 $*>$ : : const_iterator $I t=$ Top70bjects. find $($ Top70bjName $)$;

if (It! =Top70bjects. end ()

\{

if $((* \mathrm{It})$. second->ByPassRank (MaxDegree, DegreePrecision))

return Py_BuildValue("s", "PassedBy_done");

$(* \mathrm{It})$. second $\rightarrow$ Dis ruptSt ructure (MaxNumOfPosToPerturb

Num0fIterations,

MaxDegree,

Degreecision,

Threshtopld

els

AcceptProbability):

els

T $\ll$ "\Bad Top70bj Name.... " $\ll$ endl

return Py_BuildValue("s", "done")

catch (cpdsError\& e)

OUT $\ll$ e.Message ( ) $\ll$ endl;

cleanexit( );

return NULL;

Pyobject* ShuffleBkBn (Pyobject* self, Pyobject* args)

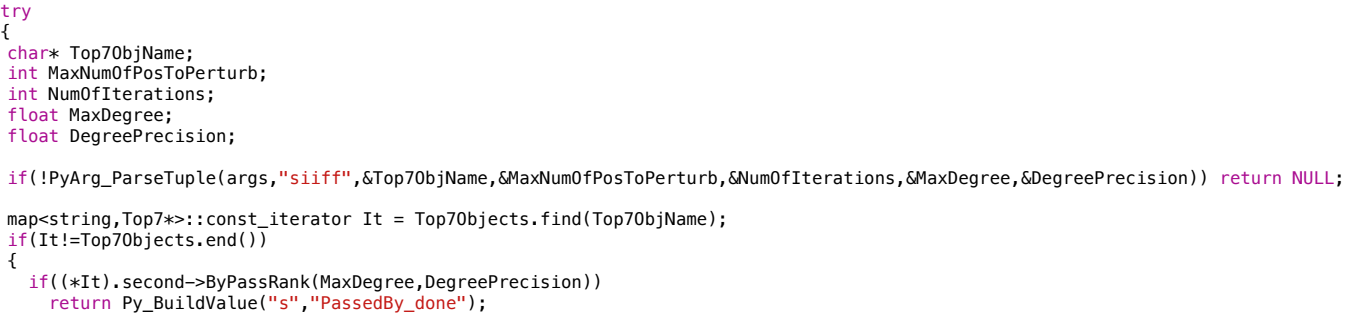




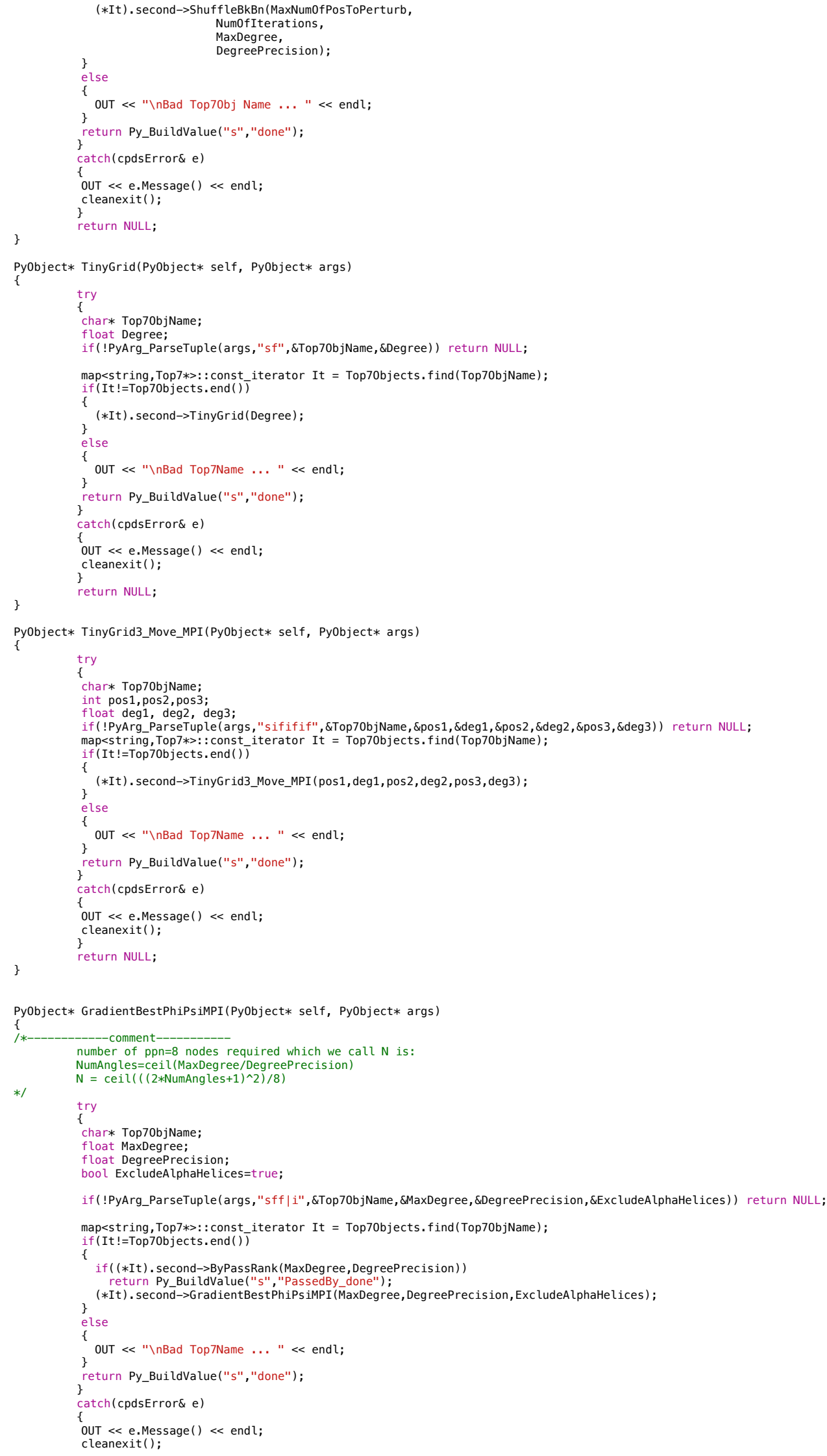



return NULL;

Py0bject* GradientBestPhiPsi(Py0bject* self, Py0bject* args)

\{ number of ppn $=8$ nodes required which we call $\mathrm{N}$ is: NumAngles=ceil (MaxDegree/DegreePrecision) $\mathrm{N}=\operatorname{ceil}(((2 *$ NumAngles +1$) \wedge 2) / 8)$

$* /$

try

char* Top70bjName;

float MaxDegree;

float DegreePrecision;

bool Rotameroptimization=true

aphots $=$ false

if ( !PyArg_ParseTuple (args, "sff|ii" ,\&Top70bjName, \&MaxDegree, \&DegreePrecision, \&RotamerOptimization, \&RecordSnapShots)) return NULL;

map<string, Top7 $*>$ : : const iterator It $=$ Top70bjects. find(Top70bjName);

if ( It ! =Top70bjects. end ( ))

// if $((* \mathrm{It})$. second->ByPassRank(MaxDegree, DegreePrecision)) \} $(* I t)$. second $\rightarrow$ GradientBestPhiPsi (MaxDegree, DegreePrecision, RotamerOptimization, RecordSnapShots); els

OUT $\ll$ "\nBad Top7Name ... " $\ll$ endl

return Py_BuildValue("s", "done");

catch (cpdsError\& e)

OUT $\ll$ e.Message ()$\ll$ endl;

cleanexit();

\}

Py0bject* GradientBestPhiPsiMPI_HighPerformance(Pyobject* self, Py0bject* args)

number of $\mathrm{ppn}=8$ nodes required which we call $\mathrm{N}$ is:

greePrecision)

*/

try

char* Top70bjName

int StartPos;

int StopPos;

float MaxDegree;

float DegreePrecision:

bool ExcludeAlphaHelices=false

bool RecordSnapShots=false;

NULL;

if ( !PyArg_ParseTuple(args, "siiff|ii",\&Top70bjName, \&StartPos, \&StopPos, \&MaxDegree, \&DegreePrecision, \&ExcludeAlphaHelices, \&RecordSnapShots) ) return

map<string, Top7 $*>$ : : const_iterator $I t=$ Top70bjects. find $($ Top70bjName) ;

if (It!=Top70bjects.end ( ) )

StartPos--;

StopPos--:

(F) . second $\rightarrow$ ByPassRank (MaxDegree, DegreePrecision))

(return Py_BuildValue("s", "PassedBy_done"); else

OUT $\ll$ "\nBad Top7Name ... " $\ll$ endl;

return Py_BuildValue("s", "done");

\} catch (cpdsError\& e)

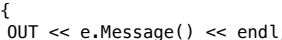

cleanexit();

return NULL;

Py0bject* GradientBestPhiPsiBackrubMPI_HighPerformance(Pyobject* self, PyObject* args)

\{

number of $\mathrm{ppn}=8$ nodes required which we call $\mathrm{N}$ is:

NumAngles=ceil (MaxDegree/DegreePrecision)

*/

$\mathrm{N}=\operatorname{ceil}(((2 *$ NumAngles +1$) \wedge 2) / 8$

try

char* Top70bjName;

int StartPos:

int StopPos;
float MaxDegree;

float DegreePrecision;

bool ExcludeAlphaHelices=false

int BackrubLength=3;

float BackrubDegree $=4.0$;

float BackrubPrecision $=0.02$;

if ( !PyArg_ParseTuple(args, "siiff |iiff" ,\&Top70bjName, \&StartPos , \&StopPos, \&MaxDegree, \&DegreePrecision, \&ExcludeAlphaHelices , \&BackrubLength , \&Backrub-

Degree, \&BackrubPrecision)) return NULL; 


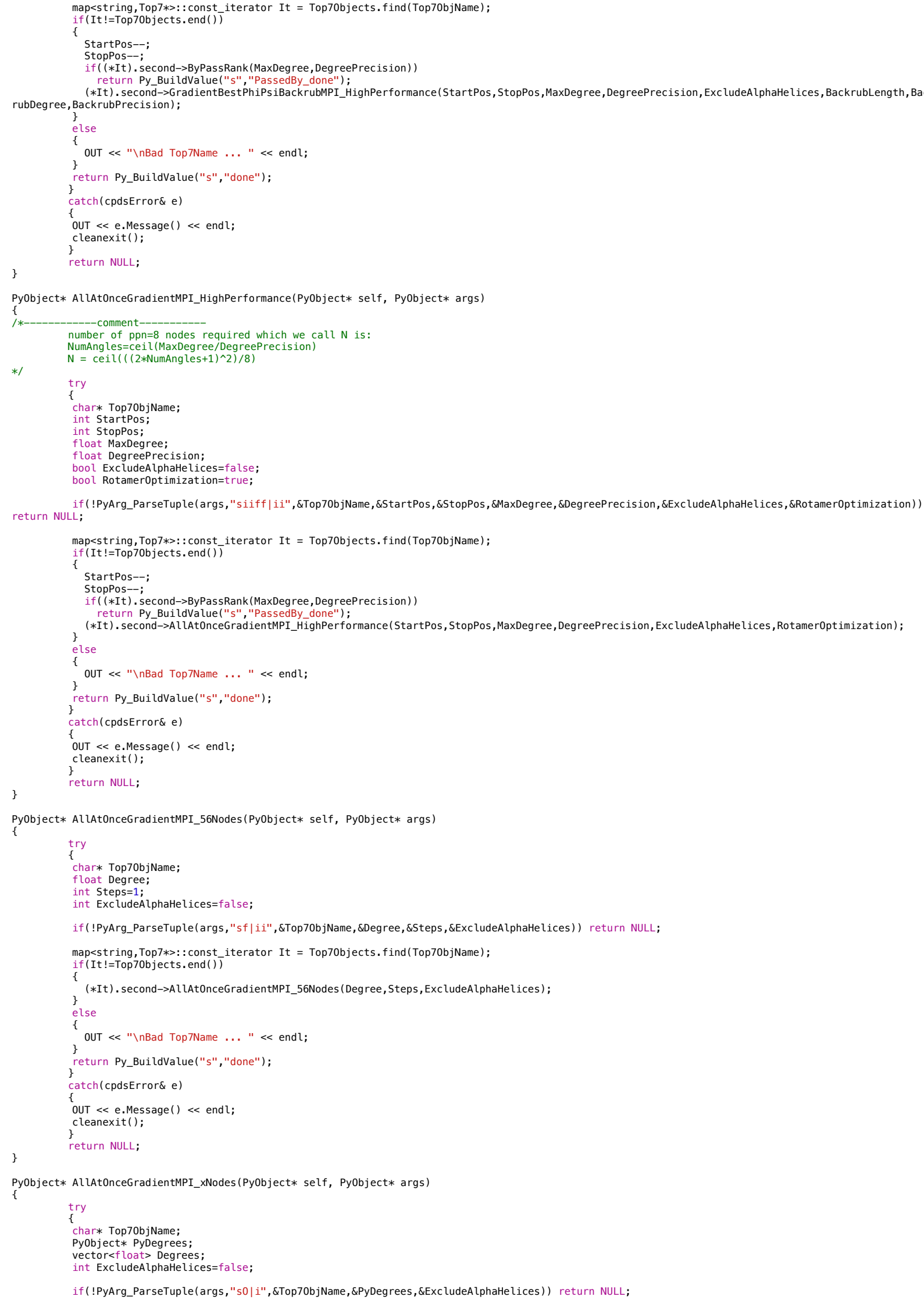




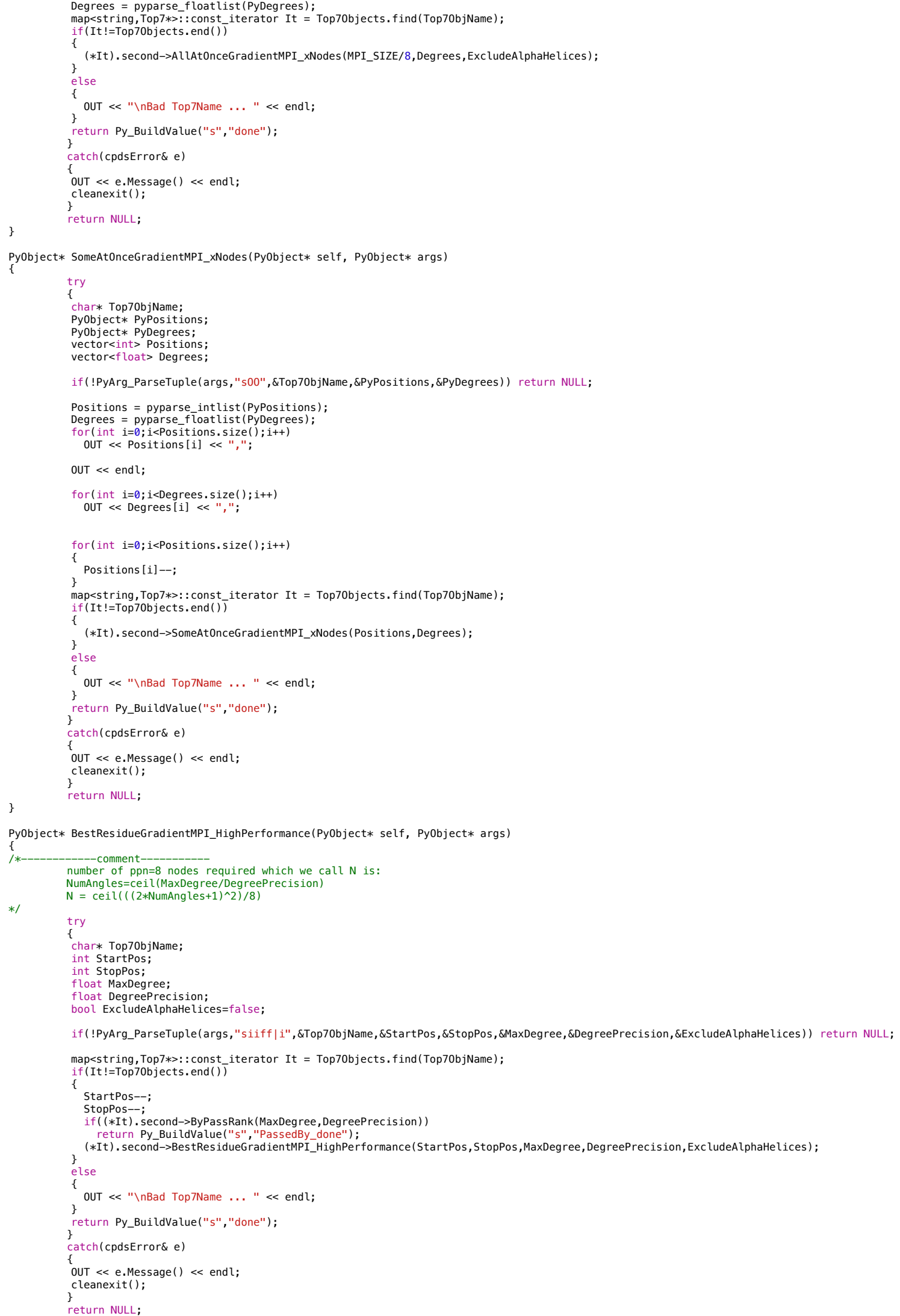




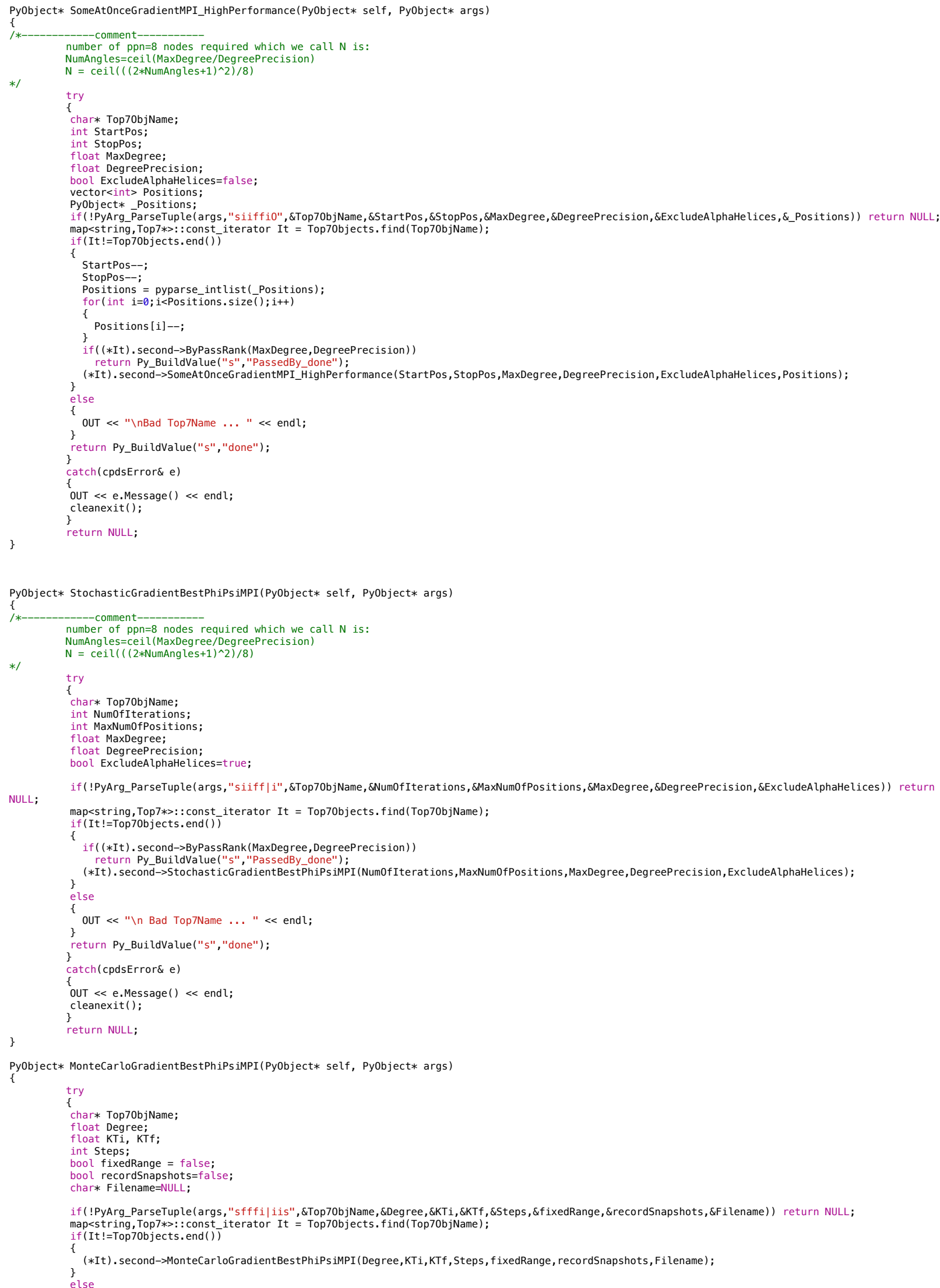




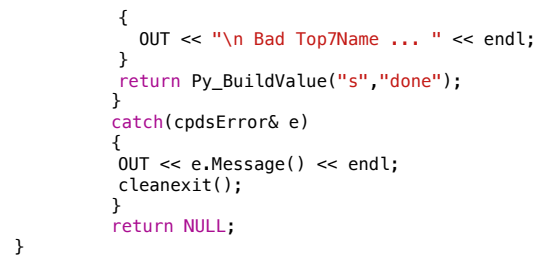

Py0bject* StochasticPerturbationGridRefinement(Py0bject* self, Py0bject* args)

try

char* Top70bjName;

Py0bject* PyDegrees;

int Num0fStructs;

float MaxPerturbationAngle;

char* path;

char* name;

int UseRamaCode $=1$;

int UniformRama $=0$;

int RecordSnapShots $=$ false

bool Code2=false;

bool doGRID = true;

vector<float> degrees;

vector $<$ float> precision;

if(!PyArg_ParseTuple(args, "s00ifssiiiii|i", \&Top70bjName,

\&PyDegrees,

\&PyPrecision,

\&Num0fStructs,

\&MaxPerturbationAngle,

\&path,

\&name,

\&UseRamaCode,

\&UniformRama,

\&Code2

\&doGRID) ) return NULL

degrees $=$ pyparse_floatlist $($ PyDegrees $)$ :

precision = pyparse_floatlist(PyPrecision):

OUT $\ll$ "\ndegrees: " $<$ endl,

for (int $i=0 ; i<\operatorname{deg} r e e s . \operatorname{size}() ; i++)$

OUT $\ll$ degrees $[i] \ll<", "$;

for (int $i=0 ; i<$ precision. $\operatorname{size}() ; i++$

OUT $\ll$ precision [i] $\ll ", "$;

map<string,Top7 $*>$ : : const_iterator It $=$ Top70bjects. find $($ Top70bjName $)$; if (It! =Top70bjects.end ( ) )

$\{(* \mathrm{It})$. second->StochasticPerturbationGridRefinement (degrees,

precision,

Num0fStructs

MaxPerturbationAngle,

path,

name,

UseRamaCode,

UniformRama,

RecordSnapShots,

Code2,

else

OUT $\ll " \backslash n$ Bad Top7Name ..." $" \ll$ endl.

return Py_BuildValue("s", "done");

catch (cpdsError\& e)

OUT $\ll$ e.Message $($ ) $\ll$ endl;

cleanexit( );

\}

Py0bject* StochasticPerturbationBackrubRefinement(Py0bject* self, Py0bject* args)

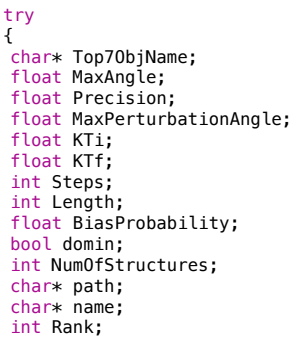


bool RecordSnapShots;

if(! PyArg_ParseTuple(args, "sfffffiifiissii",

\&Top70bjName,

sMaxAngle,

\&Precision,

\&KTi,

\&KTf,

\&Steps,

\&Length,

\&BiasProbability,

\&domin,

NumofStructures,

\&path,

\&name,

(RecordSnapShots)) return NULL;

map<string, Top7 $*>$ : : const iterator It $=$ Top70bjects. find (Top70bjName) ;

map<st ring, Top7 $*>$ : : const
if (It ! $=$ Top70bjects. end ()$)$

( $* \mathrm{It}$ ). second $\rightarrow$ StochasticPerturbationBackrubRef inement (MaxAngle,

$$
\text { Precision, }
$$

MaxPerturbationAngle,

KTi,

KTf,

Steps,

BiasProbability,

domin,

Num0fS' ructures,

path,

name,

Rank,

$\stackrel{\text { else }}{\{}$

RecordSnapShots);

OUT $\ll$ "\n Bad Top7Name ... " $\ll$ endl;

return Py_BuildValue("s", "done")

catch (cpdsError\& e)

OUT $\ll$ e.Message ()$\ll$ endl;

cleanexit():

return NULL;

Py0bject* StochasticRamaPerturbationDecoyGeneration (Pyobject* self, Pyobject* args)

$\operatorname{tr}$

char* Top70bjName;

int Number0fStructures

char* path;
char* name;

float PerturbationRate $=0.1$

if(!PyArg_ParseTuple(args, "sissf",

Top70bjName,

sNumberOfStructures,

\&path,

\&PerturbationRate)) return NULL

map<string,Top7 $*>$ : : const_iterator $I t=$ Top70bjects. find (Top70bjName) ;

if (It!=Top70bjects.end ()$)$

$(* I t)$. second $\rightarrow$ StochasticRamaPerturbationDecoyGeneration (Number0fStructures, path,

else

PerturbationRate);

OUT $\ll$ "\n Bad Top7Name ... " $\ll$ endl;

return Py_BuildValue("s", "done")

catch (cpdsError\& e)

OUT $\ll$ e.Message () $\ll$ endl;

cleanexit ();

Py0bject* ReplicaExchangeMonteCarloGRID(Py0bject* self, Py0bject* args)

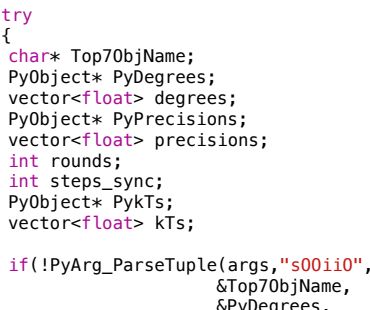




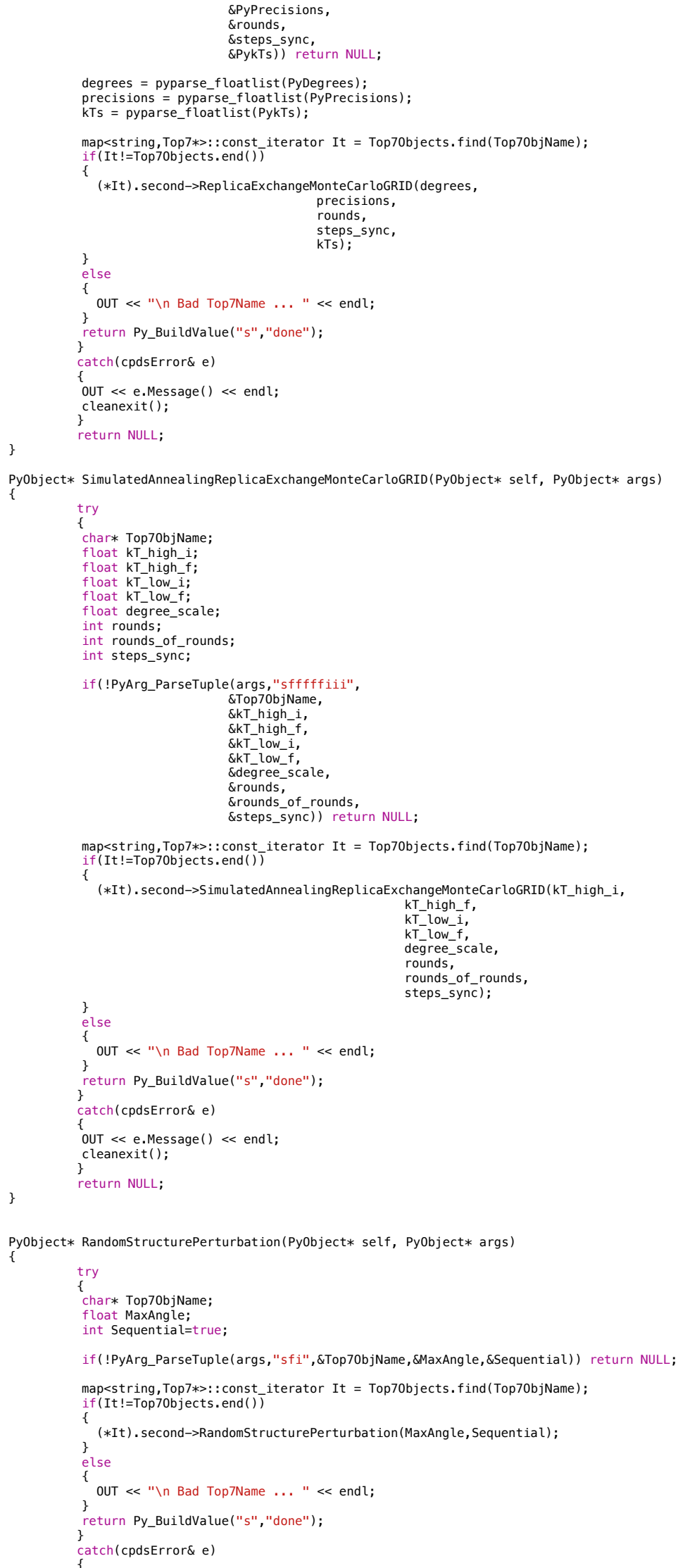

Py0bject* RandomStructurePerturbation (Pyobject* self, Pyobject* args) 


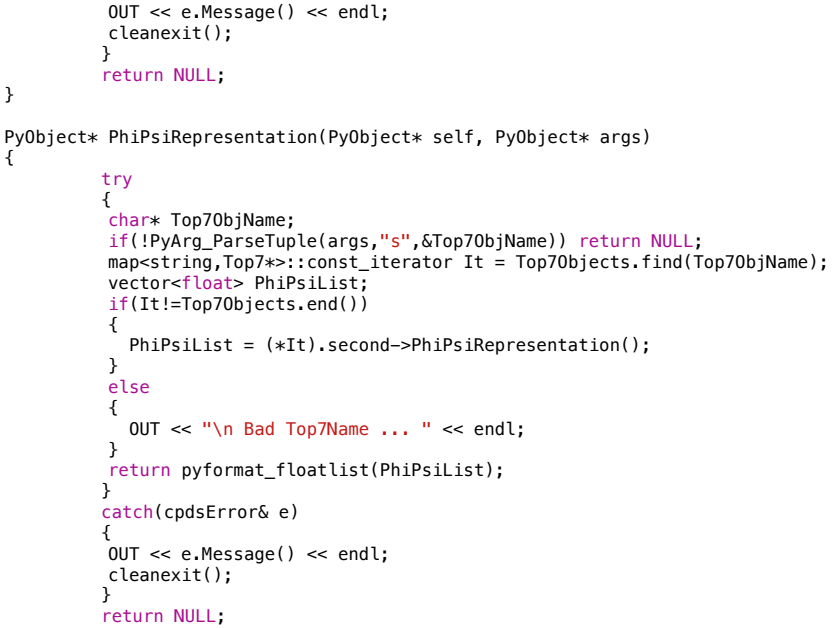

Py0bject* GreedyMinimization(Py0bject* self, Py0bject* args)

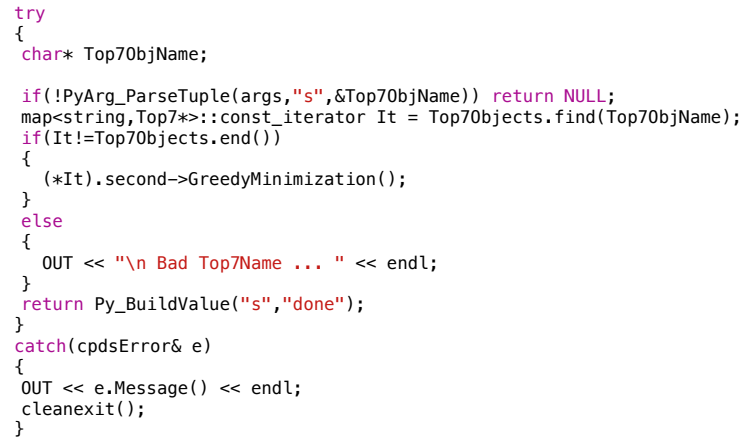


return NULL;

Py0bject* AlignTwoMoleculesRMSD (Pyobject* self, Py0bject* args)

/this manipulates coordinates of the second molecule...

try

char* MoleculeName1;

if (!PyArg_ParseTuple (args, "ss", \&MoleculeName1, \&MoleculeName2)) return NULL;

molecules* M1 = get_molecules (MoleculeName1)

molecules* M2 = get_molecules (MoleculeName2);

vector $<$ keyrange $>k 1=M 1 \rightarrow$ getActivesAll () ;

vector $<$ keyrange $>\mathrm{k} 2=$ M2 $>$ getActivesAll ()

float rmsdValue $=$ alignAndRMSD (M1->atoms, M2->atoms, k1, k2)

return Py_BuildValue("f", rmsdValue);

atch (cpdsError\& e)

OUT $\ll$ e.Message ()$\ll$ end ,

cleanexit();

return NULL;

\#endif 